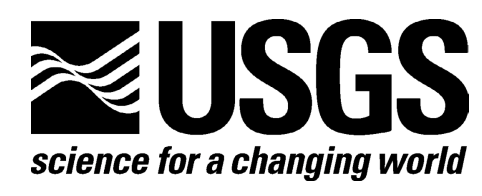

\title{
Argon dating at and near Medicine Lake volcano, California: Results and data
}

By Julie M. Donnelly-Nolan and Marvin A. Lanphere

2005

Open-File Report 2005-1416

U.S. Department of the Interior U.S. Geological Survey 


\section{U.S. Department of the Interior Gale A. Norton, Secretary \\ U.S. Geological Survey \\ P. Patrick Leahy, Acting Director}

U.S. Geological Survey, Reston, Virginia 2005

Revised and reprinted: 2005

For product and ordering information:

World Wide Web: http://www.usgs.gov/pubprod

Telephone: 1-888-ASK-USGS

For more information on the USGS - the Federal source for science about the Earth, its natural and living resources, natural hazards, and the environment:

World Wide Web: http://www.usgs.gov

Telephone: 1-888-ASK-USGS

Any use of trade, product, or firm names is for descriptive purposes only and does not imply endorsement by the U.S. Government.

Although this report is in the public domain, permission must be secured from the individual copyright owners to reproduce any copyrighted material contained within this report. 


\section{Contents}

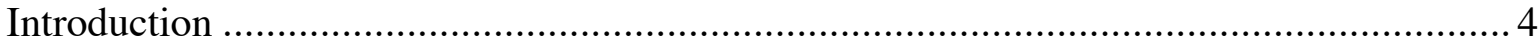

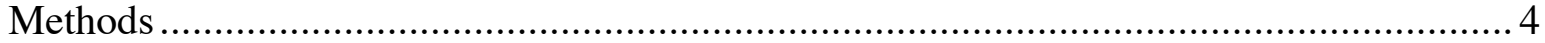

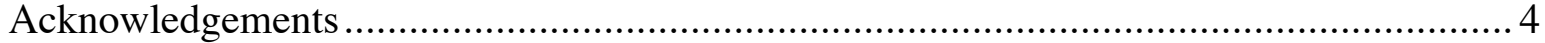

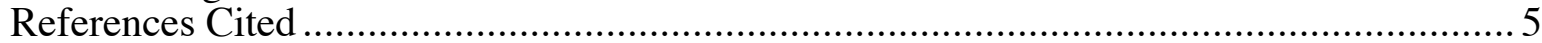

\section{Figures}

1. Location map showing approximate outline of Medicine Lake volcano lavas, which are shown in gray. Dots are locations of argon-dated samples with sample numbers shown only for samples collected outside the area of Figure 2. The + symbols indicate locations of drill holes; hole identifiers are shown adjacent to symbol. LBNM is Lava Beds National Monument .......................................................... 7

2. Enlarged location map showing argon-dated samples not labeled on Figure 1. ............ 8

\section{Tables}

1. Potassium-Argon ages, Medicine Lake volcano and vicinity.

2. ${ }^{40} \mathrm{Ar} /{ }^{39} \mathrm{Ar}$ ages, Medicine Lake volcano and vicinity .......................................... 13

3. Data for ${ }^{40} \mathrm{Ar} /{ }^{39} \mathrm{Ar}$ ages measured at USGS, Menlo Park ....................................... 15

4. Data for ${ }^{40} \mathrm{Ar} /{ }^{39} \mathrm{Ar}$ ages measured at Berkeley Geochronology Center ....................... 29

5. Comparison of early rhyolite ages, Medicine Lake volcano .................................... 37 


\section{INTRODUCTION}

This report presents $\mathrm{K}-\mathrm{Ar}$ and ${ }^{40} \mathrm{Ar} /{ }^{39} \mathrm{Ar}$ ages and supplementary data measured in support of geologic mapping at Medicine Lake volcano, northern California. Interpretation of the significance of these ages to be presented elsewhere (DonnellyNolan, in preparation). Sample locations are shown in Figures 1 and 2. Previous publications describing argon dating at and near the volcano include Brown and Mertzman (1979), Luedke and Lanphere (1980), Mertzman (1977, 1981, 1982, 1983), McKee and others (1983), Becker and others (1994), Herrero-Bervera and others, 1994, Donnelly-Nolan and others (1994, 1996), and Turrin (1996).

\section{METHODS}

All of the ages listed in Table 1 are conventional K-Ar ages measured in the USGS Menlo Park laboratory on whole-rock samples selected after thin-section examination. Decay constants (Steiger and Jager, 1977) are listed in Table 1. $\mathrm{K}_{2} \mathrm{O}$ measurements were made by flame photometry after lithium metaborate fusion and dissolution (Ingamells, 1970). Ar analyses were by isotope-dilution mass spectrometry using a high-purity (>99.9\%) ${ }^{38} \mathrm{Ar}$ tracer and techniques described previously (Dalrymple and Lanphere, 1969). All samples for Ar extraction were baked overnight at $280^{\circ} \mathrm{C}$. Mass analyses were done on a $22.68 \mathrm{~cm}$ radius, multiple-collector mass spectrometer with a nominal $90^{\circ}$ sector magnet, using automated data collection (Stacey and others, 1981; Sherrill and Dalrymple, 1980).

${ }^{40} \mathrm{Ar} /{ }^{39} \mathrm{Ar}$ incremental-heating experiments in the Menlo Park laboratory (Table 2) were made on splits of approximately $100 \mathrm{mg}$ of sample material. The resistanceheated furnace used to extract $\mathrm{Ar}$ is attached to the cleanup system and mass spectrometer described by Dalrymple (1989). The furnace is modified from the design of Staudacher and others (1978). Heating temperatures were controlled with an optical fiber thermometer. The fluence monitor for USGS ${ }^{40} \mathrm{Ar} /{ }^{39} \mathrm{Ar}$ analyses was 856003 sanidine, a secondary mineral standard with a reference age of $27.92 \mathrm{Ma}$.

In an incremental-heating experiment, the sample is heated to a given temperature and an apparent age is calculated for the gas extracted at that temperature. In calculating an apparent age, it is assumed that the non-radiogenic Ar in a sample is atmospheric in isotopic composition. Analytical data for the USGS determinations are given in Table 3.

${ }^{40} \mathrm{Ar} /{ }^{39} \mathrm{Ar}$ ages measured at the Berkeley Geochronology Center (Table 2) used the techniques described in Herrero-Bervera and others (1994) and in Turrin (1996). Available analytical data are given in Table 4.

One additional table is included (Table 5) which compares ages determined on early rhyolite units of the volcano, both for this project and with previous work. 


\section{ACKNOWLEDGEMENTS}

Funding for this work was provided by the USGS Geothermal and Volcano Hazards Programs. We thank B. Turrin for personal communications containing some of the data presented here and for discussions of the data. Turrin's work was terminated by the USGS Reorganization in 1995. L. B. Gray generated many of the K-Ar analyses, organized the data, and calculated final ages for other determinations. We are grateful for the laboratory assistance of J. Saburomaru, F. McFarland, J. Markman, G. Elliott, M. Olea, and others at the USGS geochronology facility in Menlo Park CA. R. Luedke collected sample 78C4, D. Adam collected sample CBL, and J. Smith collected the CSJ series of pre-MLV samples. Drill hole samples were collected from stored core at the Energy and Geoscience Institute in Salt Lake City, Utah. We are also grateful to the geothermal energy companies exploring at Medicine Lake volcano for making the samples available.

\section{REFERENCES CITED}

Becker, T.A., Sharp, W.D., Renne, P.R., Turrin, B.D., Page, W.D., and Wakabayashi, J., $1994,{ }^{40} \mathrm{Ar} /{ }^{39} \mathrm{Ar}$ dating of young low-K tholeiites: examples from northeast California, U.S.A.: Abstracts of the Eighth International Conference on Geochronology, Cosmochronology, and Isotope Geology, U.S. Geological Survey Circular 1107, p.

Brown, Laurie, and Mertzman, S. A., 1979, Negative inclination anomalies from the Medicine Lake Highland lavas, northern California: Earth and Planetary Science Letters, v. 42, p. 121-126.

Dalrymple, G.B., 1989, The GLM continuous laser system for ${ }^{40} \mathrm{Ar} /{ }^{39} \mathrm{Ar}$ dating: Description and performance characteristics: U.S. Geological Survey Bulletin 1890, p. 89-96.

Dalrymple, G.B., and Lanphere, M.A., 1969, Potassium-argon dating: San Francisco, W.H. Freeman and Co., 258 p.

Donnelly-Nolan, J.M., in preparation, Geologic map of Medicine Lake volcano, scale 1:50,000: USGS map submitted

Donnelly-Nolan, J.M., Smith, J.G., Champion, D.E., and Lanphere, M. A., 1996, A Pleistocene back-arc basalt center, northeastern CA: Geological Society of America Abstracts with Programs, v. 28, no. 5, p. 62.

Donnelly-Nolan, J.M., Turrin, B.D., Gray, L.B., and Conrad, J.E., 1994, Incomplete extraction of radiogenic argon from high-silica andesites: implications for K-Ar dating: Abstracts of the Eighth International Conference on Geochronology, Cosmochronology, and Isotope Geology, U.S. Geological Survey Circular 1107, p. 84.

Herrero-Bervera, E., Helsley, C.E., Sarna-Wojcicki, A.M., Lajoie, K.R., Meyer, C.E., McWilliams, M.O., Negrini, R.M., Turrin, B.D., Donnelly-Nolan, J.M., and Liddicoat, J.C., 1994, Age and correlation of a paleomagnetic episode in the western United States by ${ }^{40} \mathrm{Ar} /{ }^{39} \mathrm{Ar}$ dating and tephrochronology: The Jamaica, Blake, or a new polarity episode?: Journal of Geophysical Research, v. 99, p. 24,091-24,103. 
Ingamells, C.O., 1970, Lithium metaborate flux in silicate analysis: Analytica Chimica Acta, v. 52, p. 323-334.

Luedke, R.G., and Lanphere, M.A., 1980, K-Ar ages of Upper Cenozoic volcanic rocks, northern California: Isochron/West, no. 28, p. 7-8.

McKee, E.H., Duffield, W.A., and Stern, R.J., 1983, Late Miocene and early Pliocene basaltic rocks and their implications for crustal structure, northeastern California and south-central Oregon: Geological Society of America Bulletin, v. 94, p. 292304.

Mertzman, S.A., 1977, The petrology and geochemistry of the Medicine Lake Volcano, California: Contributions to Mineralogy and Petrology, v. 62, p. 221-247.

Mertzman, S.A., 1981, Pre-Holocene silicic volcanism on the northern and western margins of the Medicine Lake Highland, California: USGS Circular 838, p. 163169.

Mertzman, S.A., 1982, K-Ar results for silicic volcanics from the Medicine Lake Highland, northeastern California - a summary: Isochron/West, no. 34, p. 3-7.

Mertzman, S.A., 1983, An addendum to "K-Ar results for silicic volcanics from the Medicine Lake Highland, northeastern California - a summary", Isochron/West, no. 38, p. 3-5.

Sherrill, N.D., and Dalrymple, G.B., 1980, A computerized multi-channel data acquisition and control system for high-precision mass spectrometry: U.S. Geological Survey Professional paper 1129-A, p. A1-A6.

Stacey, J.S., Sherrill, N.D., Dalrymple, G.B., Lanphere, M.A., and Carpenter, N.V., 1981, A five-collector system for the simultaneous measurement of argon isotopic ratios in a static mass spectrometer: International Journal of Mass Spectrometry \& Ion Physics, v. 39, p. 167-180.

Staudacher, T., Jessberger, E.K., Dörflinger, D., and Kiki, J., 1978, A refined ultrahighvacuum furnace for rare gas analysis: Journal Physics E: Scientific Instruments, v. 11, p. 781-784.

Steiger, R.H., and Jager, E., 1977, IUGS Subcommission on Geochronology: convention on the use of decay constants in geo- and cosmochronology: Earth and Planetary Science Letters, v. 36, p. 359-362.

Turrin, B.D., 1996, Development and application of ${ }^{40} \mathrm{Ar} /{ }^{39} \mathrm{Ar}$ laser-fusion dating and ${ }^{40} \mathrm{Ar} /{ }^{39} \mathrm{Ar}$ step-heating dating of Quaternary basaltic volcanic rocks: a comparison of conventional K-Ar dating and ${ }^{40} \mathrm{Ar} /{ }^{39} \mathrm{Ar}$ dating methods: $\mathrm{Ph}$.D. Dissertation, University of California, Berkeley CA. 


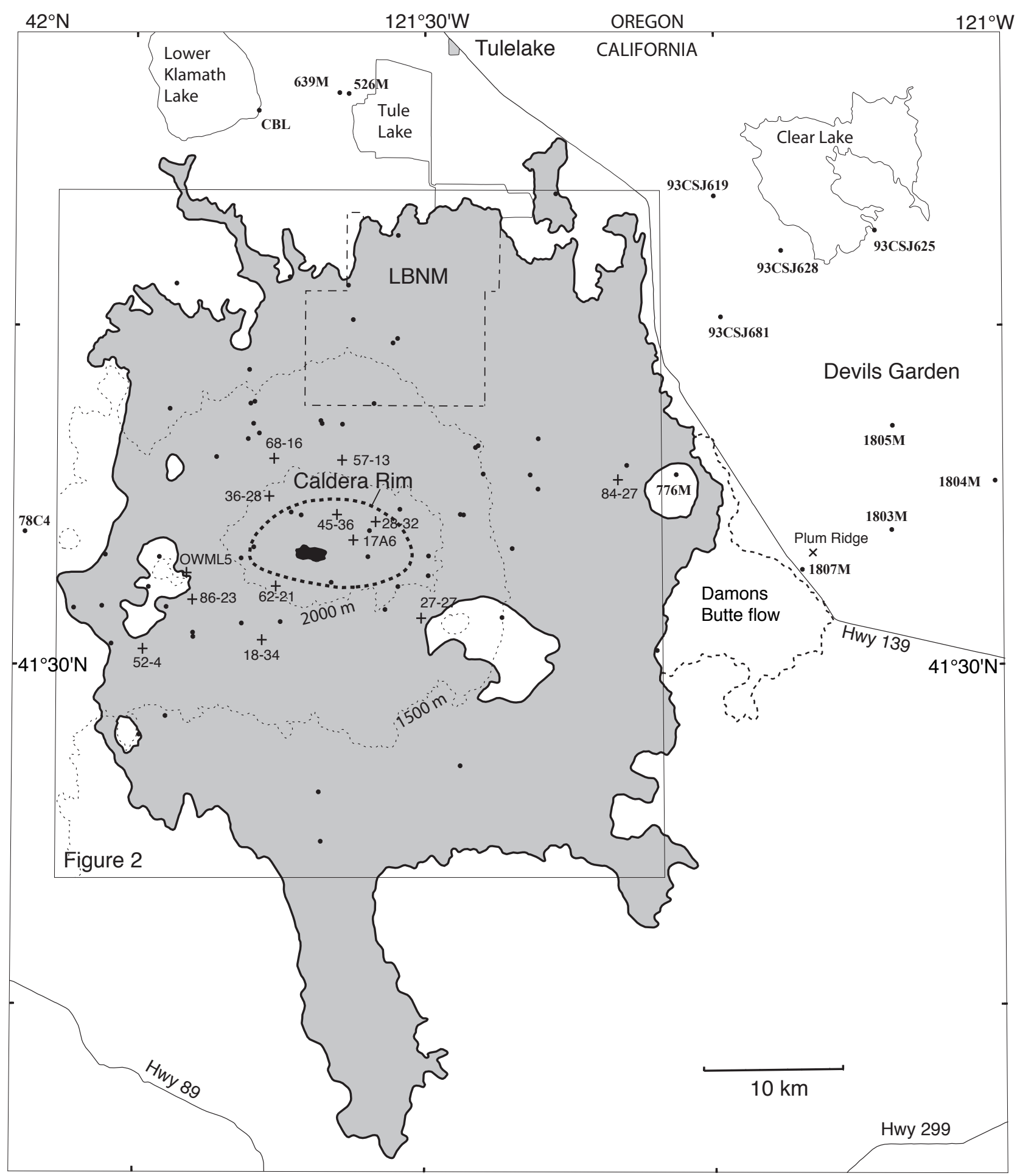

Figure 1. Location map showing approximate outline of Medicine Lake volcano lavas, which are shown in gray. Dots are locations of argon-dated samples with sample numbers shown only for rocks collected outside the area of Figure 2. The + symbols indicate locations of drill holes; hole identifiers are shown adjacent to symbol. LBNM is Lava Beds National Monument. 


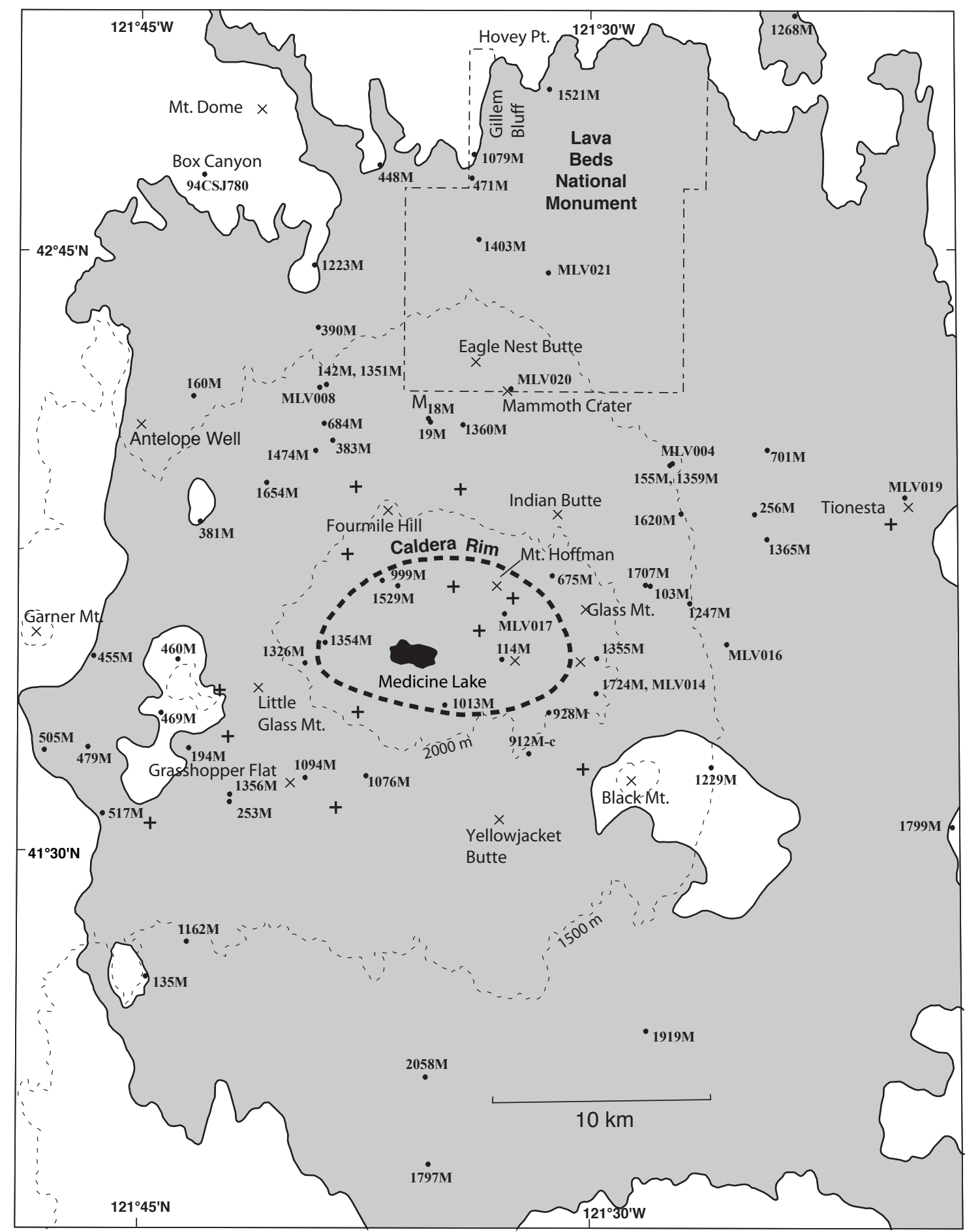

Figure 2. Enlarged location map showing argon-dated samples not labeled on Figure 1. 
Table 1. Potassium-Argon Ages, Medicine Lake volcano and vicinity

All samples are whole-rock; all ages in ka (thousands of years) except as noted

Decay constant: ${ }^{\star} \lambda_{\varepsilon}=0.581 \times 10^{-10} \mathrm{yr}^{-1} ; \lambda_{\beta}=4.962 \times 10^{-10} \mathrm{yr}^{-1} ;{ }^{40} \mathrm{~K} / \mathrm{K}=1.167 \times 10^{-4} \mathrm{~mol} / \mathrm{mol}$

\begin{tabular}{|c|c|c|c|c|c|c|c|c|c|c|c|c|}
\hline Sample \# & $\#$ Description & Unit & Average age & Ages & $\begin{array}{l}\text { Wt. \% } \mathrm{K}_{2} \mathrm{O} \\
\text { used }\end{array}$ & $\begin{array}{c}\mathrm{K}_{2} \mathrm{O} ' \mathrm{~s} \\
\mathrm{n}=\end{array}$ & $\begin{array}{l}\text { Weight } \\
\text { (gms) }\end{array}$ & $\begin{array}{c}\begin{array}{c}40 \\
\mathrm{Ar} \\
(\mathrm{mole} / \mathrm{g})\end{array} \\
\end{array}$ & $\begin{array}{c}\%^{40} \mathrm{Ar} \\
\mathrm{rad}\end{array}$ & Other information & $\begin{array}{l}\text { Lat. } \\
41^{\circ} \mathrm{N} \\
\end{array}$ & $\begin{array}{l}\text { Long. } \\
121^{\circ} \mathrm{W} \\
\end{array}$ \\
\hline & MLV ages & & & & & & & & & & Min. & Min. \\
\hline \multirow[t]{3}{*}{$18 \mathrm{M}$} & Rhyolite E. of Callahan Flow, & rec & $313 \pm 22$ & $284 \pm 32$ & $4.342 \pm 0.059$ & 4 & 4.607 & $1.775 \times 10^{-12}$ & 19.6 & same unit as $19 \mathrm{M}$ & 40.81 & 35.38 \\
\hline & aphyric (obsidian) & & & $293 \pm 30$ & & & 4.7961 & $1.834 \times 10^{-12}$ & 22 & & & \\
\hline & & & & $326 \pm 16$ & & & 7.3543 & $2.39 \times 10^{-12}$ & 35.7 & & & \\
\hline \multirow[t]{3}{*}{$19 M$} & $\begin{array}{l}\text { Rhyolite E. of Callahan Flow, } \\
\text { aphyric (dull obsidian) }\end{array}$ & rec & $335 \pm 21$ & $\begin{array}{l}309 \pm 18 \\
393 \pm 27\end{array}$ & $4.280 \pm 0.144$ & 2 & $\begin{array}{l}6.9776 \\
5.4488\end{array}$ & $\begin{array}{l}1.902 \times 10^{-12} \\
2.422 \times 10^{-12}\end{array}$ & $\begin{array}{l}18.9 \\
11.6\end{array}$ & same unit as $18 \mathrm{M}$ & 40.77 & 35.37 \\
\hline & $\begin{array}{l}\text { weighted avg, } 18 \mathrm{M}+19 \mathrm{M} \\
\text { weighted avg.18M,19M,68-16- }\end{array}$ & & $322 \pm 22$ & & & & & & & & & \\
\hline & 753 & & $301 \pm 10$ & & & & & & & & & \\
\hline \multirow[t]{2}{*}{$103 \mathrm{M}$} & Rhyolite E. of Glass Mountain & reg & $707 \pm 28$ & $607 \pm 44$ & $4.735 \pm 0.106$ & 2 & 5.026 & $4.139 \times 10^{-12}$ & 24.7 & $0.48 \pm 0.06 \mathrm{Ma}$ (Mertzman, 1983, & 36.68 & 28.15 \\
\hline & (obsidian) & & & $732 \pm 22$ & & & 4.6327 & $4.993 \times 10^{-12}$ & 22.2 & $\begin{array}{l}\text { no. 16); see Table 2, no.26: } \\
{ }^{40} \mathrm{Ar} /{ }^{39} \mathrm{Ar} \text { age }\end{array}$ & & \\
\hline \multirow[t]{3}{*}{$142 \mathrm{M}$} & Lower rhyolite W. of Callahan & $\mathrm{rgf}$ & $486 \pm 20$ & $486 \pm 20$ & $4.548 \pm 0.059$ & 4 & 4.8562 & $3.189 \times 10^{-12}$ & 21.3 & $0.61 \pm 0.03 \mathrm{Ma}$ (Mertzman, 1982, & 41.68 & 38.90 \\
\hline & Grasshopper Flat (obsidian) & & & & & & & & & & & \\
\hline & weighted avg. $\mathrm{w} / 1351 \mathrm{M}$ & & $371 \pm 13$ & & & & & & & & & \\
\hline \multirow[t]{2}{*}{$155 \mathrm{M}$} & Rhyolite near Cougar Butte & $\mathrm{rcb}$ & $590 \pm 22$ & $574 \pm 21$ & $4.407 \pm 0.025$ & 3 & 6.6118 & $3.645 \times 10^{-12}$ & 44.7 & $0.43 \pm 0.04 \mathrm{Ma}$ (Mertzman, 1982, & 39.66 & 27.40 \\
\hline & $\begin{array}{l}\text { (obsidian) } \\
\text { weighted avg.w/ 1359M }\end{array}$ & & $569 \pm 19$ & $609 \pm 23$ & & & 5.8732 & $3.863 \times 10^{-12}$ & 50.6 & $\begin{array}{l}\text { no. } 11 \text { ); same unit as } 1359 \mathrm{M} \text {; see } \\
\text { Table 2, no. } 35:{ }^{40} \mathrm{Ar} /{ }^{39} \mathrm{Ar} \text { age }\end{array}$ & & \\
\hline \multirow[t]{6}{*}{$253 M$} & Rhyolite of Grasshopper Flat & rgf & $445 \pm 31$ & $486 \pm 43$ & $4.640 \pm 0.030$ & 3 & 3.2236 & $3.243 \times 10^{-12}$ & 8.3 & $0.33 \pm 0.02 \mathrm{Ma}$ (Mertzman, 1982, & 31.24 & 42.00 \\
\hline & (obsidian) & & & $430 \pm 26$ & & & 5.3337 & $2.877 \times 10^{-12}$ & 8.4 & no.12; same unit as $1356 \mathrm{M}$ & & \\
\hline & second sample & & $272 \pm 6$ & $263 \pm 8$ & $4.670 \pm 0.002$ & 3 & 15.843 & $1.765 \times 10^{-12}$ & 32.8 & & & \\
\hline & & & & $284 \pm 9$ & & & 11.66 & $1.913 \times 10^{-12}$ & 42.8 & & & \\
\hline & weighted avg. of 4 determinations & & $284 \pm 12$ & & & & & & & & & \\
\hline & weighted avg. $\mathrm{w} / 1356 \mathrm{M}$ & & $276 \pm 11$ & & & & & & & & & \\
\hline \multirow[t]{2}{*}{$256 \mathrm{M}$} & $\begin{array}{l}\text { Rhyolite S. of Little Sand Butte } \\
\text { (obsidian) }\end{array}$ & $\mathrm{rsl}$ & $327 \pm 16$ & $\begin{array}{l}364 \pm 18 \\
302+15\end{array}$ & $4.700 \pm 0.024$ & 2 & 5.1722 & $2.467 \times 10^{-12}$ & 10.1 & $0.24 \pm 0.03 \mathrm{Ma}$ (Mertzman, 1982, & 38.38 & 24.71 \\
\hline & weighted avg. w/ 1365M & & $313 \pm 11$ & & & & & & & & & \\
\hline \multirow[t]{3}{*}{$505 \mathrm{M}$} & Andesite of Typhoon Mesa & atm & $254 \pm 25$ & $284 \pm 44$ & $1.705 \pm 0.010$ & 3 & 17.4948 & $6.979 \times 10^{-13}$ & 2.5 & & 32.48 & 48.19 \\
\hline & & & & $242 \pm 49$ & & & 19.24 & $5.951 \times 10^{-13}$ & 2 & & & \\
\hline & & & & $241 \pm 35$ & & & 15.5504 & $5.915 \times 10^{-13}$ & 2.7 & & & \\
\hline \multirow[t]{2}{*}{$517 \mathrm{M}$} & Dacite E. of Lost Spring & dls & $182 \pm 4$ & $171 \pm 6$ & $3.403 \pm 0.015$ & 3 & 20.338 & $8.387 \times 10^{-13}$ & 24.1 & $0.05 \pm 0.045 \mathrm{Ma}$ (Mertzman, & 30.95 & 46.23 \\
\hline & & & & $192 \pm 6$ & & & 20.012 & $9.443 \times 10^{-13}$ & 15.1 & 1982, no.1) & & \\
\hline \multirow[t]{2}{*}{$675 \mathrm{M}$} & Rhyolite N.W. of Glass Mountain & rng & $105 \pm 3$ & $109 \pm 4$ & $4.473 \pm 0.031$ & 3 & 22.138 & $7.013 \times 10^{-13}$ & 20.8 & $0.05 \pm 0.01 \mathrm{Ma}$ (Mertzman, 1983, & 36.97 & 31.40 \\
\hline & & & & $99 \pm 5$ & & & 19.464 & $6.347 \times 10^{-13}$ & 16.8 & no.18) & & \\
\hline \multirow[t]{2}{*}{$684 \mathrm{M}$} & Rhyolite W. of Callahan Flow & rwc & $349 \pm 7$ & $339 \pm 10$ & $4.647 \pm 0.032$ & 3 & 9.964 & $2.270 \times 10^{-12}$ & 26 & & 40.69 & 38.90 \\
\hline & (upper); (obsidian) & & & $360 \pm 11$ & & & 12.529 & $2.412 \times 10^{-12}$ & 33.7 & & & \\
\hline \multirow[t]{3}{*}{ 999M } & Andesite of the north rim & anr & $78 \pm 6$ & $74 \pm 12$ & $1.721 \pm 0.018$ & 3 & 19.851 & $1.841 \times 10^{-13}$ & 5.4 & Table 2, no. 15: ${ }^{40} \mathrm{Ar} /{ }^{39} \mathrm{Ar}$ age & 36.78 & 37.05 \\
\hline & & & & $78 \pm 8$ & & & 32.383 & $1.924 \times 10^{-13}$ & 6.3 & \& Donnelly-Nolan \& others, 1994 & & \\
\hline & & & & $83 \pm 11$ & & & 17.17 & $2.067 \times 10^{-13}$ & 4.1 & & & \\
\hline
\end{tabular}




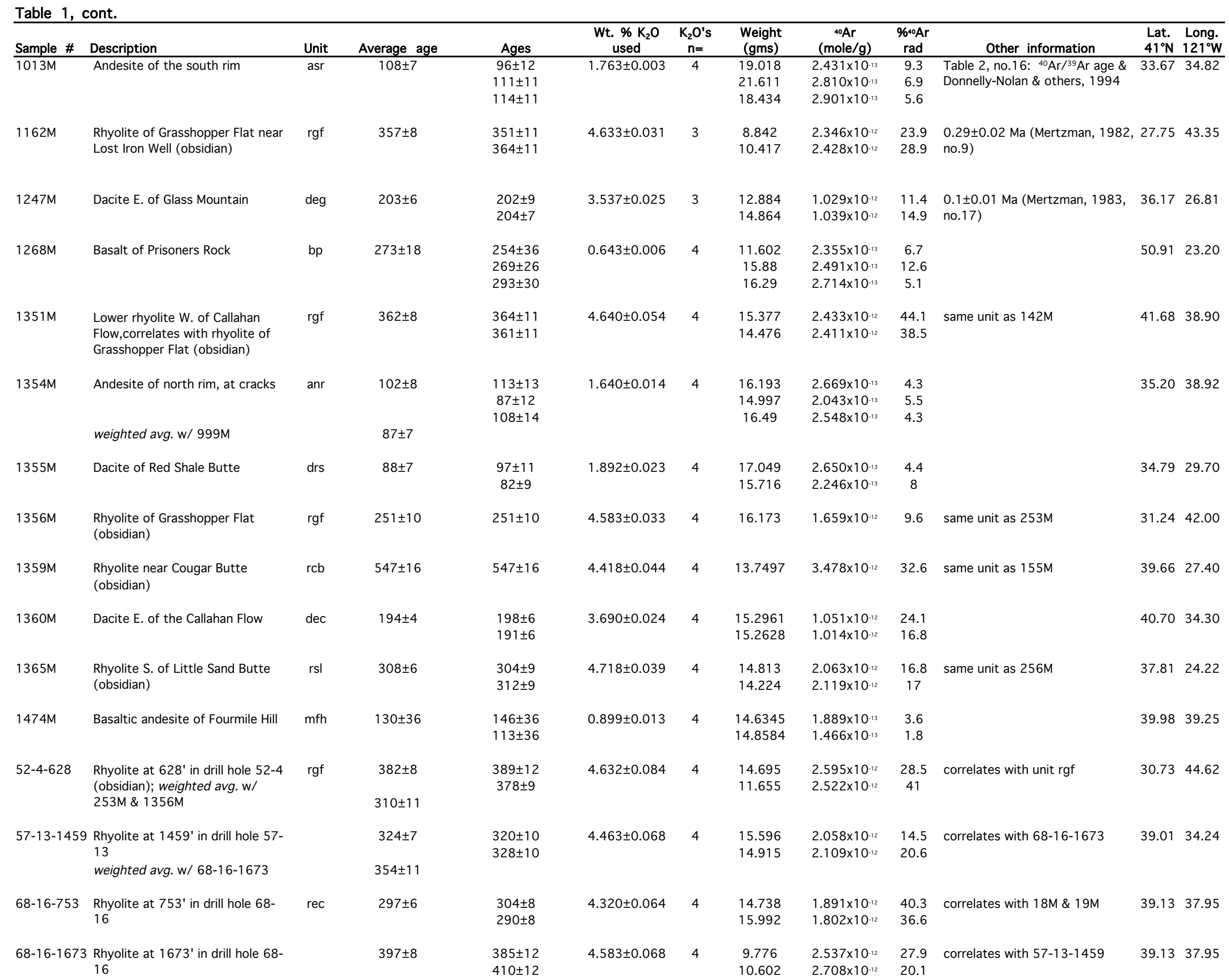


Table 1, cont.

\begin{tabular}{|c|c|c|c|c|c|c|c|c|c|c|c|c|}
\hline Sample \# & Description & Unit & Average age & Ages & $\begin{array}{c}\text { Wt. } \% \mathrm{~K}_{2} \mathrm{O} \\
\text { used }\end{array}$ & $\begin{array}{c}\mathrm{K}_{2} \mathrm{O} \text { 's } \\
\mathrm{n}=\end{array}$ & $\begin{array}{l}\text { Weight } \\
\text { (gms) }\end{array}$ & $\begin{array}{c}{ }^{40} \mathrm{Ar} \\
(\mathrm{mole} / \mathrm{g}) \\
\end{array}$ & $\begin{array}{l}\%^{* 0} \mathrm{Ar} \\
\mathrm{rad}\end{array}$ & Other information & $\begin{array}{l}\text { Lat. } \\
41^{\circ} \mathrm{N} \\
\end{array}$ & $\begin{array}{l}\text { Long. } \\
121^{\circ} \mathrm{W}\end{array}$ \\
\hline $135 \mathrm{M}$ & $\begin{array}{l}\text { Pre-Medicine Lake volcano } \\
\text { Dacite of Harris Mt. }\end{array}$ & $\begin{array}{l}\text { ages } \\
\text { odh }\end{array}$ & $\begin{array}{l}\text { (or not } M L V \text { ) } \\
2.95 \pm 0.09 \mathrm{Ma}\end{array}$ & $\begin{array}{l}2.96 \pm 0.09 \mathrm{Ma} \\
2.93 \pm 0.09 \mathrm{Ma}\end{array}$ & $2.023 \pm 0.020$ & 5 & $\begin{array}{l}3.0941 \\
6.5465\end{array}$ & $\begin{array}{l}8.635 \times 10^{-12} \\
8.550 \times 10^{-12}\end{array}$ & $\begin{array}{l}30.4 \\
29.8\end{array}$ & & 26.90 & 44.67 \\
\hline \multirow[t]{2}{*}{$381 \mathrm{M}$} & $\begin{array}{l}\text { Older rhyolite of Dock Well (W. } \\
\text { dome) }\end{array}$ & ord & $959 \pm 73$ & $959 \pm 73$ & $3.830 \pm 0.019$ & 1 & 2.4127 & $5.289 \times 10^{-12}$ & 5.2 & & 38.20 & 43.07 \\
\hline & second sample & & $823 \pm 28$ & $\begin{array}{l}826 \pm 49 \\
814 \pm 46\end{array}$ & $3.847 \pm 0.031$ & 3 & $\begin{array}{l}8.954 \\
8.492\end{array}$ & $\begin{array}{l}4.574 \times 10^{-12} \\
4.511 \times 10^{-12}\end{array}$ & $\begin{array}{c}6.6 \\
7\end{array}$ & $\begin{array}{l}0.95 \pm 0.14 \mathrm{Ma} \text { (Mertzman, 1982, } \\
\text { no.5) }\end{array}$ & & \\
\hline $448 \mathrm{M}$ & Basalt near Gold Digger Pass & $o b$ & $910 \pm 49$ & $\begin{array}{l}906 \pm 53 \\
921 \pm 82\end{array}$ & $0.720 \pm 0.004$ & 4 & $\begin{array}{c}18.09 \\
19.266\end{array}$ & $\begin{array}{l}9.398 \times 10^{-13} \\
9.553 \times 10^{-13}\end{array}$ & $\begin{array}{l}7.2 \\
4.4\end{array}$ & & 47.05 & 37.03 \\
\hline \multirow[t]{3}{*}{$460 \mathrm{M}$} & $\begin{array}{l}\text { Older andesite of Pumice Stone } \\
\text { Mountain }\end{array}$ & oap & $928 \pm 42$ & $928 \pm 42$ & $1.318 \pm 0.007$ & 5 & 8.3488 & $1.760 \times 10^{-12}$ & 11.5 & & 34.80 & 43.78 \\
\hline & second sample & & & $\begin{array}{l}1.02 \pm 0.03 \mathrm{Ma} \\
914 \pm 23\end{array}$ & $1.143 \pm 0.010$ & 4 & $\begin{array}{l}14.9486 \\
15.5836\end{array}$ & $\begin{array}{l}1.680 \times 10^{-12} \\
1.504 \times 10^{-12}\end{array}$ & $\begin{array}{l}19.5 \\
23.5\end{array}$ & & & \\
\hline & $\begin{array}{l}\text { weighted avg. of } 3 \\
\text { determinations }\end{array}$ & & $949 \pm 29$ & & & & & & & & & \\
\hline $469 \mathrm{M}$ & $\begin{array}{l}\text { Older rhyolite of Red Cap } \\
\text { Mountain }\end{array}$ & orr & $965 \pm 24$ & $\begin{array}{c}1.031 \pm 0.025 \mathrm{Ma} \\
905 \pm 24\end{array}$ & $3.610 \pm 0.018$ & 2 & $\begin{array}{l}6.1974 \\
9.7439\end{array}$ & $\begin{array}{l}5.358 \times 10^{-12} \\
4.703 \times 10^{-12}\end{array}$ & $\begin{array}{l}31.4 \\
58.4\end{array}$ & $\begin{array}{l}1.01 \pm 0.05 \mathrm{Ma} \text { (Mertzman, 1982, } \\
\text { no.7) }\end{array}$ & 33.48 & 44.29 \\
\hline $526 \mathrm{M}$ & $\begin{array}{l}\text { Basaltic andesite dike near } \\
\text { Winema Farms }\end{array}$ & & $3.12 \pm 0.09 \mathrm{Ma}$ & $\begin{array}{l}3.06 \pm 0.09 \mathrm{Ma} \\
3.17 \pm 0.09 \mathrm{Ma}\end{array}$ & $1.180 \pm 0.011$ & 5 & $\begin{array}{c}6.5627 \\
10.2604\end{array}$ & $\begin{array}{l}5.205 \times 10^{-12} \\
5.397 \times 10^{-12}\end{array}$ & $\begin{array}{l}43.9 \\
14.6\end{array}$ & & 55.32 & 33.95 \\
\hline $639 \mathrm{M}$ & Basalt of High Rim & & $3.60 \pm 0.12 \mathrm{Ma}$ & $\begin{array}{l}3.73 \pm 0.14 \mathrm{Ma} \\
3.54 \pm 0.10 \mathrm{Ma}\end{array}$ & $1.218 \pm 0.011$ & 5 & $\begin{array}{l}3.3517 \\
6.4251\end{array}$ & $\begin{array}{l}6.538 \pm 10^{-12} \\
6.221 \pm 10^{-12}\end{array}$ & $\begin{array}{l}12.9 \\
14.8\end{array}$ & & 55.35 & 34.54 \\
\hline $776 \mathrm{M}$ & $\begin{array}{l}\text { Older basaltic andesite of Timber } \\
\text { Mountain }\end{array}$ & omt & $1.820 \pm 0.042 \mathrm{Ma}$ & $\begin{array}{l}1.840 \pm 0.060 \mathrm{Ma} \\
1.800 \pm 0.060 \mathrm{Ma}\end{array}$ & $0.878 \pm 0.007$ & 4 & $\begin{array}{l}10.759 \\
12.294\end{array}$ & $\begin{array}{l}2.328 \times 10^{-12} \\
2.267 \times 10^{-12}\end{array}$ & $\begin{array}{l}12.1 \\
15.3\end{array}$ & & 38.40 & 16.95 \\
\hline $1223 \mathrm{M}$ & $\begin{array}{l}\text { Older basaltic andesite of Bonita } \\
\text { Butte }\end{array}$ & om & $1.211 \pm 0.067 \mathrm{Ma}$ & $\begin{array}{l}1.160 \pm 0.150 \mathrm{Ma} \\
1.330 \pm 0.100 \mathrm{Ma} \\
0.934 \pm 0.159 \mathrm{Ma} \\
1.230 \pm 0.120 \mathrm{Ma}\end{array}$ & $1.347 \pm 0.013$ & 4 & $\begin{array}{c}12.42 \\
9.958 \\
9.864 \\
10.812\end{array}$ & $\begin{array}{l}2.245 \times 10^{-12} \\
2.588 \times 10^{-12} \\
1.812 \times 10^{-12} \\
2.377 \times 10^{-12}\end{array}$ & $\begin{array}{l}3.1 \\
5.2 \\
2.3 \\
4.1\end{array}$ & & 44.77 & 39.10 \\
\hline $1229 \mathrm{M}$ & $\begin{array}{l}\text { Older basaltic andesite of Black } \\
\text { Mountain }\end{array}$ & omb & $599 \pm 16$ & $\begin{array}{l}595 \pm 21 \\
605 \pm 24\end{array}$ & $1.086 \pm 0.008$ & 4 & $\begin{array}{l}18.769 \\
19.267\end{array}$ & $\begin{array}{l}9.315 \times 10^{-13} \\
9.461 \times 10^{-13}\end{array}$ & $\begin{array}{l}12.8 \\
10.3\end{array}$ & & 32.11 & 25.98 \\
\hline $1805 \mathrm{M}$ & Basalt of Lone Pine Butte & & $1.047 \pm 0.107 \mathrm{Ma}$ & & $0.400 \pm 0.003$ & 2 & 11.223 & $6.029 \times 10^{-13}$ & 8.5 & & 40.43 & 6.32 \\
\hline $1807 M$ & Basalt of Plum Ridge & & $629 \pm 176$ & & $0.252 \pm 0.000$ & 2 & 10.341 & $2.283 \times 10^{-13}$ & 5.8 & & 34.17 & 10.44 \\
\hline $78 \mathrm{C} 4$ & Andesite near Garner Mountain & & $52 \pm 3$ & $\begin{array}{l}51 \pm 3 \\
52 \pm 3\end{array}$ & $1.672 \pm 0.028$ & 2 & $\begin{array}{l}4.5712 \\
4.8532\end{array}$ & $\begin{array}{l}1.237 \times 10^{-12} \\
1.248 \times 10^{-12}\end{array}$ & $\begin{array}{c}12.3 \\
8.8\end{array}$ & collected by R. Luedke & 35.85 & 50.82 \\
\hline
\end{tabular}


Table 1, cont.

\begin{tabular}{|c|c|c|c|c|c|c|c|c|c|c|c|c|}
\hline Sample \# & Description & Unit & Average age & Ages & $\begin{array}{l}\text { Wt. } \% \mathrm{~K}_{2} \mathrm{O} \\
\text { used }\end{array}$ & $\begin{array}{l}\mathrm{K}_{2} \mathrm{O} \text { 's } \\
\mathrm{n}=\end{array}$ & $\begin{array}{l}\text { Weight } \\
\text { (gms) }\end{array}$ & $\begin{array}{c}{ }^{40} \mathrm{Ar} \\
(\mathrm{mole} / \mathrm{g})\end{array}$ & $\begin{array}{l}\%^{40} \mathrm{Ar} \\
\mathrm{rad}\end{array}$ & Other information & $\begin{array}{l}\text { Lat. } \\
41^{\circ} \mathrm{N}\end{array}$ & $\begin{array}{l}\text { Long. } \\
121^{\circ} \mathrm{W}\end{array}$ \\
\hline $\mathrm{CBL}$ & Basalt of Chalk Bank Landing & & $1.07 \pm 0.87 \mathrm{Ma}$ & $1.07 \pm 0.87 \mathrm{Ma}$ & $0.107 \pm 0.003$ & 4 & 15.5265 & $1.655 \times 10^{-13}$ & 0.5 & collected by D. Adam & 54.61 & 38.65 \\
\hline $93 \mathrm{CSJ} 628$ & $\begin{array}{l}\text { Andesite N.W. of Double Head } \\
\text { Mountain }\end{array}$ & & $1.540 \pm 0.140 \mathrm{Ma}$ & & $2.261 \pm 0.012$ & 4 & 9.93 & $5.016 \times 10^{-12}$ & 4.4 & collected by J. Smith & 48.34 & 11.61 \\
\hline 93CSJ681 & $\begin{array}{l}\text { Basaltic andesite of Harvey Jones } \\
\text { Butte }\end{array}$ & & $2.590 \pm 0.046 \mathrm{Ma}$ & $\begin{array}{l}2.590 \pm 0.070 \mathrm{Ma} \\
2.590 \pm 0.060 \mathrm{Ma}\end{array}$ & $0.907 \pm 0.006$ & 4 & 9.777 & $\begin{array}{l}3.384 \times 10^{-12} \\
3.398 \times 10^{-12}\end{array}$ & $\begin{array}{l}16.2 \\
23.9\end{array}$ & collected by J. Smith & 45.44 & 14.65 \\
\hline
\end{tabular}


Table 2. ${ }^{40} \mathrm{Ar} /{ }^{39} \mathrm{Ar}$ ages, Medicine Lake volcano and vicinity

All samples are whole-rock and all are in ka (except as noted)

\begin{tabular}{|c|c|c|c|c|c|c|c|c|c|}
\hline Sample \# & Description & Unit & Plateau Age & Isochron Age & $\begin{array}{c}\text { Inverse Isochron } \\
\text { Age }\end{array}$ & Lab. & Ref. & $\begin{array}{l}\text { Lat. } \\
41^{\circ} \mathrm{N}\end{array}$ & $\begin{array}{l}\text { Long. } \\
121^{\circ} \mathrm{W}\end{array}$ \\
\hline $114 \mathrm{M}$ & Andesite of Alcohol Crater & aac & $114 \pm 5$ & $113 \pm 11$ & $113 \pm 10$ & USGS & & 34.81 & 32.98 \\
\hline $160 \mathrm{M}$ & Andesite east of Six Shooter Pass & aes & $307 \pm 24$ & $311 \pm 19$ & $311 \pm 19$ & USGS & & 41.36 & 43.27 \\
\hline $194 \mathrm{M}$ & Dacite tuff of Antelope Well & dta & $171 \pm 43$ & & $149 \pm 95$ & $B G C$ & 1 & 32.58 & 43.42 \\
\hline $194 M-1$ & Dacite tuff of Antelope Well; plagioclase & dta & $128 \pm 24$ & $124 \pm 69$ & $136 \pm 37$ & USGS & & 32.58 & 43.42 \\
\hline 194M-2 & Dacite tuff of Antelope Well; plagioclase & dta & $301 \pm 25$ & $337 \pm 123$ & $347 \pm 89$ & USGS & & 32.58 & 43.42 \\
\hline 194M-3 & Dacite tuff of Antelope Well; plagioclase & dta & $241 \pm 18$ & $301 \pm 151$ & $302 \pm 96$ & USGS & & 32.58 & 43.42 \\
\hline $253 \mathrm{M}$ & Rhyolite of Grasshopper Flat & rgf & $383 \pm 1$ & $383 \pm 1$ & $383 \pm 1$ & $B G C$ & & 31.24 & 42.00 \\
\hline $383 \mathrm{M}$ & Basaltic andesite north of Lookout Butte & $\mathrm{mnl}$ & $289 \pm 13$ & $327 \pm 62$ & $327 \pm 57$ & USGS & & 40.27 & 38.64 \\
\hline $455 \mathrm{M}$ & Basaltic andesite west of Red Cap Mountain & mwr & $309 \pm 17$ & $316 \pm 25$ & $315 \pm 24$ & $B G C$ & & 34.91 & 46.53 \\
\hline $471 \mathrm{M}$ & Andesite near Devils Homestead & adh & $171 \pm 4$ & $171 \pm 14$ & $171 \pm 14$ & USGS & & 46.77 & 33.97 \\
\hline $479 \mathrm{M}$ & Andesite correlated with Typhoon Mesa & atm & $282 \pm 11$ & $274 \pm 14$ & $274 \pm 14$ & $B G C$ & & 32.11 & 46.75 \\
\hline $701 \mathrm{M}$ & Basalt of Little Sand Butte & bls & $97 \pm 13$ & $10 \pm 91$ & $13 \pm 5$ & USGS & & 40.05 & 24.17 \\
\hline $912 \mathrm{M}-\mathrm{C}$ & Mafic inclusion in dacite of the south flank & ds & $159 \pm 30$ & $144 \pm 35$ & $145 \pm 28$ & USGS & & 32.50 & 32.12 \\
\hline $928 \mathrm{M}$ & Basaltic andesite E and NE of Shotgun Peak & $\mathrm{msp}$ & $168 \pm 7$ & $153 \pm 16$ & $153 \pm 15$ & USGS & & 33.47 & 31.45 \\
\hline 999M & Andesite of the north rim & anr & $100 \pm 3$ & & & $B G C$ & 2 & 36.78 & 37.05 \\
\hline $1013 \mathrm{M}$ & Andesite of the south rim & asr & $124 \pm 3$ & $126 \pm 3$ & $126 \pm 3$ & $B G C$ & 2 & 33.67 & 34.82 \\
\hline $1076 \mathrm{M}$ & Andesite east of Grasshopper Flat & aeg & $236 \pm 33$ & $223 \pm 125$ & $211 \pm 70$ & $B G C$ & & 31.93 & 37.55 \\
\hline $1079 \mathrm{M}$ & Dacite tuff of Gillem Bluff; plagioclase & pre-MLV & $2.023 \pm 0.020 \mathrm{Ma}$ & $2.008 \pm 0.022 \mathrm{Ma}$ & $2.011 \pm 0.022 \mathrm{Ma}$ & USGS & & 47.30 & 33.92 \\
\hline $1094 \mathrm{M}$ & Basaltic andesite under Giant Crater lava field & mug & $180 \pm 28$ & $151 \pm 40$ & $149 \pm 30$ & $B G C$ & & 31.74 & 39.67 \\
\hline $1326 \mathrm{M}$ & Basalt of Little Mount Hoffman & blh & $146 \pm 11$ & $149 \pm 22$ & $149 \pm 21$ & USGS & & 34.72 & 39.54 \\
\hline $1360 \mathrm{M}$ & Dacite east of the Callahan Flow & dec & $200 \pm 2$ & $199 \pm 4$ & $200 \pm 4$ & USGS & & 40.69 & 34.29 \\
\hline $1403 \mathrm{M}$ & Basaltic andesite of Eagle Nest Butte & men & $114 \pm 10$ & $114 \pm 34$ & $113 \pm 29$ & USGS & & 45.28 & 33.77 \\
\hline $1521 M$ & Basalt of Hovey Point & bhp & $445 \pm 25$ & $438 \pm 141$ & $439 \pm 110$ & USGS & & 49.15 & 31.35 \\
\hline $1529 M$ & Basaltic andesite north of Medicine Lake & $\mathrm{mnm}$ & $75 \pm 8$ & $103 \pm 48$ & $104 \pm 37$ & USGS & & 36.67 & 36.41 \\
\hline $1620 M$ & Andesite of Indian Butte & aib & $22 \pm 13$ & $43 \pm 48$ & $41 \pm 12$ & USGS & & 38.49 & 27.03 \\
\hline $1654 \mathrm{M}$ & Mafic inclusion in dacite S.W. of Kelley Pass & dsk & $244 \pm 20$ & $209 \pm 64$ & $220 \pm 49$ & USGS & & 39.20 & 40.83 \\
\hline $1707 \mathrm{M}$ & Rhyolite east of Glass Mountain & reg & $475 \pm 29$ & $418 \pm 55$ & $413 \pm 47$ & USGS & & 36.62 & 28.08 \\
\hline $1724 \mathrm{M}$ & Lake Basalt & bl & $126 \pm 14$ & $140 \pm 49$ & $143 \pm 39$ & USGS & & 33.97 & 29.91 \\
\hline $1797 \mathrm{M}$ & Basalt under Giant Crater lava field & bug & $445 \pm 27$ & $444 \pm 76$ & $446 \pm 68$ & USGS & & 22.17 & 35.41 \\
\hline
\end{tabular}


Table 2, cont. ${ }^{40} \mathrm{Ar} /{ }^{39} \mathrm{Ar}$ ages, Medicine Lake volcano and vicinity

\begin{tabular}{|c|c|c|c|c|c|c|c|c|c|}
\hline Sample \# & Description & Unit & Plateau Age & Isochron Age I & $\begin{array}{c}\text { Inverse Isochron } \\
\text { Age }\end{array}$ & Lab. & Ref. & $\begin{array}{l}\text { Lat. } \\
41^{\circ} \mathrm{N}\end{array}$ & $\begin{array}{l}\text { Long. } \\
121^{\circ} \mathrm{W}\end{array}$ \\
\hline $1799 M$ & Basalt of Damons Butte & $b d b$ & $144 \pm 15$ & $149 \pm 27$ & $155 \pm 23$ & USGS & & 30.64 & 18.03 \\
\hline 1919M & Basalt of Yellowjacket Butte & byb & $86 \pm 14$ & $76 \pm 21$ & $73 \pm 31$ & USGS & & 25.55 & 28.33 \\
\hline $2058 \mathrm{M}$ & Basaltic andesite of the railroad & $\mathrm{mrr}$ & $251 \pm 6$ & $248 \pm 14$ & $250 \pm 14$ & USGS & & 24.33 & 35.63 \\
\hline 93CSJ619 & Dacite of the Clear Lake Hills; plagioclase & pre-MLV & $4.278 \pm 0.022 \mathrm{Ma}$ & $3.659 \pm 0.219 \mathrm{Ma}$ & $4.076 \pm 0.272 \mathrm{Ma}$ & USGS & & 50.81 & 15.02 \\
\hline $93 \mathrm{CSJ} 625$ & Basalt south of Clear Lake Reservoir & pre-MLV & $1.086 \pm 0.026 \mathrm{Ma}$ & $1.127 \pm 0.034 \mathrm{Ma}$ & $1.128 \pm 0.034 \mathrm{Ma}$ & USGS & & 49.23 & 6.60 \\
\hline 94 CSJ780 & Rhyolite tuff of Box Canyon; plagioclase & pre-MLV & $1.006 \pm 0.025 \mathrm{Ma}$ & $1.124 \pm 0.143 \mathrm{Ma}$ & $1.139 \pm 0.126 \mathrm{Ma}$ & USGS & & 46.91 & 42.88 \\
\hline MLV-004-92 & Rhyolite near Cougar Butte & $\mathrm{rcb}$ & $437 \pm 7$ & & & BGC & & 39.68 & 27.36 \\
\hline MLV-008-92 & Lower rhyolite west of Callahan Flow = rgf & rgf & $391 \pm 2$ & & & BGC & & 41.62 & 39.10 \\
\hline MLV-014-92 & Lake Basalt (1724M site) & bl & $123 \pm 27$ & & & BGC & & 33.97 & 29.91 \\
\hline MLV-016-92 & Lake Basalt, east flank & bl & $65 \pm 10$ & $68 \pm 10$ & & BGC & 3 & 35.22 & 25.45 \\
\hline MLV-017-92 & Rhyolite of Mount Hoffman & rmh & $28 \pm 5$ & & & BGC & & 35.95 & 32.87 \\
\hline MLV-020-92 & Basalt of Mammoth Crater & bmc & $36 \pm 16$ & (=Total fusion age) & & BGC & & 41.58 & 32.73 \\
\hline MLV-021-92 & Andesite of Schonchin Butte & asb & $65 \pm 23$ & & $52 \pm 10$ & BGC & & 44.46 & 31.45 \\
\hline $84-27-94$ & Basalt of Tionesta & bt & $896 \pm 56$ & $68 \pm 163$ & $54 \pm 16$ & $B G C$ & 3 & 38.86 & 19.54 \\
\hline
\end{tabular}

Lab. column: USGS = analyses by M. Lanphere at USGS, Menlo Park CA; BGC = analyses by B. Turrin at Berkeley Geochronology Center Ref. column: 1= Herrero-Bervera and others, 1994; 2 = Donnelly-Nolan and others, 1994; 3 = Turrin, 1996 
TABLE 3. Data for ${ }^{40} \mathrm{Ar} /{ }^{39} \mathrm{Ar}$ experiments by M. Lanphere at USGS Menlo Park CA

\begin{tabular}{|c|c|c|c|c|c|c|c|c|c|c|c|}
\hline Temp $\left({ }^{\circ} \mathrm{C}\right)$ & ${ }^{40} \mathrm{Ar} /{ }^{39} \mathrm{Ar}$ & ${ }^{37} \mathrm{Ar}{ }^{39} \mathrm{Ar}$ & ${ }^{36} \mathrm{Ar} /{ }^{39} \mathrm{Ar}$ & Moles ${ }^{40} \mathrm{Ar}_{\text {rad }}$ & $\begin{array}{r}{ }^{40} \mathbf{A r}_{\text {rad }} \\
(\%)\end{array}$ & $\begin{array}{r}{ }^{39} \mathrm{Ar}_{\mathrm{Ca}} \\
(\%)\end{array}$ & $\begin{array}{r}{ }^{36} \mathrm{Ar}_{\mathrm{Ca}} \\
(\%)\end{array}$ & $\mathrm{K} / \mathrm{Ca}$ & $\begin{array}{r}{ }^{39} \mathrm{Ar} \\
(\%)\end{array}$ & $\begin{array}{l}\text { Age } \\
\text { (Ma) }\end{array}$ & Std.dev. \\
\hline
\end{tabular}

114M Andesite of Alcohol Crater[J=0.00033896]

plateau age $=114 \pm 5 \mathrm{ka}$; isochron age $=113 \pm 11 \mathrm{ka}$; inverse age $=113 \pm 10 \mathrm{ka}$; isochron intercept=295.6 \pm 1.2 ; total gas age $=133 \pm 5 \mathrm{ka}$

\begin{tabular}{|c|c|c|c|c|c|c|c|c|c|c|c|}
\hline 550 & 16.031 & 6.399 & 0.05861 & $-1.78 \mathrm{E}-16$ & -4.7 & 0.45 & 3.1 & 0.076 & 0.2 & $-0.466 \pm$ & 0.521 \\
\hline 600 & 3.649 & 1.68 & 0.012263 & $1.69 \mathrm{E}-15$ & 4.5 & 0.12 & 3.8 & 0.291 & 8 & $0.101 \pm$ & 0.015 \\
\hline 650 & 2.601 & 1.8217 & 0.008647 & 4.39E-15 & 7.6 & 0.13 & 5.9 & 0.269 & 17.3 & $0.121 \pm$ & 0.009 \\
\hline 700 & 2.722 & 1.8299 & 0.009142 & $3.83 E-15$ & 6.3 & 0.13 & 5.6 & 0.267 & 17.3 & $0.105 \pm$ & 0.009 \\
\hline 750 & 3.764 & 2.105 & 0.012639 & $3.05 E-15$ & 5.4 & 0.15 & 4.7 & 0.232 & 11.6 & $0.124 \pm$ & 0.012 \\
\hline 800 & 4.883 & 2.545 & 0.016563 & $2.88 \mathrm{E}-15$ & 4.1 & 0.18 & 4.3 & 0.192 & 11.2 & $0.123 \pm$ & 0.014 \\
\hline 850 & 6.588 & 1.9867 & 0.0224 & 1.17E-15 & 2 & 0.14 & 2.5 & 0.246 & 6.9 & $0.08 \pm$ & 0.02 \\
\hline 900 & 7.564 & 2.367 & 0.02545 & $1.81 \mathrm{E}-15$ & 3.2 & 0.17 & 2.6 & 0.207 & 5.9 & $0.147 \pm$ & 0.023 \\
\hline 950 & 10.344 & 2.102 & 0.0351 & $9.82 \mathrm{E}-16$ & 1.4 & 0.15 & 1.7 & 0.233 & 5.2 & $0.09 \pm$ & 0.029 \\
\hline 1000 & 12.849 & 2.485 & 0.04344 & $1.41 \mathrm{E}-15$ & 1.7 & 0.18 & 1.6 & 0.197 & 5 & $0.135 \pm$ & 0.034 \\
\hline 1050 & 13.158 & 6.446 & 0.04469 & $3.94 \mathrm{E}-15$ & 3.7 & 0.46 & 4.1 & 0.076 & 6.3 & $0.299 \pm$ & 0.036 \\
\hline 1100 & 7.229 & 6.747 & 0.02489 & $2.13 E-15$ & 6 & 0.48 & 7.6 & 0.072 & 3.8 & $0.266 \pm$ & 0.032 \\
\hline 1150 & 8.867 & 7.15 & 0.03026 & 8.48E-16 & 5.9 & 0.5 & 6.6 & 0.068 & 1.3 & $0.32 \pm$ & 0.078 \\
\hline
\end{tabular}

160M Andesite east of Six Shooter Pass[ $\mathrm{J}=\mathbf{0} . \mathbf{0 0 0 4 0 4 1}]$

plateau age $=307 \pm 24 \mathrm{ka}$; isochron age $=311 \pm 19 \mathrm{ka}$; inverse age $=311 \pm 19 \mathrm{ka}$; isochron intercept=295.4 \pm 0.4 ; total gas age $=522 \pm 57 \mathrm{ka}$

\begin{tabular}{|c|c|c|c|c|c|c|c|c|c|c|c|}
\hline 500 & 15.528 & 1.27 & 0.0508 & 1.33E-14 & 4 & 0.09 & 0.7 & 0.385 & 16.2 & $0.454 \pm$ & 0.046 \\
\hline 550 & 12.498 & 1.6032 & 0.04133 & $9.65 \mathrm{E}-15$ & 3.4 & 0.11 & 1.1 & 0.305 & 17.4 & $0.306 \pm$ & 0.039 \\
\hline 600 & 13.62 & 2.073 & 0.04522 & $1.30 \mathrm{E}-14$ & 3.2 & 0.15 & 1.3 & 0.236 & 22.9 & $0.314 \pm$ & 0.04 \\
\hline 650 & 17.476 & 3.05 & 0.05856 & $1.08 \mathrm{E}-14$ & 2.4 & 0.22 & 1.5 & 0.16 & 19.2 & $0.31 \pm$ & 0.052 \\
\hline 700 & 29.81 & 3.907 & 0.10078 & $6.02 \mathrm{E}-15$ & 1.2 & 0.28 & 1.1 & 0.125 & 12.8 & $0.26 \pm$ & 0.089 \\
\hline 750 & 62.55 & 5.205 & 0.2115 & $4.40 \mathrm{E}-15$ & 0.8 & 0.37 & 0.69 & 0.094 & 6.9 & $0.354 \pm$ & 0.187 \\
\hline 800 & 152.22 & 5.879 & 0.518 & $-7.98 \mathrm{E}-16$ & -0.2 & 0.42 & 0.32 & 0.083 & 1.6 & $-0.273 \pm$ & 0.462 \\
\hline 850 & 253.7 & 5.319 & 0.8617 & $-7.20 \mathrm{E}-16$ & -0.2 & 0.38 & 0.17 & 0.092 & 1.1 & $-0.371 \pm$ & 0.759 \\
\hline 900 & 499.6 & 10.273 & 1.6765 & $3.54 \mathrm{E}-15$ & 1 & 0.73 & 0.17 & 0.047 & 0.5 & $3.67 \pm$ & 2.06 \\
\hline 950 & 1196.4 & 20.16 & 4.01 & $3.72 \mathrm{E}-15$ & 1.1 & 1.4 & 0.14 & 0.024 & 0.2 & $9.64 \pm$ & 10.84 \\
\hline 1000 & 1555.4 & 49 & 5.193 & $8.43 \mathrm{E}-15$ & 1.6 & 3.5 & 0.27 & 0.0097 & 0.2 & $18.71 \pm$ & 12.15 \\
\hline 1050 & 724.4 & 48.53 & 2.453 & $2.42 \mathrm{E}-15$ & 0.5 & 3.4 & 0.56 & 0.0098 & 0.5 & $2.72 \pm$ & 3.21 \\
\hline
\end{tabular}


TABLE 3. Cont.

\begin{tabular}{|c|c|c|c|c|c|c|c|c|c|c|c|}
\hline Temp $\left({ }^{\circ} \mathrm{C}\right)$ & ${ }^{40} \mathrm{Ar} /{ }^{39} \mathrm{Ar}$ & ${ }^{37} \mathrm{Ar} r^{39} \mathrm{Ar}$ & ${ }^{36} \mathrm{Ar} r^{39} \mathrm{Ar}$ & Moles ${ }^{40} \mathrm{Ar}_{\mathrm{rad}}$ & $\begin{array}{r}{ }^{40} \mathrm{Ar}_{\mathrm{rad}} \\
(\%) \\
\end{array}$ & $\begin{array}{r}{ }^{39} \mathrm{Ar}_{\mathrm{Ca}} \\
(\%) \\
\end{array}$ & $\begin{array}{c}{ }^{36} \mathrm{Ar}_{\mathrm{Ca}} \\
(\%)\end{array}$ & $\mathrm{K} / \mathrm{Ca}$ & $\begin{array}{r}{ }^{39} \mathrm{Ar} \\
(\%) \\
\end{array}$ & $\begin{array}{l}\text { Age } \\
\text { (Ma) }\end{array}$ & Std.dev \\
\hline 1100 & 902.6 & 40.84 & 2.947 & $9.12 \mathrm{E}-15$ & 3.9 & 2.9 & 0.39 & 0.0117 & 0.2 & $26.13 \pm$ & 8.96 \\
\hline 1150 & 465.7 & 34.54 & 1.4728 & $5.93 \mathrm{E}-15$ & 7.2 & 2.4 & 0.66 & 0.0138 & 0.1 & $24.73 \pm$ & 7.04 \\
\hline 1250 & 973 & 28.74 & 3.008 & 4.03E-15 & 8.9 & 2 & 0.27 & 0.0167 & 0 & $63.29 \pm$ & 50.36 \\
\hline 1400 & 189.84 & 23.45 & 0.474 & $1.56 \mathrm{E}-15$ & 27.2 & 1.7 & 1.4 & 0.021 & 0 & $37.92 \pm$ & 21.65 \\
\hline
\end{tabular}

\section{M-1 Plagioclase in dacite tuff of Antelope Well [ $\mathrm{J}=\mathbf{0 . 0 0 0 3 5 6 5 1 ]}$}

plateau age $=128 \pm 24 \mathrm{ka}$; isochron age $=124 \pm 63 \mathrm{ka}$; inverse age=136 $\pm 37 \mathrm{ka}$; isochron intercept=295.0 \pm 7.6 ; total gas age $=834 \pm 28 \mathrm{ka}$

\begin{tabular}{|c|c|c|c|c|c|c|c|c|c|c|c|}
\hline 450 & 140.19 & 3.144 & 0.4175 & $1.46 \mathrm{E}-16$ & 12.2 & 0.22 & 0.21 & 0.155 & 0 & $10.99 \pm$ & 15.56 \\
\hline 500 & 280.7 & 0.06418 & 0.006393 & $6.19 \mathrm{E}-15$ & 99.3 & 0.005 & 0.28 & 7.63 & 0.1 & $170.94 \pm$ & 6.22 \\
\hline 550 & 122.97 & 0.012216 & 0.001216 & $1.43 \mathrm{E}-14$ & 99.7 & 0.001 & 0.28 & 40.1 & 0.6 & $77.18 \pm$ & 0.98 \\
\hline 600 & 43.47 & 18.335 & 0.15266 & $-1.87 \mathrm{E}-17$ & -0.3 & 1.3 & 3.4 & 0.026 & 0.8 & $-0.078 \pm$ & 0.718 \\
\hline 601 & 21.86 & 20.01 & 0.07988 & $-2.32 \mathrm{E}-17$ & -0.4 & 1.4 & 7 & 0.024 & 1.4 & $-0.056 \pm$ & 0.408 \\
\hline 650 & 10.137 & 20.8 & 0.03575 & $5.25 \mathrm{E}-16$ & 12.8 & 1.5 & 16.3 & 0.023 & 2.1 & $0.848 \pm$ & 0.275 \\
\hline 700 & 6.277 & 20.84 & 0.02524 & $3.22 \mathrm{E}-16$ & 8.8 & 1.5 & 23.2 & 0.023 & 3 & $0.358 \pm$ & 0.191 \\
\hline 750 & 5.553 & 20.27 & 0.02547 & $-2.07 E-16$ & -5.2 & 1.4 & 22.4 & 0.024 & 3.6 & $-0.19 \pm$ & 0.158 \\
\hline 800 & 3.858 & 20.96 & 0.018788 & $4.41 \mathrm{E}-17$ & 1.2 & 1.5 & 31.4 & 0.023 & 4.8 & $0.03 \pm$ & 0.122 \\
\hline 850 & 3.674 & 20.81 & 0.018034 & $8.84 \mathrm{E}-17$ & 2 & 1.5 & 32.4 & 0.023 & 6.2 & $0.047 \pm$ & 0.098 \\
\hline 900 & 3.106 & 20.45 & 0.016157 & $5.23 \mathrm{E}-17$ & 1 & 1.4 & 35.6 & 0.024 & 9.1 & $0.019 \pm$ & 0.072 \\
\hline 950 & 2.776 & 20.51 & 0.014277 & $5.39 \mathrm{E}-16$ & 9.4 & 1.4 & 40.4 & 0.024 & 10.6 & $0.169 \pm$ & 0.064 \\
\hline 1000 & 3.17 & 19.981 & 0.015301 & $7.81 \mathrm{E}-16$ & 9.7 & 1.4 & 36.7 & 0.024 & 12.9 & $0.201 \pm$ & 0.056 \\
\hline 1050 & 3.503 & 19.646 & 0.016437 & $6.74 \mathrm{E}-16$ & 7.9 & 1.4 & 33.6 & 0.025 & 12.4 & $0.181 \pm$ & 0.057 \\
\hline 1100 & 3.646 & 19.407 & 0.017187 & $4.02 \mathrm{E}-16$ & 4.9 & 1.4 & 31.7 & 0.025 & 11.5 & $0.117 \pm$ & 0.06 \\
\hline 1150 & 3.158 & 18.309 & 0.015715 & 4.70E-17 & 1.1 & 1.3 & 32.7 & 0.026 & 7 & $0.022 \pm$ & 0.087 \\
\hline 1200 & 4.416 & 17.383 & 0.018524 & $2.58 \mathrm{E}-16$ & 8.7 & 1.2 & 26.4 & 0.028 & 3.4 & $0.251 \pm$ & 0.166 \\
\hline 1250 & 10.741 & 13.483 & 0.03953 & $1.72 \mathrm{E}-16$ & 1.7 & 0.95 & 9.6 & 0.036 & 4.9 & $0.116 \pm$ & 0.119 \\
\hline 1300 & 8.685 & 12.23 & 0.03058 & 7.07E-16 & 7.7 & 0.86 & 11.2 & 0.04 & 5.5 & $0.432 \pm$ & 0.107 \\
\hline
\end{tabular}


TABLE 3. Cont.

\begin{tabular}{|c|c|c|c|c|c|c|c|c|c|c|c|}
\hline Temp $\left({ }^{\circ} \mathrm{C}\right)$ & ${ }^{40} \mathrm{Ar} /{ }^{39} \mathrm{Ar}$ & ${ }^{37} \mathrm{Ar} /{ }^{39} \mathrm{Ar}$ & ${ }^{36} \mathrm{Ar} /{ }^{39} \mathrm{Ar}$ & Moles ${ }^{40} \mathrm{Ar}_{\text {rad }}$ & $\begin{array}{r}{ }^{40} \mathrm{Ar}_{\mathrm{rad}} \\
(\%) \\
\end{array}$ & $\begin{array}{r}{ }^{39} \mathrm{Ar}_{\mathrm{Ca}} \\
(\%) \\
\end{array}$ & $\begin{array}{c}{ }^{36} \mathrm{Ar}_{\mathrm{ca}} \\
(\%)\end{array}$ & $\mathrm{K} / \mathrm{Ca}$ & $\begin{array}{r}{ }^{39} \mathbf{A r} \\
(\%) \\
\end{array}$ & $\begin{array}{l}\text { Age } \\
(\mathrm{Ma})\end{array}$ & Std.dev \\
\hline
\end{tabular}

\section{M-2 Plagioclase in dacite tuff of Antelope Well [ $\mathrm{J}=\mathbf{0 . 0 0 0 3 4 7 1 0 ]}$}

plateau age $=301 \pm 25 \mathrm{ka}$; isochron age $=337 \pm 123 \mathrm{ka}$; inverse age $=347 \pm 89 \mathrm{ka}$; isochron intercept=291.9 \pm 7.6 ; total gas age $=301 \pm 39 \mathrm{ka}$

\begin{tabular}{|c|c|c|c|c|c|c|c|c|c|c|c|}
\hline 500 & 80.18 & 0.02686 & 0.724 & $-1.70 \mathrm{E}-16$ & -166.8 & 0.002 & 0.001 & 18.2 & 0 & $-85.77 \pm$ & 111.8 \\
\hline 550 & 341.9 & 18.344 & 1.2113 & $-9.73 \mathrm{E}-17$ & -4.2 & 1.3 & 0.43 & 0.026 & 0 & $-9.22 \pm$ & 32.08 \\
\hline 600 & 549.4 & 22.48 & 1.8321 & $3.42 \mathrm{E}-16$ & 1.8 & 1.6 & 0.34 & 0.021 & 0.2 & $6.29 \pm$ & 8.94 \\
\hline 650 & 111.56 & 22.72 & 0.3919 & $-2.74 \mathrm{E}-16$ & -2.1 & 1.6 & 1.6 & 0.021 & 0.7 & $-1.5 \pm$ & 1.075 \\
\hline 700 & 34.76 & 22.84 & 0.12148 & $2.38 \mathrm{E}-16$ & 2.2 & 1.6 & 5.3 & 0.021 & 1.9 & $0.483 \pm$ & 0.354 \\
\hline 750 & 18.894 & 22.51 & 0.06814 & $2.96 \mathrm{E}-16$ & 3.3 & 1.6 & 9.3 & 0.021 & 2.9 & $0.399 \pm$ & 0.235 \\
\hline 800 & 11.816 & 21.41 & 0.04374 & $6.03 \mathrm{E}-16$ & 5.7 & 1.5 & 13.8 & 0.023 & 5.5 & $0.425 \pm$ & 0.128 \\
\hline 850 & 8.708 & 20.88 & 0.03367 & $5.70 \mathrm{E}-16$ & 5.6 & 1.5 & 17.4 & 0.023 & 7.1 & $0.312 \pm$ & 0.101 \\
\hline 900 & 6.425 & 20.82 & 0.02407 & $1.60 \mathrm{E}-15$ & 16.2 & 1.5 & 24.3 & 0.023 & 9.3 & $0.662 \pm$ & 0.08 \\
\hline 950 & 4.606 & 20.67 & 0.019742 & $1.05 \mathrm{E}-15$ & 10.6 & 1.5 & 29.4 & 0.023 & 13.1 & $0.31 \pm$ & 0.062 \\
\hline 1000 & 7.279 & 20.4 & 0.0291 & $1.00 \mathrm{E}-15$ & 5.1 & 1.4 & 19.7 & 0.024 & 16.3 & $0.238 \pm$ & 0.056 \\
\hline 1050 & 9.369 & 20.11 & 0.03668 & $5.89 \mathrm{E}-16$ & 2.1 & 1.4 & 15.4 & 0.024 & 18 & $0.126 \pm$ & 0.055 \\
\hline 1100 & 4.694 & 19.348 & 0.019577 & 1.27E-15 & 11 & 1.4 & 27.8 & 0.025 & 15 & $0.327 \pm$ & 0.056 \\
\hline 1150 & 5.33 & 18.598 & 0.02377 & $-1.60 \mathrm{E}-16$ & -2.8 & 1.3 & 22 & 0.026 & 6.5 & $-0.096 \pm$ & 0.107 \\
\hline 1200 & 8.72 & 18.782 & 0.03555 & $-1.31 \mathrm{E}-16$ & -2.6 & 1.3 & 14.8 & 0.026 & 3.5 & $-0.143 \pm$ & 0.19 \\
\hline
\end{tabular}

194M-3 Plagioclase in dacite tuff of Antelope Well [ $\mathrm{J}=0.00032718]$

plateau age $=241 \pm 18 \mathrm{ka}$; isocgron age $=301 \pm 151 \mathrm{ka}$; inverse age $=302 \pm 96 \mathrm{ka}$; isochron intercept=290.5 \pm 12.2 ; total gas age $=685 \pm 33 \mathrm{ka}$

\begin{tabular}{|c|c|c|c|c|c|c|c|c|c|c|c|}
\hline 600 & 1455 & 12.455 & 4.887 & $4.57 \mathrm{E}-16$ & 0.8 & 0.88 & 0.072 & 0.039 & 0.1 & $7.13 \pm$ & 0.45 \\
\hline 700 & 141.77 & 22.99 & 0.6039 & $-5.11 \mathrm{E}-14$ & -24.5 & 1.6 & 1.1 & 0.021 & 4.5 & $-20.98 \pm$ & 0.4 \\
\hline 725 & 10.326 & 24.74 & 0.04207 & $-4.91 \mathrm{E}-17$ & -0.5 & 1.7 & 16.5 & 0.0195 & 3 & $-0.03 \pm$ & 0.114 \\
\hline 775 & 6.781 & 23.51 & 0.02931 & $9.73 \mathrm{E}-17$ & 1.1 & 1.7 & 22.5 & 0.02 & 4 & $0.044 \pm$ & 0.086 \\
\hline 825 & 5.729 & 22.62 & 0.02411 & $6.30 \mathrm{E}-16$ & 8.4 & 1.6 & 26.4 & 0.021 & 4 & $0.29 \pm$ & 0.087 \\
\hline 875 & 5.555 & 22.2 & 0.02351 & $1.26 \mathrm{E}-15$ & 8.1 & 1.6 & 26.5 & 0.022 & 8.5 & $0.27 \pm$ & 0.052 \\
\hline 925 & 5.83 & 21.8 & 0.02464 & $1.20 \mathrm{E}-15$ & 6.1 & 1.5 & 24.9 & 0.022 & 10.2 & $0.214 \pm$ & 0.048 \\
\hline 975 & 6.837 & 21.68 & 0.02763 & 2.03E-15 & 6.9 & 1.5 & 22.1 & 0.022 & 13.1 & $0.283 \pm$ & 0.044 \\
\hline 1025 & 6.714 & 21.53 & 0.02739 & $2.02 \mathrm{E}-15$ & 6.1 & 1.5 & 22.1 & 0.022 & 15 & $0.244 \pm$ & 0.042 \\
\hline 1075 & 6.898 & 20.67 & 0.02796 & $1.86 \mathrm{E}-15$ & 5.1 & 1.5 & 20.8 & 0.023 & 16.1 & $0.211 \pm$ & 0.041 \\
\hline 1125 & 6.628 & 19.438 & 0.02665 & $1.28 \mathrm{E}-15$ & 5.5 & 1.4 & 20.5 & 0.025 & 10.6 & $0.219 \pm$ & 0.045 \\
\hline 1175 & 8.548 & 16.782 & 0.03159 & $1.66 \mathrm{E}-15$ & 7.1 & 1.2 & 14.9 & 0.029 & 8.3 & $0.363 \pm$ & 0.049 \\
\hline 1225 & 14.827 & 16.335 & 0.0509 & $1.05 \mathrm{E}-15$ & 7.7 & 1.2 & 9 & 0.03 & 2.8 & $0.681 \pm$ & 0.118 \\
\hline
\end{tabular}


TABLE 3. Cont.

\begin{tabular}{|c|c|c|c|c|c|c|c|c|c|c|c|}
\hline Temp $\left({ }^{\circ} \mathrm{C}\right)$ & ${ }^{40} \mathrm{Ar} /{ }^{39} \mathrm{Ar}$ & ${ }^{37} \mathrm{Ar} /{ }^{39} \mathrm{Ar}$ & ${ }^{36} \mathrm{Ar}{ }^{39} \mathrm{Ar}$ & Moles ${ }^{40} \mathrm{Ar}_{\text {rad }}$ & $\begin{array}{r}{ }^{40} \mathbf{A r}_{\mathrm{rad}} \\
(\%) \\
\end{array}$ & $\begin{array}{r}{ }^{39} \mathrm{Ar}_{\mathrm{Ca}} \\
(\%)\end{array}$ & $\begin{array}{r}{ }^{36} \mathrm{Ar}_{\mathrm{Ca}} \\
(\%) \\
\end{array}$ & $\mathrm{K} / \mathrm{Ca}$ & $\begin{array}{l}{ }^{39} \mathbf{A r} \\
(\%)\end{array}$ & $\begin{array}{l}\text { Age } \\
(\mathrm{Ma})\end{array}$ & Std.dev. \\
\hline
\end{tabular}

\section{M Basaltic andesite north of Lookout Butte [ $\mathrm{J}=\mathbf{0 . 0 0 0 3 9 1 7 4 ]}$}

plateau age $=289 \pm 13 \mathrm{ka}$; isochron age $=327 \pm 62 \mathrm{ka}$; inverse age $=327 \pm 57 \mathrm{ka}$; isochron intercept=293.6 \pm 3.0 ; total gas age $=264 \pm 14 \mathrm{ka}$

\begin{tabular}{|c|c|c|c|c|c|c|c|c|c|c|c|}
\hline 450 & 30.53 & 0.7022 & 0.08384 & $2.21 \mathrm{E}-15$ & 19.1 & 0.05 & 0.24 & 0.697 & 0.3 & $4.11 \pm$ & 1.13 \\
\hline 500 & 16.279 & 2.771 & 0.0559 & $-4.84 \mathrm{E}-17$ & -0.1 & 0.2 & 1.4 & 0.177 & 4 & $-0.008 \pm$ & 0.067 \\
\hline 550 & 13.104 & 3.684 & 0.04619 & $-3.24 \mathrm{E}-15$ & -1.8 & 0.26 & 2.2 & 0.133 & 12.3 & $-0.169 \pm$ & 0.039 \\
\hline 600 & 11.165 & 3.872 & 0.03767 & 6.61E-15 & 3.2 & 0.27 & 2.9 & 0.126 & 16.9 & $0.251 \pm$ & 0.033 \\
\hline 650 & 9.507 & 5.051 & 0.03215 & $6.19 \mathrm{E}-15$ & 4.5 & 0.36 & 4.4 & 0.097 & 13.2 & $0.302 \pm$ & 0.03 \\
\hline 700 & 7.57 & 6.833 & 0.026 & $7.43 \mathrm{E}-15$ & 6 & 0.48 & 7.4 & 0.071 & 14.8 & $0.322 \pm$ & 0.027 \\
\hline 750 & 6.821 & 8.624 & 0.02427 & $6.05 \mathrm{E}-15$ & 5.4 & 0.61 & 10 & 0.056 & 15 & $0.26 \pm$ & 0.028 \\
\hline 775 & 8.534 & 9.78 & 0.03014 & $3.32 \mathrm{E}-15$ & 5.2 & 0.69 & 9.1 & 0.05 & 6.8 & $0.313 \pm$ & 0.042 \\
\hline 800 & 11.104 & 10.645 & 0.03898 & $2.14 \mathrm{E}-15$ & 4.2 & 0.75 & 7.7 & 0.046 & 4.1 & $0.334 \pm$ & 0.062 \\
\hline 825 & 15.517 & 11.277 & 0.05462 & $8.79 \mathrm{E}-16$ & 2 & 0.8 & 5.8 & 0.043 & 2.5 & $0.223 \pm$ & 0.095 \\
\hline 850 & 17.658 & 11.628 & 0.06025 & $1.73 \mathrm{E}-15$ & 4.6 & 0.82 & 5.4 & 0.042 & 1.9 & $0.583 \pm$ & 0.121 \\
\hline 890 & 21.73 & 11.302 & 0.07523 & $8.81 \mathrm{E}-16$ & 2 & 0.8 & 4.2 & 0.043 & 1.8 & $0.308 \pm$ & 0.129 \\
\hline 940 & 28.33 & 10.253 & 0.09715 & 7.52E-16 & 1.7 & 0.72 & 3 & 0.047 & 1.4 & $0.341 \pm$ & 0.166 \\
\hline 990 & 29.81 & 9.777 & 0.10345 & $6.91 \mathrm{E}-17$ & 0.2 & 0.69 & 2.7 & 0.05 & 1.3 & $0.034 \pm$ & 0.181 \\
\hline 1040 & 33.72 & 9.482 & 0.11187 & $2.31 \mathrm{E}-15$ & 4.3 & 0.67 & 2.4 & 0.051 & 1.4 & $1.028 \pm$ & 0.17 \\
\hline 1090 & 41.33 & 12.06 & 0.13837 & $1.96 \mathrm{E}-15$ & 3.5 & 0.85 & 2.4 & 0.04 & 1.2 & $1.03 \pm$ & 0.203 \\
\hline 1140 & 66.95 & 25.53 & 0.2269 & $1.83 \mathrm{E}-15$ & 3 & 1.8 & 3.2 & 0.0188 & 0.8 & $1.443 \pm$ & 0.314 \\
\hline
\end{tabular}

\section{M Andesite near Devils Homestead [ $\mathrm{J}=0.00035292]$}

plateau age $=171 \pm 4 \mathrm{ka}$; isochron age $=171 \pm 14 \mathrm{ka}$; inverse age $=171 \pm 14 \mathrm{ka}$; isochron intercept=295.6 \pm 1.9 ; total gas age=187 $\pm 4 \mathrm{ka}$

$\begin{array}{rrrrrrrrrrrr}550 & 48.15 & 20.71 & 0.15916 & 1.86 \mathrm{E}-16 & 5.9 & 1.5 & 3.7 & 0.023 & 0 & 1.833 \pm & 1.997 \\ 600 & 7.918 & 2.492 & 0.02604 & 2.09 \mathrm{E}-15 & 5.4 & 0.18 & 2.7 & 0.196 & 2.8 & 0.274 \pm & 0.032 \\ 650 & 4.617 & 2.413 & 0.015273 & 3.14 \mathrm{E}-15 & 6.6 & 0.17 & 4.4 & 0.203 & 6 & 0.194 \pm & 0.016 \\ 700 & 3.191 & 2.243 & 0.010501 & 6.09 \mathrm{E}-15 & 8.6 & 0.16 & 6 & 0.218 & 12.8 & 0.175 \pm & 0.01 \\ 750 & 2.74 & 2.175 & 0.008923 & 6.35 \mathrm{E}-15 & 10.4 & 0.15 & 6.8 & 0.225 & 12.9 & 0.181 \pm & 0.009 \\ 800 & 2.793 & 1.9231 & 0.009135 & 4.64 \mathrm{E}-15 & 9.1 & 0.14 & 5.9 & 0.254 & 10.6 & 0.161 \pm & 0.01 \\ 850 & 3.294 & 1.7523 & 0.010735 & 5.68 \mathrm{E}-15 & 8.1 & 0.12 & 4.6 & 0.279 & 12.3 & 0.17 \pm & 0.01 \\ 900 & 3.756 & 1.7008 & 0.012377 & 4.17 \mathrm{E}-15 & 6.4 & 0.12 & 3.9 & 0.288 & 10 & 0.153 \pm & 0.011\end{array}$


TABLE 3. Cont.

\begin{tabular}{|c|c|c|c|c|c|c|c|c|c|c|c|}
\hline $\operatorname{Temp}\left({ }^{\circ} \mathrm{C}\right)$ & ${ }^{40} \mathrm{Ar} /{ }^{39} \mathrm{Ar}$ & ${ }^{37} \mathrm{Ar} /{ }^{39} \mathrm{Ar}$ & ${ }^{36} \mathrm{Ar} /{ }^{39} \mathrm{Ar}$ & Moles ${ }^{40} \mathrm{Ar}_{\mathrm{rad}}$ & $\begin{array}{r}{ }^{40} \mathrm{Ar}_{\mathrm{rad}} \\
(\%) \\
\end{array}$ & $\begin{array}{r}{ }^{39} \mathrm{Ar}_{\mathrm{Ca}} \\
(\%) \\
\end{array}$ & $\begin{array}{c}{ }^{36} \mathrm{Ar}_{\mathrm{Ca}} \\
(\%)\end{array}$ & $\mathrm{K} / \mathrm{Ca}$ & $\begin{array}{l}{ }^{39} \mathrm{Ar} \\
(\%) \\
\end{array}$ & $\begin{array}{l}\text { Age } \\
(\mathrm{Ma}) \\
\end{array}$ & Std.dev. \\
\hline 950 & 5.244 & 1.2092 & 0.017138 & $4.06 \mathrm{E}-15$ & 5.3 & 0.085 & 2 & 0.405 & 8.4 & $0.178 \pm$ & 0.015 \\
\hline 1000 & 4.411 & 1.1437 & 0.014315 & $7.25 \mathrm{E}-15$ & 6.3 & 0.081 & 2.2 & 0.428 & 15.2 & $0.176 \pm$ & 0.012 \\
\hline 1025 & 6.856 & 2.529 & 0.02308 & $2.18 \mathrm{E}-15$ & 3.6 & 0.18 & 3.1 & 0.193 & 5.1 & $0.157 \pm$ & 0.021 \\
\hline 1050 & 15.872 & 16.01 & 0.05647 & $1.72 \mathrm{E}-15$ & 3.2 & 1.1 & 8 & 0.03 & 1.9 & $0.33 \pm$ & 0.059 \\
\hline 1075 & 12.352 & 24.83 & 0.04405 & $1.43 \mathrm{E}-15$ & 11.3 & 1.8 & 15.8 & 0.0194 & 0.6 & $0.904 \pm$ & 0.135 \\
\hline 1125 & 8.435 & 17.038 & 0.03075 & $1.74 \mathrm{E}-15$ & 9 & 1.2 & 15.6 & 0.028 & 1.3 & $0.491 \pm$ & 0.066 \\
\hline
\end{tabular}

\section{M Basalt of Little Sand Butte [J=0.00038602]}

plateau age $=97 \pm 13 \mathrm{ka}$; isochron age $=10 \pm 91 \mathrm{ka}$; inverse age $=13 \pm 5 \mathrm{ka}$; isochron intercept $=301.1 \pm 5.8$; total gas age $=31 \pm 14 \mathrm{ka}$

\begin{tabular}{|c|c|c|c|c|c|c|c|c|c|c|c|}
\hline 500 & 13.879 & 5.949 & 0.05233 & $-4.04 \mathrm{E}-15$ & -7.9 & 0.42 & 3.2 & 0.082 & 4.2 & $-0.764 \pm$ & 0.075 \\
\hline 550 & 8.721 & 6.178 & 0.03188 & $-2.31 \mathrm{E}-15$ & -2.1 & 0.44 & 5.4 & 0.079 & 14 & $-0.13 \pm$ & 0.032 \\
\hline 600 & 6.274 & 6.329 & 0.02266 & $1.30 \mathrm{E}-15$ & 1.7 & 0.45 & 7.8 & 0.077 & 14.2 & $0.072 \pm$ & 0.027 \\
\hline 650 & 5.602 & 6.215 & 0.02007 & $2.10 \mathrm{E}-15$ & 3.4 & 0.44 & 8.7 & 0.078 & 12.6 & $0.132 \pm$ & 0.028 \\
\hline 700 & 5.894 & 6.249 & 0.02157 & $5.52 \mathrm{E}-16$ & 0.7 & 0.44 & 8.1 & 0.078 & 15.6 & $0.028 \pm$ & 0.026 \\
\hline 750 & 6.722 & 6.964 & 0.02387 & $2.23 E-15$ & 3.7 & 0.49 & 8.2 & 0.07 & 10.2 & $0.173 \pm$ & 0.034 \\
\hline 775 & 8.277 & 8.488 & 0.02989 & 8.07E-16 & 1.8 & 0.6 & 8 & 0.057 & 6 & $0.105 \pm$ & 0.052 \\
\hline 800 & 10.076 & 10.488 & 0.03624 & $9.50 \mathrm{E}-16$ & 2.4 & 0.74 & 8.1 & 0.046 & 4.5 & $0.167 \pm$ & 0.068 \\
\hline 825 & 12.575 & 12.081 & 0.04547 & 3.97E-16 & 1.1 & 0.85 & 7.5 & 0.04 & 3.1 & $0.1 \pm$ & 0.094 \\
\hline 850 & 14.423 & 12.643 & 0.05128 & $7.03 E-16$ & 2.2 & 0.89 & 6.9 & 0.038 & 2.5 & $0.223 \pm$ & 0.117 \\
\hline 890 & 17.054 & 12.097 & 0.06134 & $-1.56 \mathrm{E}-16$ & -0.4 & 0.85 & 5.5 & 0.04 & 2.6 & $-0.047 \pm$ & 0.113 \\
\hline 930 & 22.08 & 10.946 & 0.07778 & $8.74 \mathrm{E}-18$ & 0 & 0.77 & 4 & 0.044 & 1.9 & $0.003 \pm$ & 0.156 \\
\hline 970 & 29.23 & 10.565 & 0.10078 & $5.86 \mathrm{E}-16$ & 1.1 & 0.75 & 2.9 & 0.046 & 2 & $0.226 \pm$ & 0.152 \\
\hline 1020 & 46.53 & 16.008 & 0.1602 & 7.39E-16 & 1.1 & 1.1 & 2.8 & 0.03 & 1.6 & $0.368 \pm$ & 0.208 \\
\hline 1120 & 73.54 & 50.34 & 0.2688 & $-2.23 E-15$ & -2.3 & 3.6 & 5.3 & 0.0094 & 1.4 & $-1.234 \pm$ & 0.287 \\
\hline 1250 & 52.35 & 58.47 & 0.19237 & $6.62 \mathrm{E}-16$ & 0.7 & 4.1 & 8.5 & 0.008 & 2 & $0.261 \pm$ & 0.224 \\
\hline 1400 & 48.46 & 51.38 & 0.17454 & $1.64 \mathrm{E}-15$ & 2.4 & 3.6 & 8.3 & 0.0092 & 1.6 & $0.83 \pm$ & 0.236 \\
\hline
\end{tabular}


TABLE 3. Cont.

\begin{tabular}{|c|c|c|c|c|c|c|c|c|c|c|c|}
\hline $\operatorname{Temp}\left({ }^{\circ} \mathrm{C}\right)$ & ${ }^{40} \mathrm{Ar} /{ }^{39} \mathrm{Ar}$ & ${ }^{37} \mathrm{Ar} /{ }^{39} \mathrm{Ar}$ & ${ }^{36} \mathrm{Ar} /{ }^{39} \mathrm{Ar}$ & Moles ${ }^{40} \mathrm{Ar}_{\text {rad }}$ & $\begin{array}{r}{ }^{40} \mathrm{Ar}_{\mathrm{rad}} \\
(\%)\end{array}$ & $\begin{array}{r}{ }^{39} \mathrm{Ar}_{\mathrm{Ca}} \\
(\%)\end{array}$ & ${ }^{36} \mathrm{Ar}_{\mathrm{Ca}}$ & $\mathrm{K} / \mathrm{Ca}$ & ${ }^{39} \mathrm{~A}$ & Ag & Std.dev. \\
\hline
\end{tabular}

\section{M-c Mafic inclusion in dacite of the south flank [ $\mathrm{J}=\mathbf{0 . 0 0 0 3 7 2 0 2 ]}$}

plateau age $=159 \pm 30 \mathrm{ka}$; isochron age $=144 \pm 35 \mathrm{ka}$; inverse age $=145 \pm 28 \mathrm{ka}$; isochron intercept=295.9 \pm 0.7 ; total gas age $=159 \pm 59 \mathrm{ka}$

\begin{tabular}{|c|c|c|c|c|c|c|c|c|c|c|c|}
\hline 450 & 1229.1 & 12.789 & 4.291 & $-4.23 \mathrm{E}-16$ & -3.1 & 0.9 & 0.084 & 0.038 & 0 & $-25.88 \pm$ & 233.5 \\
\hline 500 & 31.83 & 8.713 & 0.11015 & 8.58E-18 & 0 & 0.62 & 2.2 & 0.056 & 2 & $0.005 \pm$ & 0.397 \\
\hline 550 & 19.821 & 9.639 & 0.06924 & 4.97E-16 & 0.8 & 0.68 & 3.9 & 0.05 & 5.7 & $0.109 \pm$ & 0.149 \\
\hline 600 & 10.823 & 10.92 & 0.03869 & $2.70 \mathrm{E}-15$ & 2.7 & 0.77 & 7.9 & 0.045 & 16.7 & $0.201 \pm$ & 0.058 \\
\hline 650 & 10.274 & 12.431 & 0.03725 & $2.21 \mathrm{E}-15$ & 2.9 & 0.88 & 9.4 & 0.039 & 13.7 & $0.202 \pm$ & 0.067 \\
\hline 700 & 10.75 & 13.603 & 0.03967 & $1.09 \mathrm{E}-15$ & 1.5 & 0.96 & 9.6 & 0.036 & 12.8 & $0.107 \pm$ & 0.072 \\
\hline 750 & 13.866 & 13.938 & 0.05048 & $6.02 \mathrm{E}-16$ & 0.8 & 0.98 & 7.8 & 0.035 & 10.4 & $0.072 \pm$ & 0.088 \\
\hline 825 & 28.74 & 13.925 & 0.10039 & $1.58 \mathrm{E}-15$ & 0.8 & 0.98 & 3.9 & 0.035 & 12.7 & $0.155 \pm$ & 0.097 \\
\hline 900 & 38.61 & 14.766 & 0.13429 & $7.02 \mathrm{E}-16$ & 0.4 & 1 & 3.1 & 0.033 & 8.6 & $0.102 \pm$ & 0.135 \\
\hline 975 & 32.88 & 13.459 & 0.11335 & $1.23 \mathrm{E}-15$ & 1.5 & 0.95 & 3.3 & 0.036 & 4.5 & $0.341 \pm$ & 0.195 \\
\hline 1075 & 31.86 & 14.443 & 0.11122 & $8.44 \mathrm{E}-16$ & 0.6 & 1 & 3.6 & 0.034 & 7.8 & $0.135 \pm$ & 0.131 \\
\hline 1200 & 101.54 & 77.7 & 0.3635 & $1.73 \mathrm{E}-15$ & 0.6 & 5.5 & 6 & 0.006 & 5.2 & $0.417 \pm$ & 0.337 \\
\hline
\end{tabular}

928M Basaltic andesite E. and N.E. of Shotgun Peak [J=0.00036519]

plateau age=168 $\pm 7 \mathrm{ka}$; isochron age=153 $\pm 16 \mathrm{ka}$; inverse age=153 $\pm 15 \mathrm{ka}$; isochron intercept=297.9 \pm 2.3 ; total gas age $=177 \pm 11 \mathrm{ka}$

$\begin{array}{rrrrrrrrrrrr}450 & 715.3 & 3.141 & 2.026 & 1.73 \mathrm{E}-15 & 16.3 & 0.22 & 0.044 & 0.156 & 0 & 75.53 \pm & 88.74 \\ 500 & 9.38 & 2.975 & 0.03127 & 8.42 \mathrm{E}-16 & 4.1 & 0.21 & 2.7 & 0.164 & 1.1 & 0.255 \pm & 0.194 \\ 550 & 4.498 & 3.174 & 0.015326 & 3.42 \mathrm{E}-15 & 5.2 & 0.22 & 5.8 & 0.154 & 7.4 & 0.154 \pm & 0.031 \\ 600 & 2.673 & 2.938 & 0.008902 & 1.03 \mathrm{E}-14 & 10.7 & 0.21 & 9.3 & 0.166 & 18.3 & 0.189 \pm & 0.014 \\ 650 & 2.235 & 2.625 & 0.007557 & 6.63 \mathrm{E}-15 & 9.8 & 0.19 & 9.8 & 0.186 & 15.3 & 0.145 \pm & 0.016 \\ 700 & 2.134 & 2.42 & 0.00698 & 8.22 \mathrm{E}-15 & 12.8 & 0.17 & 9.7 & 0.202 & 15.3 & 0.18 \pm & 0.015 \\ 750 & 2.451 & 2.543 & 0.008165 & 6.33 \mathrm{E}-15 & 10.2 & 0.18 & 8.8 & 0.192 & 12.9 & 0.165 \pm & 0.018 \\ 800 & 3.129 & 2.983 & 0.01067 & 4.07 \mathrm{E}-15 & 7.1 & 0.21 & 7.9 & 0.164 & 9.3 & 0.147 \pm & 0.025 \\ 850 & 4.845 & 4.001 & 0.016896 & 2.53 \mathrm{E}-15 & 3.8 & 0.28 & 6.7 & 0.122 & 7 & 0.121 \pm & 0.033 \\ 900 & 6.337 & 5.566 & 0.02202 & 2.78 \mathrm{E}-15 & 4.6 & 0.39 & 7.1 & 0.088 & 4.8 & 0.193 \pm & 0.048 \\ 950 & 8.039 & 6.616 & 0.02765 & 2.95 \mathrm{E}-15 & 5.2 & 0.47 & 6.7 & 0.074 & 3.6 & 0.275 \pm & 0.063 \\ 1000 & 8.657 & 7.915 & 0.03103 & 6.28 \mathrm{E}-16 & 1.7 & 0.56 & 7.2 & 0.062 & 2.2 & 0.095 \pm & 0.1 \\ 1100 & 16.09 & 10.282 & 0.05577 & 2.50 \mathrm{E}-15 & 2.9 & 0.73 & 5.2 & 0.047 & 2.7 & 0.308 \pm & 0.089\end{array}$


TABLE 3. Cont.

\begin{tabular}{|c|c|c|c|c|c|c|c|c|c|c|c|}
\hline Temp $\left({ }^{\circ} \mathrm{C}\right)$ & ${ }^{40} \mathrm{Ar}{ }^{39} \mathrm{Ar}$ & ${ }^{37} \mathrm{Ar}{ }^{39} \mathrm{Ar}$ & ${ }^{36} \mathrm{Ar} /{ }^{39} \mathrm{Ar}$ & Moles ${ }^{40} \mathrm{Ar}_{\text {rad }}$ & $\begin{array}{r}{ }^{40} \mathrm{Ar}_{\text {rad }} \\
(\%)\end{array}$ & $\begin{array}{r}{ }^{39} \mathrm{Ar}_{\mathrm{Ca}} \\
(\%)\end{array}$ & $\begin{array}{r}{ }^{36} \mathrm{Ar}_{\mathrm{Ca}} \\
(\%)\end{array}$ & $\mathrm{K} / \mathrm{Ca}$ & $\begin{array}{r}{ }^{39} \mathrm{Ar} \\
(\%)\end{array}$ & $\begin{array}{l}\text { Age } \\
(\mathrm{Ma})\end{array}$ & Std.dev. \\
\hline
\end{tabular}

\section{M Plagioclase in dacite tuff of Gillem Bluff $[\mathrm{J}=\mathbf{0 . 0 0 0 3 7 8 3 1}]$}

plateau age $=2.023 \pm 0.020 \mathrm{Ma}$; isochron age $=2.008 \pm 0.022 \mathrm{Ma}$; inverse age $=2.011 \pm 0.022 \mathrm{Ma}$; intercept $=296.4 \pm 0.7 ;$ total gas age $=2.012 \pm 0.026$

\begin{tabular}{|c|c|c|c|c|c|c|c|c|c|c|c|}
\hline 450 & 599.5 & 6.54 & 2.018 & $1.10 \mathrm{E}-15$ & 0.6 & 0.42 & 0.066 & 0.075 & 0.6 & $2.47 \pm$ & 2.26 \\
\hline 500 & 130.75 & 5.79 & 0.4316 & 4.30E-15 & 2.7 & 0.37 & 0.28 & 0.084 & 2.4 & $2.44 \pm$ & 0.36 \\
\hline 550 & 11.773 & 6.531 & 0.03215 & $4.01 \mathrm{E}-15$ & 22.6 & 0.42 & 4.2 & 0.075 & 3 & $1.826 \pm$ & 0.136 \\
\hline 600 & 8.671 & 7.07 & 0.02084 & $8.44 \mathrm{E}-15$ & 33.9 & 0.46 & 7 & 0.069 & 5.7 & $2.01 \pm$ & 0.08 \\
\hline 650 & 7.737 & 7.725 & 0.017696 & $1.07 \mathrm{E}-14$ & 38.4 & 0.5 & 8.9 & 0.063 & 7.1 & $2.04 \pm$ & 0.06 \\
\hline 700 & 7.354 & 8.317 & 0.016522 & $1.30 \mathrm{E}-14$ & 40.4 & 0.54 & 10.3 & 0.059 & 8.6 & $2.04 \pm$ & 0.06 \\
\hline 750 & 7.442 & 8.989 & 0.016917 & $1.62 \mathrm{E}-14$ & 40.1 & 0.58 & 10.9 & 0.054 & 10.8 & $2.05 \pm$ & 0.05 \\
\hline 800 & 7.176 & 9.423 & 0.016299 & $1.69 \mathrm{E}-14$ & 40.8 & 0.61 & 11.9 & 0.052 & 11.4 & $2.01 \pm$ & 0.05 \\
\hline 850 & 6.533 & 9.709 & 0.014136 & $1.72 \mathrm{E}-14$ & 45 & 0.63 & 14.1 & 0.05 & 11.6 & $2.02 \pm$ & 0.05 \\
\hline 900 & 7.73 & 9.52 & 0.018505 & $1.74 \mathrm{E}-14$ & 36.7 & 0.61 & 10.5 & 0.051 & 12.1 & $1.945 \pm$ & 0.049 \\
\hline 950 & 7.322 & 9.717 & 0.016953 & $1.50 \mathrm{E}-14$ & 39.6 & 0.63 & 11.8 & 0.05 & 10.2 & $1.989 \pm$ & 0.053 \\
\hline 1000 & 8.457 & 10.039 & 0.019665 & $7.96 \mathrm{E}-15$ & 38.4 & 0.65 & 10.5 & 0.048 & 4.8 & $2.23 \pm$ & 0.09 \\
\hline 1050 & 11.092 & 9.983 & 0.02884 & $6.19 \mathrm{E}-15$ & 28.6 & 0.64 & 7.1 & 0.049 & 3.8 & $2.18 \pm$ & 0.11 \\
\hline 1100 & 10.962 & 10.04 & 0.02943 & $3.60 \mathrm{E}-15$ & 26.2 & 0.65 & 7 & 0.048 & 2.5 & $1.969 \pm$ & 0.164 \\
\hline 1150 & 10.365 & 10.082 & 0.02705 & 3.35E-15 & 28.7 & 0.65 & 7.6 & 0.048 & 2.2 & $2.05 \pm$ & 0.18 \\
\hline 1200 & 8.744 & 9.999 & 0.0214 & $3.76 \mathrm{E}-15$ & 34.6 & 0.65 & 9.6 & 0.049 & 2.5 & $2.07 \pm$ & 0.1 \\
\hline 1350 & 12.667 & 10.233 & 0.04852 & $-3.46 \mathrm{E}-16$ & -8.3 & 0.66 & 4.3 & 0.048 & 0.6 & $-0.724 \pm$ & 0.334 \\
\hline 1500 & 16.877 & 0.12773 & 0.07959 & $-2.71 \mathrm{E}-16$ & -39.3 & 0.008 & 0.033 & 3.84 & 0.1 & $-4.54 \pm$ & 2.66 \\
\hline
\end{tabular}

\section{Basalt of Little Mount Hoffman [ $\mathrm{J}=0.00036745]$}

plateau age=146 $\pm 11 \mathrm{ka}$; isochron age $=149 \pm 22 \mathrm{ka}$; inverse age $=149 \pm 21 \mathrm{ka}$; isochron intercept=295.3 \pm 1.5 ; total gas age $=248 \pm 12 \mathrm{ka}$

$\begin{array}{rrrrrrrrrrrr}450 & 93.17 & 665.3 & 0.4866 & 1.23 \mathrm{E}-17 & 4.9 & 47 & 38.4 & 0.0004 & 0 & 5.75 \pm & 106 \\ 500 & 37.69 & -20.85113 & 0.10787 & 1.53 \mathrm{E}-16 & 10.8 & -1.472 & -5.432 & -0.0238 & 0.1 & 2.67 \pm & 3.38 \\ 550 & 14.368 & 14.724 & 0.04852 & 8.78 \mathrm{E}-16 & 8.7 & 1 & 8.5 & 0.033 & 1.2 & 0.84 \pm & 0.188 \\ 600 & 6.968 & 10.767 & 0.02514 & 1.33 \mathrm{E}-15 & 6.2 & 0.76 & 12 & 0.045 & 5.4 & 0.289 \pm & 0.049 \\ 650 & 5.393 & 8.054 & 0.019302 & 1.80 \mathrm{E}-15 & 6.6 & 0.57 & 11.7 & 0.06 & 8.8 & 0.238 \pm & 0.032 \\ 700 & 4.812 & 7.09 & 0.017567 & 1.28 \mathrm{E}-15 & 4.4 & 0.5 & 11.3 & 0.069 & 10.7 & 0.14 \pm & 0.027 \\ 750 & 4.716 & 6.846 & 0.017094 & 1.72 \mathrm{E}-15 & 4.9 & 0.48 & 11.3 & 0.071 & 13 & 0.155 \pm & 0.024 \\ 800 & 5.743 & 6.007 & 0.02044 & 1.35 \mathrm{E}-15 & 3.5 & 0.42 & 8.3 & 0.081 & 11.7 & 0.134 \pm & 0.026 \\ 850 & 5.818 & 5.102 & 0.02039 & 1.13 \mathrm{E}-15 & 3.7 & 0.36 & 7 & 0.096 & 9.2 & 0.144 \pm & 0.029\end{array}$


TABLE 3. Cont.

\begin{tabular}{|c|c|c|c|c|c|c|c|c|c|c|c|}
\hline $\operatorname{Temp}\left({ }^{\circ} \mathrm{C}\right)$ & ${ }^{40} \mathrm{Ar} /{ }^{39} \mathrm{Ar}$ & ${ }^{37} \mathrm{Ar} /{ }^{39} \mathrm{Ar}$ & ${ }^{36} \mathrm{Ar} /{ }^{39} \mathrm{Ar}$ & Moles ${ }^{40} \mathrm{Ar}_{\mathrm{rad}}$ & $\begin{array}{r}{ }^{40} \mathrm{Ar}_{\mathrm{rad}} \\
(\%)\end{array}$ & $\begin{array}{r}{ }^{39} \mathrm{Ar}_{\mathrm{Ca}} \\
(\%) \\
\end{array}$ & $\begin{array}{r}{ }^{36} \mathrm{Ar}_{\mathrm{Ca}} \\
(\%) \\
\end{array}$ & $\mathrm{K} / \mathrm{Ca}$ & $\begin{array}{l}{ }^{39} \mathrm{Ar} \\
(\%) \\
\end{array}$ & $\begin{array}{l}\text { Age } \\
(\mathrm{Ma})\end{array}$ & Std.dev. \\
\hline 900 & 7.405 & 6.495 & 0.02601 & $1.21 \mathrm{E}-15$ & 3.5 & 0.46 & 7 & 0.075 & 8.3 & $0.172 \pm$ & 0.034 \\
\hline 950 & 8.566 & 7.35 & 0.03027 & $9.44 \mathrm{E}-16$ & 2.7 & 0.52 & 6.8 & 0.066 & 7.2 & $0.153 \pm$ & 0.039 \\
\hline 1000 & 9.916 & 7.498 & 0.03519 & $4.46 \mathrm{E}-16$ & 1.4 & 0.53 & 6 & 0.065 & 5.5 & $0.094 \pm$ & 0.049 \\
\hline 1075 & 10.204 & 4.956 & 0.03514 & $1.33 E-15$ & 2.3 & 0.35 & 4 & 0.099 & 10.1 & $0.154 \pm$ & 0.034 \\
\hline 1150 & 15.033 & 6.089 & 0.05088 & $1.88 \mathrm{E}-15$ & 3.3 & 0.43 & 3.4 & 0.08 & 6.6 & $0.334 \pm$ & 0.05 \\
\hline 1250 & 67.81 & 54.92 & 0.2307 & $5.73 E-15$ & 6.2 & 3.9 & 6.7 & 0.0086 & 2.3 & $2.89 \pm$ & 0.22 \\
\hline
\end{tabular}

1360M Dacite east of the Callahan Flow [ $\mathrm{J}=0.00032556]$

plateau age $=200 \pm 2 \mathrm{ka}$; isochron age $=199 \pm 4 \mathrm{ka}$; inverse age $=200 \pm 4 \mathrm{ka}$; isochron intercept $=296.0 \pm 7.6$; total gas age $=203 \pm 2 \mathrm{ka}$

\begin{tabular}{|c|c|c|c|c|c|c|c|c|c|c|}
\hline 550 & 10.698 & 0.8133 & 0.03681 & $-1.71 \mathrm{E}-17$ & -1.1 & 0.057 & 0.62 & 0.602 & $0.1 \pm$ & 0.663 \\
\hline 600 & 8.139 & 0.2386 & 0.02627 & $2.66 \mathrm{E}-16$ & 4.9 & 0.017 & 0.26 & 2.05 & $0.3 \pm$ & 0.149 \\
\hline 650 & 7.066 & 0.2218 & 0.02281 & $6.08 \mathrm{E}-16$ & 4.9 & 0.016 & 0.27 & 2.21 & $0.8 \pm$ & 0.058 \\
\hline 700 & 3.851 & 0.2073 & 0.011501 & $2.57 \mathrm{E}-15$ & 12.2 & 0.015 & 0.51 & 2.36 & $2.4 \pm$ & 0.02 \\
\hline 750 & 1.5171 & 0.2097 & 0.00386 & $3.73 E-15$ & 26 & 0.015 & 1.5 & 2.34 & $4.1 \pm$ & 0.011 \\
\hline 800 & 0.8197 & 0.2303 & 0.001626 & $4.87 \mathrm{E}-15$ & 43.7 & 0.016 & 4 & 2.13 & $5.9 \pm$ & 0.007 \\
\hline 850 & 0.578 & 0.2355 & 0.000836 & $7.79 \mathrm{E}-15$ & 60.7 & 0.017 & 7.9 & 2.08 & $9.6 \pm$ & 0.005 \\
\hline 900 & 0.4868 & 0.2438 & 0.000541 & $9.26 \mathrm{E}-15$ & 71.3 & 0.017 & 12.7 & 2.01 & $11.5 \pm$ & 0.004 \\
\hline 950 & 0.5765 & 0.2417 & 0.000887 & $9.18 \mathrm{E}-15$ & 58 & 0.017 & 7.7 & 2.03 & $11.9 \pm$ & 0.004 \\
\hline 1000 & 0.4588 & 0.2474 & 0.000461 & $9.72 \mathrm{E}-15$ & 74.8 & 0.017 & 15.1 & 1.98 & $12.2 \pm$ & 0.004 \\
\hline 1050 & 0.4572 & 0.2478 & 0.000491 & $8.68 \mathrm{E}-15$ & 72.8 & 0.017 & 14.2 & 1.98 & $11.3 \pm$ & 0.004 \\
\hline 1100 & 0.6005 & 0.2721 & 0.000957 & $8.80 \mathrm{E}-15$ & 56.7 & 0.019 & 8 & 1.8 & $11.2 \pm$ & 0.004 \\
\hline 1150 & 0.7384 & 0.2903 & 0.001466 & $6.72 \mathrm{E}-15$ & 44.6 & 0.02 & 5.6 & 1.69 & $8.8 \pm$ & 0.005 \\
\hline 1200 & 0.7212 & 0.3496 & 0.001416 & 4.45E-15 & 46 & 0.025 & 6.9 & 1.4 & $5.8 \pm$ & 0.008 \\
\hline 1250 & 0.7939 & 0.3832 & 0.00159 & $3.58 \mathrm{E}-15$ & 44.8 & 0.027 & 6.8 & 1.28 & $4.3 \pm$ & 0.01 \\
\hline
\end{tabular}

\section{M Basaltic andesite of Eagle Nest Butte [ $\mathrm{J}=0.00038161]$}

plateau age $=114 \pm 10 \mathrm{ka}$; isochron age=114 $\pm 34 \mathrm{ka}$; inverse age=113 $\pm 29 \mathrm{ka}$; isochron intercept=295.5 \pm 1.1 ; total gas age $=193 \pm 11 \mathrm{ka}$

$\begin{array}{rrrrrrrrrrrr}550 & 131.27 & 181.54 & 0.4068 & 5.77 \mathrm{E}-16 & 19.9 & 12.8 & 12.5 & 0.0024 & 0 & 20.53 \pm & 0.235 \\ 600 & 9.939 & 13.555 & 0.0365 & 1.98 \mathrm{E}-16 & 2.8 & 0.96 & 10.4 & 0.036 & 1.2 & 0.195 \pm & 0.21 \\ 650 & 5.493 & 6.653 & 0.019617 & 1.01 \mathrm{E}-15 & 4.5 & 0.47 & 9.5 & 0.073 & 6.8 & 0.172 \pm & 0.203 \\ 700 & 3.982 & 5.239 & 0.014411 & 1.61 \mathrm{E}-15 & 4 & 0.37 & 10.2 & 0.093 & 16.9 & 0.11 \pm & 0.166\end{array}$


TABLE 3. Cont.

\begin{tabular}{|c|c|c|c|c|c|c|c|c|c|c|c|}
\hline $\operatorname{Temp}\left({ }^{\circ} \mathrm{C}\right)$ & ${ }^{40} \mathrm{Ar} /{ }^{39} \mathrm{Ar}$ & ${ }^{37} \mathrm{Ar} /{ }^{39} \mathrm{Ar}$ & ${ }^{36} \mathrm{Ar} /{ }^{39} \mathrm{Ar}$ & Moles ${ }^{40} \mathrm{Ar}_{\mathrm{rad}}$ & $\begin{array}{r}{ }^{40} \mathbf{A r}_{\text {rad }} \\
(\%) \\
\end{array}$ & $\begin{array}{r}{ }^{39} \mathrm{Ar}_{\mathrm{Ca}} \\
(\%)\end{array}$ & $\begin{array}{c}{ }^{36} \mathrm{Ar}_{\mathrm{Ca}} \\
(\%)\end{array}$ & $\mathrm{K} / \mathrm{Ca}$ & $\begin{array}{l}{ }^{39} \mathrm{Ar} \\
(\%) \\
\end{array}$ & $\begin{array}{l}\text { Age } \\
(\mathrm{Ma}) \\
\end{array}$ & Std.dev. \\
\hline 750 & 3.569 & 5.845 & 0.01315 & $1.83 \mathrm{E}-15$ & 4.7 & 0.41 & 12.5 & 0.083 & 18.2 & $0.117 \pm$ & 0.164 \\
\hline 800 & 4.501 & 6.779 & 0.01661 & $2.00 \mathrm{E}-15$ & 3.5 & 0.48 & 11.5 & 0.072 & 21.5 & $0.107 \pm$ & 0.221 \\
\hline 850 & 6.855 & 8.658 & 0.02515 & $1.13 \mathrm{E}-15$ & 2.1 & 0.61 & 9.7 & 0.056 & 13.2 & $0.099 \pm$ & 0.303 \\
\hline 900 & 10.72 & 10.813 & 0.03807 & $1.59 \mathrm{E}-15$ & 3.4 & 0.76 & 8 & 0.045 & 7.2 & $0.256 \pm$ & 0.137 \\
\hline 950 & 15.338 & 8.127 & 0.05287 & $9.07 \mathrm{E}-16$ & 2.5 & 0.57 & 4.3 & 0.06 & 3.9 & $0.27 \pm$ & 0.07 \\
\hline 1000 & 21.26 & 13.654 & 0.07345 & $1.33 \mathrm{E}-15$ & 3.2 & 0.96 & 5.2 & 0.036 & 3.2 & $0.475 \pm$ & 0.091 \\
\hline 1050 & 23.54 & 16.474 & 0.08047 & $2.34 \mathrm{E}-15$ & 4.8 & 1.2 & 5.8 & 0.029 & 3.4 & $0.786 \pm$ & 0.093 \\
\hline 1100 & 28.74 & 29.11 & 0.10269 & $2.12 \mathrm{E}-15$ & 2.8 & 2.1 & 8 & 0.0165 & 4.3 & $0.569 \pm$ & 0.105 \\
\hline
\end{tabular}

\section{M Basalt of Hovey Point [ $\mathrm{J}=0.00036096]$}

plateau age $=445 \pm 25 \mathrm{ka}$; isochron age $=438 \pm 141 \mathrm{ka}$; inverse age $=439 \pm 110 \mathrm{ka}$; isochron intercept $=295.4 \pm 7.3$; total gas age $=433 \pm 36 \mathrm{ka}$

\begin{tabular}{|c|c|c|c|c|c|c|c|c|c|c|c|}
\hline 450 & 550.8 & 91.4 & 1.7269 & $3.85 \mathrm{E}-16$ & 8.7 & 6.5 & 1.5 & 0.005 & 0 & $33.17 \pm$ & 46.34 \\
\hline 500 & 25.28 & 21.35 & 0.10213 & $-5.63 E-16$ & -12.4 & 1.5 & 5.9 & 0.023 & 1.1 & $-2.07 \pm$ & 0.74 \\
\hline 550 & 13.084 & 10.514 & 0.04949 & $-4.14 \mathrm{E}-16$ & -5.1 & 0.74 & 6 & 0.046 & 3.8 & $-0.438 \pm$ & 0.216 \\
\hline 600 & 5.522 & 12.49 & 0.016864 & 1.17E-15 & 28.5 & 0.88 & 20.8 & 0.039 & 4.6 & $1.035 \pm$ & 0.18 \\
\hline 650 & 7.016 & 11.407 & 0.02392 & $1.88 \mathrm{E}-15$ & 12.8 & 0.81 & 13.4 & 0.043 & 12.9 & $0.588 \pm$ & 0.068 \\
\hline 700 & 6.284 & 11.568 & 0.02226 & $2.57 \mathrm{E}-15$ & 10.6 & 0.82 & 14.6 & 0.042 & 23.7 & $0.438 \pm$ & 0.042 \\
\hline 750 & 7.608 & 12.506 & 0.02652 & 2.64E-15 & 10.7 & 0.88 & 13.3 & 0.039 & 20.1 & $0.533 \pm$ & 0.049 \\
\hline 800 & 10.239 & 14.4 & 0.03775 & $6.80 \mathrm{E}-16$ & 2.7 & 1 & 10.7 & 0.034 & 14.8 & $0.185 \pm$ & 0.065 \\
\hline 850 & 12.848 & 19.314 & 0.04741 & $6.58 \mathrm{E}-16$ & 3.5 & 1.4 & 11.4 & 0.025 & 9.1 & $0.293 \pm$ & 0.101 \\
\hline 900 & 21.04 & 21.73 & 0.07433 & $9.61 \mathrm{E}-16$ & 4.2 & 1.5 & 8.2 & 0.022 & 6.7 & $0.579 \pm$ & 0.137 \\
\hline 950 & 34.66 & 18.296 & 0.11765 & $7.40 \mathrm{E}-16$ & 4.1 & 1.3 & 4.4 & 0.026 & 3.2 & $0.934 \pm$ & 0.269 \\
\hline
\end{tabular}

\section{M Basaltic andesite north of Medicine Lake [ $\mathrm{J}=0.0003929]$}

plateau age $=75 \pm 8 \mathrm{ka}$; isochron age $=103 \pm 48 \mathrm{ka}$; inverse age=104 $\pm 37 \mathrm{ka}$; isochron intercept=292.6 \pm 4.2 ; total gas age $=15 \pm 13 \mathrm{ka}$

\begin{tabular}{|c|c|c|c|c|c|c|c|c|c|c|c|}
\hline 450 & 78.077 & 8.419 & 0.5106 & $-1.22 \mathrm{E}-16$ & 292.4 & 0.59 & 0.46 & 0.058 & 0 & $-170.57 \pm$ & 489.6 \\
\hline 500 & 45.72 & 14.734 & 0.9293 & $1.94 \mathrm{E}-16$ & -498 & 1 & 0.45 & 0.033 & 0 & $-170.91 \pm$ & 369.3 \\
\hline 501 & 42.26 & 2.111 & 0.14546 & $-1.48 \mathrm{E}-16$ & -1.3 & 0.15 & 0.41 & 0.23 & 0.3 & $-0.389 \pm$ & 0.772 \\
\hline 550 & 4.602 & 2.124 & 0.015501 & $1.88 \mathrm{E}-15$ & 4.3 & 0.15 & 3.8 & 0.23 & 8.9 & $0.14 \pm$ & 0.025 \\
\hline 600 & 3.478 & 1.9714 & 0.011954 & $2.81 \mathrm{E}-15$ & 3.1 & 0.14 & 4.6 & 0.25 & 24.1 & $0.077 \pm$ & 0.012 \\
\hline 650 & 4.169 & 2.082 & 0.014253 & $1.59 \mathrm{E}-15$ & 3.1 & 0.15 & 4.1 & 0.23 & 11.4 & $0.092 \pm$ & 0.02 \\
\hline
\end{tabular}


TABLE 3. Cont.

\begin{tabular}{|c|c|c|c|c|c|c|c|c|c|c|c|}
\hline $\operatorname{Temp}\left({ }^{\circ} \mathrm{C}\right)$ & ${ }^{40} \mathrm{Ar} /{ }^{39} \mathrm{Ar}$ & ${ }^{37} \mathrm{Ar} /{ }^{39} \mathrm{Ar}$ & ${ }^{36} \mathrm{Ar} /{ }^{39} \mathrm{Ar}$ & Moles ${ }^{40} \mathrm{Ar}_{\mathrm{rad}}$ & $\begin{array}{r}{ }^{40} \mathrm{Ar}_{\mathrm{rad}} \\
(\%) \\
\end{array}$ & $\begin{array}{r}{ }^{39} \mathrm{Ar}_{\mathrm{Ca}} \\
(\%) \\
\end{array}$ & $\begin{array}{r}{ }^{36} \mathrm{Ar}_{\mathrm{Ca}} \\
(\%) \\
\end{array}$ & $\mathrm{K} / \mathrm{Ca}$ & $\begin{array}{l}{ }^{39} \mathbf{A r} \\
(\%) \\
\end{array}$ & $\begin{array}{l}\text { Age } \\
(\mathrm{Ma}) \\
\end{array}$ & Std.dev. \\
\hline 700 & 5.198 & 2.335 & 0.018099 & $8.22 \mathrm{E}-16$ & 0.8 & 0.16 & 3.6 & 0.21 & 17.7 & $0.031 \pm$ & 0.018 \\
\hline 750 & 8.937 & 2.408 & 0.03066 & $1.09 \mathrm{E}-15$ & 0.8 & 0.17 & 2.2 & 0.2 & 13.4 & $0.054 \pm$ & 0.027 \\
\hline 800 & 14.826 & 2.947 & 0.05149 & $-1.75 E-15$ & -1 & 0.21 & 1.6 & 0.16 & 11.2 & $-0.104 \pm$ & 0.044 \\
\hline 850 & 23.19 & 2.973 & 0.0807 & $-1.45 E-15$ & -1.8 & 0.21 & 1 & 0.16 & 3.3 & $-0.29 \pm$ & 0.083 \\
\hline 900 & 36.47 & 2.213 & 0.12648 & $-2.03 E-15$ & -2 & 0.16 & 0.49 & 0.23 & 2.6 & $-0.512 \pm$ & 0.118 \\
\hline 950 & 61.02 & 1.6164 & 0.2093 & $-2.46 E-15$ & -1.1 & 0.11 & 0.22 & 0.31 & 3.3 & $-0.492 \pm$ & 0.178 \\
\hline 975 & 79.23 & 3.573 & 0.2685 & $3.73 E-16$ & 0.2 & 0.25 & 0.37 & 0.14 & 2 & $0.126 \pm$ & 0.23 \\
\hline 1000 & 80.2 & 9.087 & 0.2715 & $1.45 \mathrm{E}-15$ & 0.9 & 0.64 & 0.94 & 0.054 & 1.9 & $0.518 \pm$ & 0.234 \\
\hline
\end{tabular}

\section{M Andesite of Indian Butte [ $\mathrm{J}=\mathbf{0 . 0 0 0 3 6 8 0 5}$ ]}

plateau age $=22 \pm 13 \mathrm{ka}$; isochron age $=43 \pm 48 \mathrm{ka}$; inverse age $=41 \pm 12 \mathrm{ka}$; isochron intercept=294.6 \pm 1.6 ; total gas age $=20 \pm 13 \mathrm{ka}$

\begin{tabular}{|c|c|c|c|c|c|c|c|c|c|c|c|}
\hline 550 & 27.58 & 13.188 & 0.09868 & $-9.43 E-17$ & -1.8 & 0.93 & 3.8 & 0.037 & 0.2 & $-0.327 \pm$ & 1.258 \\
\hline 600 & 15.094 & 4.673 & 0.05172 & $5.86 \mathrm{E}-16$ & 1.3 & 0.33 & 2.5 & 0.105 & 3.5 & $0.131 \pm$ & 0.089 \\
\hline 650 & 12.275 & 4.33 & 0.04186 & $1.61 \mathrm{E}-15$ & 2.2 & 0.31 & 2.9 & 0.113 & 7.2 & $0.177 \pm$ & 0.05 \\
\hline 700 & 9.671 & 3.887 & 0.03365 & 7.30E-16 & 0.5 & 0.27 & 3.2 & 0.126 & 17.3 & $0.034 \pm$ & 0.029 \\
\hline 725 & 8.999 & 2.743 & 0.03118 & $2.47 \mathrm{E}-16$ & 0.2 & 0.19 & 2.5 & 0.178 & 21.6 & $0.009 \pm$ & 0.026 \\
\hline 750 & 10.352 & 3.303 & 0.036 & $-1.10 \mathrm{E}-16$ & -0.1 & 0.23 & 2.6 & 0.148 & 12.2 & $-0.007 \pm$ & 0.035 \\
\hline 775 & 11.56 & 4.039 & 0.04015 & $3.09 \mathrm{E}-16$ & 0.3 & 0.29 & 2.8 & 0.121 & 12.2 & $0.02 \pm$ & 0.037 \\
\hline 800 & 15.538 & 4.82 & 0.05409 & $-3.03 E-16$ & -0.3 & 0.34 & 2.5 & 0.101 & 7.8 & $-0.031 \pm$ & 0.053 \\
\hline 825 & 22.34 & 6.421 & 0.07733 & $1.08 \mathrm{E}-16$ & 0.1 & 0.45 & 2.3 & 0.076 & 4.6 & $0.019 \pm$ & 0.083 \\
\hline 875 & 31.35 & 5.005 & 0.10937 & $-2.15 E-15$ & -1.8 & 0.35 & 1.3 & 0.098 & 4.6 & $-0.37 \pm$ & 0.098 \\
\hline 925 & 44.69 & 5.956 & 0.15299 & $-6.29 E-17$ & -0.1 & 0.42 & 1.1 & 0.082 & 3 & $-0.017 \pm$ & 0.143 \\
\hline 975 & 47.33 & 8.548 & 0.16223 & $1.78 \mathrm{E}-16$ & 0.2 & 0.6 & 1.5 & 0.057 & 2.1 & $0.067 \pm$ & 0.177 \\
\hline 1025 & 36.2 & 8.194 & 0.12314 & $9.29 \mathrm{E}-16$ & 1.3 & 0.58 & 1.9 & 0.059 & 2.3 & $0.325 \pm$ & 0.153 \\
\hline 1075 & 41.17 & 15.276 & 0.142 & $5.35 \mathrm{E}-16$ & 1.2 & 1.1 & 3 & 0.032 & 1.3 & $0.318 \pm$ & 0.238 \\
\hline
\end{tabular}

1654M Mafic inclusion in dacite southwest of Kelley Pass [ $\mathrm{J}=\mathbf{0 . 0 0 0 3 8 0 5 8 ]}$

plateau age $=244 \pm 20 \mathrm{ka}$; isochron age $=209 \pm 64 \mathrm{ka}$; inverse age $=220 \pm 49 \mathrm{ka}$; isochron intercept=297.3 \pm 3.7 ; total gas age $=298 \pm 19 \mathrm{ka}$

\begin{tabular}{|c|c|c|c|c|c|c|c|c|c|c|c|}
\hline 450 & 7135.3 & 273.9 & 24.23 & $-2.43 \mathrm{E}-17$ & 0 & 19.3 & 0.32 & 0.0014 & 0 & $-2.31 \pm$ & 642.8 \\
\hline 480 & 122.74 & 164.45 & 0.5113 & $-4.33 \mathrm{E}-16$ & -12 & 11.6 & 9 & 0.0026 & 0.1 & $-11.45 \pm$ & 6.21 \\
\hline 530 & 41.96 & 12.726 & 0.12808 & $8.28 \mathrm{E}-16$ & 12.3 & 0.9 & 2.8 & 0.038 & 0.7 & $3.58 \pm$ & 0.87 \\
\hline
\end{tabular}


TABLE 3. Cont.

\begin{tabular}{|c|c|c|c|c|c|c|c|c|c|c|c|}
\hline Temp $\left({ }^{\circ} \mathrm{C}\right)$ & ${ }^{40} \mathrm{Ar} /{ }^{39} \mathrm{Ar}$ & ${ }^{37} \mathrm{Ar} /{ }^{39} \mathrm{Ar}$ & ${ }^{36} \mathrm{Ar} /{ }^{39} \mathrm{Ar}$ & Moles ${ }^{40} \mathrm{Ar}_{\mathrm{rad}}$ & $\begin{array}{r}{ }^{40} \mathrm{Ar}_{\mathrm{rad}} \\
(\%) \\
\end{array}$ & $\begin{array}{r}{ }^{39} \mathrm{Ar}_{\mathrm{Ca}} \\
(\%) \\
\end{array}$ & $\begin{array}{r}{ }^{36} \mathrm{Ar}_{\mathrm{Ca}} \\
(\%) \\
\end{array}$ & $\mathrm{K} / \mathrm{Ca}$ & $\begin{array}{l}{ }^{39} \mathbf{A r} \\
(\%) \\
\end{array}$ & $\begin{array}{l}\text { Age } \\
(\mathrm{Ma})\end{array}$ & Std.dev. \\
\hline 580 & 10.99 & 10.727 & 0.03739 & $9.25 \mathrm{E}-16$ & 7.6 & 0.76 & 8.1 & 0.045 & 4.5 & $0.574 \pm$ & $\overline{0.126}$ \\
\hline 630 & 7.125 & 10.099 & 0.02551 & $9.30 \mathrm{E}-16$ & 6 & 0.71 & 11.1 & 0.048 & 8.9 & $0.294 \pm$ & 0.067 \\
\hline 680 & 5.656 & 9.543 & 0.02009 & $1.77 \mathrm{E}-15$ & 9.1 & 0.67 & 13.3 & 0.051 & 14.1 & $0.354 \pm$ & 0.045 \\
\hline 730 & 4.926 & 10.267 & 0.019129 & $4.59 \mathrm{E}-16$ & 2.6 & 0.72 & 15.1 & 0.047 & 14.8 & $0.087 \pm$ & 0.044 \\
\hline 780 & 4.862 & 11.927 & 0.018666 & $1.38 \mathrm{E}-15$ & 6.9 & 0.84 & 18 & 0.041 & 16.7 & $0.233 \pm$ & 0.041 \\
\hline 830 & 6.154 & 13.717 & 0.02334 & $1.22 \mathrm{E}-15$ & 6.4 & 0.97 & 16.5 & 0.035 & 12.6 & $0.273 \pm$ & 0.053 \\
\hline 880 & 10.658 & 15.507 & 0.03887 & $9.82 \mathrm{E}-16$ & 4.3 & 1.1 & 11.2 & 0.031 & 8.7 & $0.32 \pm$ & 0.075 \\
\hline 930 & 16.187 & 17.311 & 0.05848 & $4.43 E-16$ & 2.1 & 1.2 & 8.3 & 0.028 & 5.2 & $0.239 \pm$ & 0.118 \\
\hline 980 & 14.771 & 15.793 & 0.05311 & $3.15 \mathrm{E}-16$ & 2.6 & 1.1 & 8.4 & 0.031 & 3.3 & $0.269 \pm$ & 0.174 \\
\hline 1050 & 25.87 & 12 & 0.08942 & 3.85E-16 & 1.7 & 0.85 & 3.8 & 0.04 & 3.6 & $0.305 \pm$ & 0.169 \\
\hline 1125 & 25.43 & 15.658 & 0.08998 & $9.86 \mathrm{E}-17$ & 0.6 & 1.1 & 4.9 & 0.031 & 2.8 & $0.099 \pm$ & 0.208 \\
\hline 1250 & 52.61 & 119.58 & 0.2075 & $1.29 \mathrm{E}-15$ & 2.3 & 8.4 & 16.2 & 0.0038 & 3.9 & $0.922 \pm$ & 0.311 \\
\hline
\end{tabular}

1707M Rhyolite east of Glass Mountain [ $\mathrm{J}=\mathbf{0 . 0 0 0 3 6 8 8 8 ]}$

plateau age $=475 \pm 29 \mathrm{ka}$; isochron age $=418 \pm 55 \mathrm{ka}$; inverse age $=413 \pm 47 \mathrm{ka}$; isochron intercept $=319.2 \pm 23$; total gas age $=480 \pm 3 \mathrm{ka}$

\begin{tabular}{|c|c|c|c|c|c|c|c|c|c|c|c|}
\hline 450 & 3.015 & 0.06159 & 0.007329 & 4.04E-15 & 28.2 & 0.004 & 0.23 & 7.96 & 1.5 & $0.565 \pm$ & 0.027 \\
\hline 500 & 2.14 & 0.04075 & 0.004808 & $9.12 \mathrm{E}-15$ & 33.5 & 0.003 & 0.23 & 12 & 3.9 & $0.477 \pm$ & 0.009 \\
\hline 550 & 1.8797 & 0.04024 & 0.003942 & $1.84 \mathrm{E}-14$ & 37.9 & 0.003 & 0.27 & 12.2 & 8 & $0.474 \pm$ & 0.006 \\
\hline 600 & 1.7636 & 0.04374 & 0.00369 & $1.71 \mathrm{E}-14$ & 38.1 & 0.003 & 0.32 & 11.2 & 7.9 & $0.447 \pm$ & 0.006 \\
\hline 650 & 1.736 & 0.03755 & 0.00343 & 2.37E-14 & 41.5 & 0.003 & 0.29 & 13 & 10.2 & $0.479 \pm$ & 0.005 \\
\hline 700 & 1.7243 & 0.04213 & 0.003456 & $2.71 \mathrm{E}-14$ & 40.7 & 0.003 & 0.33 & 11.6 & 11.9 & $0.466 \pm$ & 0.004 \\
\hline 725 & 1.7076 & 0.04431 & 0.003387 & $2.05 \mathrm{E}-14$ & 41.3 & 0.003 & 0.35 & 11.1 & 9 & $0.469 \pm$ & 0.005 \\
\hline 750 & 1.7095 & 0.04142 & 0.003358 & $1.81 \mathrm{E}-14$ & 41.8 & 0.003 & 0.33 & 11.8 & 7.8 & $0.476 \pm$ & 0.005 \\
\hline 775 & 1.7196 & 0.03936 & 0.00345 & $1.69 \mathrm{E}-14$ & 40.6 & 0.003 & 0.31 & 12.4 & 7.5 & $0.465 \pm$ & 0.006 \\
\hline 800 & 1.7331 & 0.043 & 0.003399 & $1.55 \mathrm{E}-14$ & 42 & 0.003 & 0.34 & 11.4 & 6.6 & $0.484 \pm$ & 0.006 \\
\hline 850 & 1.7437 & 0.03833 & 0.003447 & $1.49 \mathrm{E}-14$ & 41.5 & 0.003 & 0.3 & 12.8 & 6.4 & $0.481 \pm$ & 0.006 \\
\hline 900 & 1.8666 & 0.04126 & 0.003776 & $1.52 \mathrm{E}-14$ & 40.1 & 0.003 & 0.29 & 11.9 & 6.3 & $0.498 \pm$ & 0.006 \\
\hline 950 & 1.8961 & 0.03923 & 0.003811 & $1.28 \mathrm{E}-14$ & 40.5 & 0.003 & 0.28 & 12.5 & 5.1 & $0.511 \pm$ & 0.007 \\
\hline 1000 & 1.8288 & 0.03742 & 0.003693 & 8.57E-15 & 40.2 & 0.003 & 0.27 & 13.1 & 3.6 & $0.489 \pm$ & 0.01 \\
\hline 1100 & 1.8324 & 0.02936 & 0.003478 & $7.00 \mathrm{E}-15$ & 43.8 & 0.002 & 0.23 & 16.7 & 2.7 & $0.533 \pm$ & 0.013 \\
\hline 1200 & 1.9545 & 0.009771 & 0.003919 & $3.70 \mathrm{E}-15$ & 40.5 & 0.001 & 0.067 & 50.1 & 1.4 & $0.527 \pm$ & 0.023 \\
\hline 1400 & 2.315 & 0.012291 & 0.004616 & $1.06 \mathrm{E}-15$ & 40.9 & 0.001 & 0.072 & 39.9 & 0.3 & $0.63 \pm$ & 0.094 \\
\hline
\end{tabular}


TABLE 3. Cont.

\begin{tabular}{|c|c|c|c|c|c|c|c|c|c|c|c|}
\hline Temp $\left({ }^{\circ} \mathrm{C}\right)$ & ${ }^{40} \mathrm{Ar} /{ }^{39} \mathrm{Ar}$ & ${ }^{37} \mathrm{Ar} /{ }^{39} \mathrm{Ar}$ & ${ }^{36} \mathrm{Ar} /{ }^{39} \mathrm{Ar}$ & Moles ${ }^{40} \mathrm{Ar}_{\mathrm{rad}}$ & $\begin{array}{r}{ }^{40} \mathrm{Ar}_{\mathrm{rad}} \\
(\%)\end{array}$ & $\begin{array}{r}{ }^{39} \mathrm{Ar}_{\mathrm{Ca}} \\
(\%) \\
\end{array}$ & $\begin{array}{r}{ }^{36} \mathrm{Ar}_{\mathrm{Ca}} \\
(\%) \\
\end{array}$ & $\mathrm{K} / \mathrm{Ca}$ & $\begin{array}{l}{ }^{39} \mathrm{Ar} \\
(\%) \\
\end{array}$ & $\begin{array}{l}\text { Age } \\
(\mathrm{Ma}) \\
\end{array}$ & Std.dev. \\
\hline \multicolumn{12}{|c|}{ 1724M Lake Basalt [ $\mathrm{J}=0.00039461]$} \\
\hline \multicolumn{12}{|c|}{ plateau age $=126 \pm 14 \mathrm{ka}$; isochron age $=140 \pm 49 \mathrm{ka}$; inverse age $=143 \pm 39 \mathrm{ka}$; isochron intercept $=294.4 \pm 2.8$; total gas age $=149 \pm 17 \mathrm{ka}$} \\
\hline 550 & 136.84 & 381.7 & 0.4488 & $5.84 \mathrm{E}-16$ & 26.3 & 27 & 23.9 & 0.0009 & 0 & $34.67 \pm$ & 13.92 \\
\hline 600 & 15.923 & 5.661 & 0.05421 & $8.70 \mathrm{E}-17$ & 2.3 & 0.4 & 2.9 & 0.086 & 0.5 & $0.267 \pm$ & 0.581 \\
\hline 650 & 7.061 & 9.807 & 0.02567 & $6.09 \mathrm{E}-16$ & 4.1 & 0.69 & 10.7 & 0.05 & 4.2 & $0.207 \pm$ & 0.069 \\
\hline 700 & 4.489 & 10.962 & 0.017953 & $5.89 \mathrm{E}-16$ & 2.1 & 0.77 & 17.2 & 0.044 & 12.4 & $0.067 \pm$ & 0.032 \\
\hline 750 & 3.754 & 11.177 & 0.015156 & $1.57 \mathrm{E}-15$ & 5.4 & 0.79 & 20.7 & 0.043 & 15.3 & $0.146 \pm$ & 0.029 \\
\hline 800 & 4.444 & 11.784 & 0.017887 & $1.39 \mathrm{E}-15$ & 3.1 & 0.83 & 18.5 & 0.041 & 20.2 & $0.098 \pm$ & 0.029 \\
\hline 850 & 6.334 & 13.923 & 0.02414 & $2.39 \mathrm{E}-15$ & 5.7 & 0.98 & 16.2 & 0.035 & 13.1 & $0.257 \pm$ & 0.037 \\
\hline 900 & 9.896 & 17.129 & 0.03781 & $6.66 \mathrm{E}-16$ & 1.5 & 1.2 & 12.7 & 0.028 & 8.9 & $0.105 \pm$ & 0.051 \\
\hline 950 & 14.728 & 13.905 & 0.05316 & $5.11 \mathrm{E}-16$ & 1.2 & 0.98 & 7.3 & 0.035 & 5.8 & $0.124 \pm$ & 0.065 \\
\hline 1000 & 19.837 & 10.823 & 0.07003 & $1.29 \mathrm{E}-16$ & 0.2 & 0.76 & 4.3 & 0.045 & 6.2 & $0.03 \pm$ & 0.07 \\
\hline 1050 & 34.19 & 6.434 & 0.11844 & $-1.22 \mathrm{E}-15$ & -0.8 & 0.45 & 1.5 & 0.076 & 8.8 & $-0.197 \pm$ & 0.103 \\
\hline 1075 & 52.59 & 13.224 & 0.1779 & $1.99 \mathrm{E}-15$ & 2.1 & 0.93 & 2.1 & 0.037 & 3.5 & $0.804 \pm$ & 0.16 \\
\hline 1090 & 74.95 & 33.78 & 0.2561 & $1.20 \mathrm{E}-15$ & 2.8 & 2.4 & 3.7 & 0.0142 & 1.1 & $1.52 \pm$ & 0.315 \\
\hline \multicolumn{12}{|c|}{ 1797M Basalt under Giant Crater lava field $[\mathrm{J}=0.0004066]$} \\
\hline \multicolumn{12}{|c|}{ plateau age $=445 \pm 27 \mathrm{ka}$; isochron age $=444 \pm 76 \mathrm{ka}$; inverse age $=446 \pm 68 \mathrm{ka}$; isochron intercept $=295.4 \pm 1.9$; total gas age $=499 \pm 32 \mathrm{ka}$} \\
\hline 450 & 414.4 & 14.416 & 0.04681 & $2.24 \mathrm{E}-16$ & 97 & 1 & 8.7 & 0.034 & 0 & $275.6 \pm$ & 513.2 \\
\hline 500 & 30.07 & 10.694 & 0.10283 & $9.24 \mathrm{E}-16$ & 1.9 & 0.76 & 2.9 & 0.045 & 4.3 & $0.424 \pm$ & 0.164 \\
\hline 550 & 22.35 & 12.324 & 0.07744 & $3.96 \mathrm{E}-15$ & 2.2 & 0.87 & 4.5 & 0.039 & 21.3 & $0.366 \pm$ & 0.072 \\
\hline 600 & 17.311 & 13.729 & 0.0604 & $4.00 \mathrm{E}-15$ & 3.5 & 0.97 & 6.4 & 0.035 & 17.6 & $0.447 \pm$ & 0.064 \\
\hline 650 & 12.63 & 15.344 & 0.04478 & $3.88 \mathrm{E}-15$ & 5.3 & 1.1 & 9.6 & 0.032 & 15.4 & $0.497 \pm$ & 0.06 \\
\hline 700 & 10.773 & 15.502 & 0.03877 & $3.07 E-15$ & 5.6 & 1.1 & 11.2 & 0.031 & 13.5 & $0.447 \pm$ & 0.062 \\
\hline 750 & 11.914 & 14.064 & 0.04272 & $1.85 \mathrm{E}-15$ & 3.8 & 0.99 & 9.3 & 0.034 & 10.7 & $0.34 \pm$ & 0.071 \\
\hline 800 & 15 & 14.046 & 0.05187 & $1.83 \mathrm{E}-15$ & 5.6 & 0.99 & 7.6 & 0.035 & 5.8 & $0.621 \pm$ & 0.116 \\
\hline 850 & 20.26 & 16.065 & 0.07062 & 1.34E-15 & 3.6 & 1.1 & 6.4 & 0.03 & 4.9 & $0.538 \pm$ & 0.139 \\
\hline 900 & 42.22 & 41.78 & 0.15193 & $9.46 \mathrm{E}-16$ & 1.9 & 2.9 & 7.7 & 0.0114 & 3.1 & $0.603 \pm$ & 0.244 \\
\hline 950 & 28.59 & 41.73 & 0.10487 & $2.88 \mathrm{E}-16$ & 3.7 & 2.9 & 11.2 & 0.0114 & 0.7 & $0.805 \pm$ & 0.573 \\
\hline 951 & 30.21 & 47.5 & 0.10893 & $2.96 \mathrm{E}-16$ & 6.5 & 3.4 & 12.3 & 0.01 & 0.4 & $1.494 \pm$ & 1.58 \\
\hline 1000 & 41.58 & 98.21 & 0.15809 & $7.66 \mathrm{E}-16$ & 7.3 & 6.9 & 17.5 & 0.0046 & 0.6 & $2.37 \pm$ & 1 \\
\hline 1100 & 40.2 & 174.83 & 0.17827 & $6.69 \mathrm{E}-16$ & 5.1 & 12.3 & 27.6 & 0.0025 & 0.8 & $1.708 \pm$ & 0.902 \\
\hline 1200 & 23.69 & 148.47 & 0.11124 & 1.34E-15 & 13.3 & 10.5 & 37.5 & 0.003 & 1 & $2.57 \pm$ & 0.68 \\
\hline
\end{tabular}


TABLE 3. Cont.

\begin{tabular}{|c|c|c|c|c|c|c|c|c|c|c|c|}
\hline Temp $\left({ }^{\circ} \mathrm{C}\right)$ & ${ }^{40} \mathrm{Ar} /{ }^{39} \mathrm{Ar}$ & ${ }^{37} \mathrm{Ar} /{ }^{39} \mathrm{Ar}$ & ${ }^{36} \mathrm{Ar} /{ }^{39} \mathrm{Ar}$ & Moles ${ }^{40} \mathrm{Ar}_{\mathrm{rad}}$ & $\begin{array}{r}{ }^{40} \mathbf{A r}_{\text {rad }} \\
(\%) \\
\end{array}$ & $\begin{array}{r}{ }^{39} \mathrm{Ar}_{\mathrm{Ca}} \\
(\%)\end{array}$ & $\begin{array}{c}{ }^{36} \mathrm{Ar}_{\mathrm{Ca}} \\
(\%)\end{array}$ & $\mathrm{K} / \mathrm{Ca}$ & $\begin{array}{l}{ }^{39} \mathrm{Ar} \\
(\%)\end{array}$ & $\begin{array}{l}\text { Age } \\
\text { (Ma) }\end{array}$ & Std.dev. \\
\hline \multicolumn{12}{|c|}{ 1799M Basalt of Damons Butte [ $\mathrm{J}=0.00042789]$} \\
\hline \multicolumn{12}{|c|}{ plateau age $=144 \pm 15 \mathrm{ka}$; isochron age $=149 \pm 27 \mathrm{ka}$; inverse age $=155 \pm 23 \mathrm{ka}$; isochron intercept $=294.7 \pm 2.7$; total gas age $=153 \pm 19 \mathrm{ka}$} \\
\hline 500 & 75.26 & 34.57 & 0.2302 & 8.57E-16 & 13.4 & 2.4 & 4.2 & 0.0138 & 0.1 & $7.98 \pm$ & 3.12 \\
\hline 550 & 5.841 & 9.793 & 0.02074 & $1.13 \mathrm{E}-15$ & 9 & 0.69 & 13.3 & 0.05 & 2.9 & $0.408 \pm$ & 0.117 \\
\hline 600 & 2.804 & 11.685 & 0.01204 & $1.85 \mathrm{E}-15$ & 7.7 & 0.82 & 27.3 & 0.042 & 11.7 & $0.169 \pm$ & 0.038 \\
\hline 650 & 2.682 & 13.537 & 0.012637 & $9.07 \mathrm{E}-16$ & 2.7 & 0.96 & 30.1 & 0.036 & 17.3 & $0.056 \pm$ & 0.035 \\
\hline 700 & 3.306 & 10.721 & 0.013565 & $2.05 E-15$ & 5.7 & 0.76 & 22.2 & 0.045 & 15 & $0.146 \pm$ & 0.033 \\
\hline 750 & 3.554 & 8.473 & 0.013354 & $2.47 \mathrm{E}-15$ & 8.8 & 0.6 & 17.8 & 0.057 & 10.9 & $0.241 \pm$ & 0.037 \\
\hline 800 & 5.126 & 7.769 & 0.01885 & $1.09 \mathrm{E}-15$ & 3.9 & 0.55 & 11.6 & 0.063 & 7.5 & $0.156 \pm$ & 0.05 \\
\hline 850 & 7.011 & 9.208 & 0.02589 & $4.54 \mathrm{E}-16$ & 1.8 & 0.65 & 10 & 0.053 & 4.9 & $0.098 \pm$ & 0.073 \\
\hline 900 & 9.135 & 8.379 & 0.03272 & $5.49 \mathrm{E}-16$ & 1.8 & 0.59 & 7.2 & 0.058 & 4.7 & $0.125 \pm$ & 0.079 \\
\hline 950 & 10.471 & 9.359 & 0.03694 & $9.72 \mathrm{E}-16$ & 3.2 & 0.66 & 7.1 & 0.052 & 4 & $0.259 \pm$ & 0.091 \\
\hline 1000 & 25.51 & 11.966 & 0.08937 & $3.78 \mathrm{E}-16$ & 0.4 & 0.84 & 3.8 & 0.041 & 5.4 & $0.074 \pm$ & 0.098 \\
\hline 1050 & 10.896 & 14.523 & 0.04059 & 4.34E-16 & 1 & 1 & 10.1 & 0.033 & 5.5 & $0.084 \pm$ & 0.075 \\
\hline 1100 & 19.832 & 38.62 & 0.07781 & $1.91 \mathrm{E}-16$ & 0.2 & 2.7 & 13.9 & 0.0123 & 5.6 & $0.036 \pm$ & 0.116 \\
\hline 1150 & 24.8 & 90.52 & 0.10837 & $9.36 \mathrm{E}-16$ & 1.2 & 6.4 & 23.5 & 0.0051 & 4.2 & $0.24 \pm$ & 0.226 \\
\hline 1200 & 17.293 & 73.7 & 0.0786 & $5.10 \mathrm{E}-17$ & 1.1 & 5.2 & 26.3 & 0.0063 & 0.4 & $0.152 \pm$ & 0.963 \\
\hline
\end{tabular}

\section{M Basalt of Yellowjacket Butte [ $\mathrm{J}=\mathbf{0 . 0 0 0 4 1 8 5 3 ]}$}

plateau age $=86 \pm 14 \mathrm{ka}$; isochron age $=76 \pm 21 \mathrm{ka}$; inverse age=73 $\pm 31 \mathrm{ka}$; isochron intercept=296.2 \pm 1.8 ; total gas age=192 $\pm 18 \mathrm{ka}$

\begin{tabular}{|c|c|c|c|c|c|c|c|c|c|c|c|}
\hline 500 & 169.27 & 2.516 & 0.001951 & $1.24 \mathrm{E}-16$ & 99.8 & 0.18 & 36.2 & 0.194 & 0 & $123.43 \pm$ & 237.8 \\
\hline 550 & 15.756 & 0.05835 & 0.05262 & 6.66E-18 & 1.3 & 0.004 & 0.031 & 8.4 & 0.1 & $0.159 \pm$ & 4.31 \\
\hline 600 & 10.185 & 15.042 & 0.03473 & $2.73 \mathrm{E}-16$ & 11.5 & 1.1 & 12.2 & 0.032 & 0.6 & $0.893 \pm$ & 0.592 \\
\hline 650 & 6.254 & 10.157 & 0.02311 & $4.91 \mathrm{E}-16$ & 4.3 & 0.72 & 12.3 & 0.048 & 4.5 & $0.204 \pm$ & 0.079 \\
\hline 700 & 3.824 & 9.164 & 0.014926 & $9.22 \mathrm{E}-16$ & 4.6 & 0.65 & 17.3 & 0.053 & 13.1 & $0.132 \pm$ & 0.034 \\
\hline 750 & 3.298 & 9.17 & 0.013163 & $1.23 \mathrm{E}-15$ & 5.2 & 0.65 & 19.6 & 0.053 & 17.8 & $0.129 \pm$ & 0.028 \\
\hline 800 & 3.567 & 9.829 & 0.014711 & $3.50 \mathrm{E}-16$ & 1 & 0.69 & 18.8 & 0.05 & 24.1 & $0.027 \pm$ & 0.026 \\
\hline 850 & 4.876 & 11.106 & 0.019441 & $3.49 \mathrm{E}-16$ & 1.1 & 0.78 & 16.1 & 0.044 & 16 & $0.041 \pm$ & 0.034 \\
\hline 900 & 8.419 & 13.202 & 0.03174 & $5.30 \mathrm{E}-16$ & 1.6 & 0.93 & 11.7 & 0.037 & 9.6 & $0.104 \pm$ & 0.05 \\
\hline 950 & 14.911 & 13.468 & 0.054 & $1.56 \mathrm{E}-16$ & 0.5 & 0.95 & 7 & 0.036 & 5.3 & $0.055 \pm$ & 0.081 \\
\hline 1000 & 25.39 & 12.473 & 0.0885 & 4.13E-16 & 1.1 & 0.88 & 4 & 0.039 & 3.7 & $0.211 \pm$ & 0.118 \\
\hline 1050 & 32.61 & 13.749 & 0.11393 & $9.53 \mathrm{E}-17$ & 0.3 & 0.97 & 3.4 & 0.035 & 2.6 & $0.067 \pm$ & 0.158 \\
\hline
\end{tabular}


TABLE 3. Cont.

\begin{tabular}{|c|c|c|c|c|c|c|c|c|c|c|c|}
\hline Temp $\left({ }^{\circ} \mathrm{C}\right)$ & ${ }^{40} \mathrm{Ar} /{ }^{39} \mathrm{Ar}$ & ${ }^{37} \mathrm{Ar} /{ }^{39} \mathrm{Ar}$ & ${ }^{36} \mathrm{Ar} /{ }^{39} \mathrm{Ar}$ & Moles ${ }^{40} \mathrm{Ar}_{\mathrm{rad}}$ & $\begin{array}{r}{ }^{40} \mathrm{Ar}_{\mathrm{rad}} \\
(\%) \\
\end{array}$ & $\begin{array}{r}{ }^{39} \mathrm{Ar}_{\mathrm{Ca}} \\
(\%) \\
\end{array}$ & $\begin{array}{c}{ }^{36} \mathrm{Ar}_{\mathrm{Ca}} \\
(\%)\end{array}$ & $\mathrm{K} / \mathrm{Ca}$ & $\begin{array}{l}{ }^{39} \mathbf{A r} \\
(\%) \\
\end{array}$ & $\begin{array}{l}\text { Age } \\
(\mathrm{Ma})\end{array}$ & Std.dev. \\
\hline 1100 & 66.51 & 57.32 & 0.2332 & $1.73 \mathrm{E}-15$ & 3.6 & 4 & 6.9 & 0.0082 & 1.7 & $1.866 \pm$ & 0.295 \\
\hline 1150 & 96.51 & 242.4 & 0.3661 & $3.58 \mathrm{E}-15$ & 8.8 & 17.1 & 18.6 & 0.0017 & 0.9 & $7.69 \pm$ & 0.77 \\
\hline
\end{tabular}

\section{M Basaltic andesite of the railroad $[\mathrm{J}=0.00042147]$}

plateau age $=251 \pm 6 \mathrm{ka}$; isochron age $=248 \pm 14 \mathrm{ka}$; inverse age $=250 \pm 14 \mathrm{ka}$; isochron intercept $=295.7 \pm 2.1$; total gas age $=278 \pm 7 \mathrm{ka}$

\begin{tabular}{|c|c|c|c|c|c|c|c|c|c|c|c|}
\hline 600 & 82.12 & 1.7064 & 0.2573 & $8.78 \mathrm{E}-17$ & 7.6 & 0.12 & 0.19 & 0.287 & 0 & $4.73 \pm$ & 9.94 \\
\hline 650 & 49.44 & 0.4435 & 0.16475 & $1.56 \mathrm{E}-16$ & 1.6 & 0.031 & 0.076 & 1.1 & 0.2 & $0.601 \pm$ & 0.687 \\
\hline 700 & 14.587 & 0.04467 & 0.04835 & $2.96 \mathrm{E}-16$ & 2.1 & 0.003 & 0.026 & 11 & 1.2 & $0.231 \pm$ & 0.14 \\
\hline 750 & 9.046 & 0.11812 & 0.0289 & $5.91 \mathrm{E}-16$ & 5.7 & 0.008 & 0.11 & 4.15 & 1.4 & $0.393 \pm$ & 0.117 \\
\hline 800 & 7.34 & 0.0974 & 0.02356 & $9.40 \mathrm{E}-16$ & 5.2 & 0.007 & 0.12 & 5.03 & 3 & $0.292 \pm$ & 0.057 \\
\hline 850 & 6.714 & 0.04599 & 0.02182 & $9.33 \mathrm{E}-16$ & 4 & 0.003 & 0.059 & 10.7 & 4.3 & $0.206 \pm$ & 0.042 \\
\hline 900 & 5.944 & 0.06656 & 0.018846 & $1.90 \mathrm{E}-15$ & 6.4 & 0.005 & 0.099 & 7.36 & 6.2 & $0.29 \pm$ & 0.031 \\
\hline 950 & 6.004 & 0.05212 & 0.01945 & $1.95 \mathrm{E}-15$ & 4.3 & 0.004 & 0.075 & 9.4 & 9.3 & $0.198 \pm$ & 0.024 \\
\hline 1000 & 2.948 & 0.05278 & 0.008558 & $3.26 \mathrm{E}-15$ & 14.4 & 0.004 & 0.17 & 9.28 & 9.6 & $0.322 \pm$ & 0.019 \\
\hline 1050 & 1.7645 & 0.06615 & 0.004898 & $2.83 E-15$ & 18.3 & 0.005 & 0.38 & 7.41 & 10.9 & $0.245 \pm$ & 0.016 \\
\hline 1100 & 1.087 & 0.05442 & 0.002564 & $3.42 \mathrm{E}-15$ & 30.7 & 0.004 & 0.6 & 9 & 12.8 & $0.254 \pm$ & 0.013 \\
\hline 1150 & 1.3402 & 0.06007 & 0.003478 & $2.85 \mathrm{E}-15$ & 23.7 & 0.004 & 0.49 & 8.16 & 11.2 & $0.241 \pm$ & 0.015 \\
\hline 1200 & 0.8838 & 0.06245 & 0.002015 & $2.67 \mathrm{E}-15$ & 33.2 & 0.004 & 0.87 & 7.85 & 11.3 & $0.223 \pm$ & 0.015 \\
\hline 1250 & 0.9486 & 0.0701 & 0.002034 & $2.31 \mathrm{E}-15$ & 37.2 & 0.005 & 0.97 & 6.99 & 8.2 & $0.269 \pm$ & 0.02 \\
\hline 1300 & 1.244 & 0.07989 & 0.002072 & $3.18 \mathrm{E}-15$ & 51.3 & 0.006 & 1.1 & 6.13 & 6.2 & $0.485 \pm$ & 0.026 \\
\hline 1035 & 1.4135 & 0.12082 & 0.002759 & $2.02 \mathrm{E}-15$ & 43 & 0.009 & 1.2 & 4.06 & 4.1 & $0.462 \pm$ & 0.04 \\
\hline
\end{tabular}


Table 4. Data for ${ }^{40} \mathrm{Ar} /{ }^{39} \mathrm{Ar}$ experiments by B. Turrin at Berkeley Geochronology Center

\begin{tabular}{|c|c|c|c|c|c|c|c|c|c|c|}
\hline \multirow{2}{*}{$\frac{L a b \#}{253 M R h y}$} & \multirow{2}{*}{$\frac{\text { Temp. }{ }^{\circ} \mathrm{C}}{\text { te of Grass }}$} & \multicolumn{2}{|c|}{${ }^{40} \mathrm{Ar}{ }^{39} \mathrm{Ar}^{38} \mathrm{Ar}^{39} \mathrm{Ar}$} & \multicolumn{2}{|c|}{${ }^{37} \mathrm{Ar} /{ }^{39} \mathrm{Ar}{ }^{36} \mathrm{Ar} /{ }^{39} \mathrm{Ar}$} & \multirow[t]{2}{*}{${ }^{40^{*}} \mathrm{Ar}^{3{ }^{39}} \mathrm{Ar}{ }^{40} \mathrm{Ar}$ Moles $\times 10^{-13}$} & \multirow[t]{2}{*}{$\%{ }^{40} \mathrm{Ar}$ Rad. } & \multicolumn{3}{|c|}{$\%{ }^{39} \mathrm{Ar}$ Age (ka) $\pm 1 \mathrm{~s}(\mathrm{ka})$} \\
\hline & & r Flat & & & & & & & & \\
\hline $6238-01 \mathrm{H}$ & 1050 & 1.5010 & 0.0136 & 0.0715 & 0.0032 & & 37.5 & 0.8 & 444.1 & 25.1 \\
\hline 6238-011 & 1100 & 0.9889 & 0.0133 & 0.0678 & 0.0016 & & 53.1 & 1.6 & 414.8 & 12.2 \\
\hline $6238-01 \mathrm{~K}$ & 1200 & 0.7103 & 0.0129 & 0.0698 & 0.0008 & & 65.7 & 3.9 & 368.6 & 5.5 \\
\hline 6238-01L & 1250 & 0.6350 & 0.0127 & 0.0684 & 0.0006 & & 73.8 & 5.6 & 369.9 & 4.0 \\
\hline $6238-01 M$ & 1300 & 0.6379 & 0.0129 & 0.0696 & 0.0005 & & 76.0 & 8.0 & 382.8 & 2.9 \\
\hline $6238-01 Q$ & 1600 & 11.3509 & 0.0195 & 0.0806 & 0.0369 & & 4.0 & 1.7 & 363.0 & 46.3 \\
\hline
\end{tabular}

Plateau age $=383 \pm 0.8 \mathrm{ka}(\mathrm{n}=5$, steps M-Q)

Total fusion age $=381.9 \pm 3.2$

${ }^{40} \mathrm{Ar} /{ }^{36} \mathrm{Ar}$ intercept $=294.7 \pm 1.6$

$\mathrm{J}=0.0004377 \pm 0.0000005$

MSWD $=0.4113916$

\section{M Basaltic andesite west of Red Cap Mountain}

$\begin{array}{lrrrrr}6201-01 \mathrm{~B} & 550 & 37.9941 & 0.0452 & 0.0606 & 0.1257 \\ 6201-01 \mathrm{C} & 600 & 29.1400 & 0.0408 & 0.0690 & 0.0974 \\ 6201-01 \mathrm{D} & 650 & 60.2197 & 0.0610 & 0.0757 & 0.2021 \\ 6201-01 \mathrm{E} & 700 & 441.9174 & 0.2984 & 0.2501 & 1.4726 \\ 6201-01 \mathrm{~F} & 750 & 55.1409 & 0.0531 & 0.2904 & 0.1810 \\ 6201-01 \mathrm{G} & 800 & 219.4769 & 0.1589 & 0.9158 & 0.7390 \\ 6201-01 \mathrm{H} & 850 & 56.7436 & 0.0503 & 1.9519 & 0.1916 \\ 6201-01 \mathrm{I} & 900 & 8.2592 & 0.0173 & 3.0211 & 0.0274 \\ 6201-01 \mathrm{~J} & 950 & 6.0514 & 0.0169 & 2.9038 & 0.0198 \\ 6201-01 \mathrm{~K} & 1000 & 16.6903 & 0.0270 & 3.2481 & 0.0559 \\ 6201-01 \mathrm{~L} & 1050 & 20.3643 & 0.0364 & 8.5743 & 0.0703 \\ 6201-01 \mathrm{M} & 1100 & 20.2633 & 0.0303 & 12.7593 & 0.0714 \\ 6201-01 \mathrm{~N} & 1150 & 28.1788 & 0.0333 & 11.6629 & 0.0966\end{array}$

$\begin{array}{rrrr}2.2 & 1.6 & 67 & 291 \\ 1.2 & 9.1 & 276 & 256 \\ 0.8 & 11.6 & 392 & 278 \\ 1.5 & 16.4 & 5319 & 3662 \\ 3.0 & 0.5 & 1314 & 587 \\ 0.5 & 6.6 & 924 & 1138 \\ 0.5 & 9.1 & 225 & 179 \\ 4.8 & 26.9 & 311 & 25 \\ 7.0 & 8.0 & 332 & 29 \\ 2.5 & 3.7 & 332 & 67 \\ 1.1 & 3.3 & 185 & 80 \\ 0.7 & 2.3 & 110 & 88 \\ 1.8 & 0.8 & 411 & 224\end{array}$


Table 4, cont.

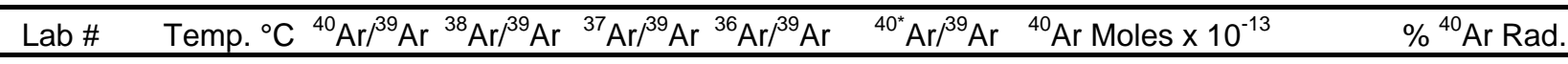

Plateau age $=309 \pm 17 \mathrm{ka}(\mathrm{n}=13$, steps B-N $)$

Total fusion age $=1180 \pm 770 \mathrm{ka}$

$\mathrm{J}=0.0004346 \pm 0.0000005$

${ }^{40} \mathrm{Ar} /{ }^{36} \mathrm{Ar}$ initial $=295.2 \pm 0.8$

$\mathrm{MSWD}=1.5$

\section{M Andesite correlated with Typhoon Mesa}

$\begin{array}{lrrrrr}6194-01 \mathrm{~A} & 550 & 66.7785 & 0.0623 & 0.0599 & 0.2240 \\ 6194-01 \mathrm{~B} & 600 & 285.7406 & 0.1915 & 0.2234 & 0.9646 \\ 6194-01 \mathrm{C} & 650 & 432.2673 & 0.2855 & 0.4121 & 1.4455 \\ 6194-01 \mathrm{D} & 700 & 57.7810 & 0.0510 & 0.2810 & 0.1953 \\ 6194-01 \mathrm{E} & 750 & 27.5406 & 0.0309 & 0.4799 & 0.0919 \\ 6194-01 \mathrm{~F} & 800 & 15.7381 & 0.0226 & 0.7855 & 0.0522 \\ 6194-01 \mathrm{G} & 850 & 3.7845 & 0.0148 & 1.2859 & 0.0119 \\ 6194-01 \mathrm{H} & 900 & 8.0928 & 0.0177 & 2.2087 & 0.0263 \\ 6194-01 \mathrm{I} & 950 & 21.9355 & 0.0278 & 3.5144 & 0.0710 \\ 6194-01 \mathrm{~J} & 1000 & 19.2512 & 0.0268 & 2.3223 & 0.0617 \\ 6194-01 \mathrm{~K} & 1050 & 12.9106 & 0.0261 & 3.1511 & 0.0420 \\ 6194-01 \mathrm{~L} & 1100 & 14.2195 & 0.0260 & 6.0306 & 0.0466 \\ 6194-01 \mathrm{M} & 1150 & 29.2751 & 0.0341 & 8.5542 & 0.0956 \\ 6194-01 \mathrm{~N} & 1200 & 47.4781 & 0.0462 & 8.6766 & 0.1537\end{array}$

$\begin{array}{rrrr}0.9 & 0.9 & 458 & 314 \\ 0.3 & 2.6 & 572 & 2097 \\ 1.2 & 7.9 & 4056 & 4179 \\ 0.2 & 9.5 & 78 & 293 \\ 1.5 & 16.1 & 337 & 96 \\ 2.3 & 22.2 & 285 & 41 \\ 9.2 & 24.2 & 277 & 12 \\ 6.0 & 7.4 & 384 & 57 \\ 5.5 & 1.9 & 958 & 133 \\ 6.3 & 2.3 & 953 & 85 \\ 5.8 & 3.0 & 595 & 56 \\ 6.5 & 1.5 & 727 & 84 \\ 5.7 & 0.4 & 1319 & 290 \\ 5.8 & 0.2 & 2168 & 638\end{array}$

Plateau age $=282 \pm 11(n=8$, steps $A-H)$

Total fusion age $=640 \pm 460$

$\mathrm{J}=0.0004378 \pm 0.0000005$

${ }^{40} \mathrm{Ar} /{ }^{36} \mathrm{Ar}$ initial $=296.1 \pm 0.8$

$\mathrm{MSWD}=0.8$

1013M Andesite of the south rim

$\begin{array}{llrlll}6199-01 \mathrm{~A} & 550 & 55.4466 & 0.0547 & 0.1317 & 0.1839 \\ 6199-01 \mathrm{~B} & 600 & 42.5197 & 0.0485 & 0.0689 & 0.1411 \\ 6199-01 \mathrm{C} & 650 & 134.6919 & 0.1111 & 0.1540 & 0.4539 \\ 6199-01 \mathrm{D} & 700 & 384.1797 & 0.2704 & 0.4655 & 1.2993 \\ 6199-01 \mathrm{E} & 750 & 245.8819 & 0.1902 & 0.5644 & 0.8289 \\ 6199-01 \mathrm{~F} & 800 & 162.3507 & 0.1360 & 0.6639 & 0.5484\end{array}$

$\begin{array}{rrrr}2.0 & 0.1 & 864 & 875 \\ 1.9 & 2.5 & 647 & 176 \\ 0.4 & 2.2 & 457 & 1438 \\ 0.1 & 5.0 & 217 & 7718 \\ 0.4 & 1.3 & 779 & 3671 \\ 0.2 & 1.4 & 283 & 2131\end{array}$


Table 4, cont.

\begin{tabular}{|c|c|c|c|c|c|c|c|c|c|c|}
\hline Lab \# & Temp. ${ }^{\circ} \mathrm{C}$ & ${ }^{40} \mathrm{Ar} /{ }^{39} \mathrm{Ar}$ & ${ }^{8} \mathrm{Ar} /{ }^{39} \mathrm{Ar}$ & ${ }^{37} \mathrm{Ar} /{ }^{39} \mathrm{Ar}$ & $\mathrm{Ar} /{ }^{39} \mathrm{Ar}$ & ${ }^{40^{*}} \mathrm{Ar} /{ }^{39} \mathrm{Ar}{ }^{40} \mathrm{Ar}$ Moles $\times 10^{-13}$ & $\%{ }^{40} \mathrm{Ar}$ Rad. & \multicolumn{3}{|c|}{$\%{ }^{39} \mathrm{Ar}$ Age $(\mathrm{ka}) \pm 1 \mathrm{~s}(\mathrm{ka})$} \\
\hline 6199-01G & 850 & 31.5990 & 0.0375 & 1.2445 & 0.1071 & & 0.1 & 5.8 & 28 & 205 \\
\hline 6199-011 & 950 & 0.5673 & 0.0111 & 0.6439 & 0.0015 & & 28.0 & 27.4 & 125 & 4 \\
\hline 6199-01J & 1000 & 0.9232 & 0.0119 & 0.7873 & 0.0028 & & 17.9 & 11.9 & 130 & 8 \\
\hline 6199-01L & 1100 & 3.5614 & 0.0178 & 3.7427 & 0.0125 & & 4.0 & 5.2 & 114 & 20 \\
\hline 6199-01M & 1150 & 6.7190 & 0.0185 & 3.9087 & 0.0229 & & 3.6 & 1.0 & 191 & 84 \\
\hline $6199-01 \mathrm{~N}$ & 1200 & 16.6357 & 0.0256 & 3.9329 & 0.0564 & & 1.7 & 0.4 & 218 & 203 \\
\hline
\end{tabular}

Plateau age $=124 \pm 3 \mathrm{ka}(\mathrm{n}=12$, steps C-N $)$

Total fusion age $=160 \pm 520$

$\mathrm{J}=0.0004361 \pm 0.0000005$

${ }^{40} \mathrm{Ar} /{ }^{36} \mathrm{Ar}$ initial $=295.0 \pm 1.1$

$\mathrm{MSWD}=0.3$

\section{M Andesite east of Grasshopper Flat}

$\begin{array}{lrrrr}6208-01 \mathrm{~F} & 770 & 19.9176 & 1.4495 & 0.0674 \\ 6208-01 \mathrm{G} & 800 & 19.7885 & 0.6354 & 0.0634 \\ 6208-01 \mathrm{H} & 850 & 16.4785 & 1.2659 & 0.0570 \\ 6208-01 \mathrm{I} & 900 & 16.5437 & 1.3033 & 0.0552 \\ 6208-01 \mathrm{~J} & 950 & 17.4649 & 2.1864 & 0.0588 \\ 6208-01 \mathrm{~K} & 1000 & 17.1810 & 3.6568 & 0.0578 \\ 6208-01 \mathrm{~L} & 1050 & 10.5151 & 6.1674 & 0.0361 \\ 6208-01 \mathrm{M} & 1100 & 8.8932 & 18.5208 & 0.0339 \\ 6208-01 \mathrm{~N} & 1150 & 16.8679 & 24.6198 & 0.0571\end{array}$

$\begin{array}{rrrr}0.5 & 0.8 & 84 & 659 \\ 5.6 & 1.1 & 870 & 486 \\ -1.6 & 2.1 & -210 & 240 \\ 2.0 & 2.5 & 262 & 209 \\ 1.5 & 2.0 & 200 & 254 \\ 2.2 & 8.0 & 296 & 143 \\ 3.0 & 63.9 & 253 & 45 \\ 3.1 & 18.4 & 218 & 56 \\ 11.0 & 1.2 & 1487 & 423\end{array}$

Plateau age $=236 \pm 33 \mathrm{ka}(\mathrm{n}=8$, steps F-M $)$

Total fusion age $=259 \pm 79 \mathrm{ka}$

$\mathrm{J}=0.0004367 \pm 0.000005$

${ }^{40} \mathrm{Ar} /{ }^{36} \mathrm{Ar}$ initial $=295.8 \pm 4.6$

$\mathrm{MSWD}=1.0$

\section{M Basaltic andesite under Giant Crater lava field}

$\begin{array}{llrlll}6197-01 B & 600 & 208.2181 & 0.1586 & 0.0860 & 0.7083 \\ 6197-01 C & 625 & 620.6958 & 0.4094 & 0.2005 & 2.1075 \\ 6197-01 D & 650 & 1313.5710 & 0.8042 & 0.5633 & 4.2327\end{array}$

$\begin{array}{rrrr}-0.5 & 4.1 & -833 & 1544 \\ -0.3 & 5.3 & -1600 & 6800 \\ 4.8 & 8.5 & 48139 & 26715\end{array}$


Table 4, cont.

\begin{tabular}{|c|c|c|c|c|c|c|c|c|c|c|}
\hline Lab \# & Temp. ${ }^{\circ} \mathrm{C}$ & ${ }^{40} \mathrm{Ar} /{ }^{39} \mathrm{Ar}$ & \multicolumn{3}{|c|}{${ }^{38} \mathrm{Ar} /{ }^{39} \mathrm{Ar}{ }^{37} \mathrm{Ar} /{ }^{39} \mathrm{Ar}^{36} \mathrm{Ar} /{ }^{39} \mathrm{Ar}{ }^{40^{*}} \mathrm{Ar} /{ }^{39} \mathrm{Ar}$} & \multirow{2}{*}{${ }^{40}$ Ar Moles $\times 10^{-13}$} & \multirow{2}{*}{$\begin{array}{r}\%{ }^{40} \mathrm{Ar} \text { Rad. } \\
1.5\end{array}$} & \multicolumn{3}{|c|}{$\%{ }^{39} \mathrm{Ar}$ Age (ka) $\pm 1 \mathrm{~s}(\mathrm{ka})$} \\
\hline 6197-01E & 675 & 103.1293 & 0.0836 & 0.2534 & 0.3438 & & & 2.1 & 1195 & 610 \\
\hline $6197-01 G$ & 750 & 92.2119 & 0.0774 & 0.8777 & 0.3121 & & 0.0 & 11.0 & 30 & 349 \\
\hline $6197-01 \mathrm{H}$ & 800 & 47.4476 & 0.0482 & 1.7160 & 0.1602 & & 0.5 & 18.2 & 184 & 137 \\
\hline 6197-01J & 900 & 9.9308 & 0.0206 & 6.6368 & 0.0346 & & 2.1 & 11.9 & 165 & 40 \\
\hline $6197-01 \mathrm{~K}$ & 950 & 20.3377 & 0.0303 & 8.5559 & 0.0703 & & 1.1 & 3.8 & 175 & 95 \\
\hline 6197-01L & 1000 & 31.0986 & 0.0435 & 8.5154 & 0.1052 & & 2.2 & 2.0 & 523 & 175 \\
\hline
\end{tabular}

Plateau age $=180 \pm 28 \mathrm{ka}(\mathrm{n}=11$, steps B-L $)$

Total fusion age $=4100 \pm 2800 \mathrm{ka}$

$\mathrm{J}=0.0004302 \pm 0.000005$

${ }^{40} \mathrm{Ar}{ }^{36} \mathrm{Ar}$ initial $=296.3 \pm 0.8$

MSWD $=1.1$

\section{MLV-004-92 Rhyolite near Cougar Butte}

6809-01A

6809-01B

11.5345

6809-01C

6.194462

6809-01D

5.590806

6809-01E

6809-01F

6809-01G

$6809-01 \mathrm{H}$

6809-01

$6809-01 \mathrm{~J}$

5.392256

5.328634

5.39254

5.50263

5.543793

5.65867

6809-01K

5.852455

6.880608

Plateau age $=437.1 \pm 6.7 \mathrm{ka}(\mathrm{n}=7$, steps $A-G)$

Total fusion age $=471 \pm 16 \mathrm{ka}$

${ }^{40} \mathrm{Ar}{ }^{36} \mathrm{Ar}$ initial $=294.9 \pm 2.6$

$\mathrm{J}=$

$\mathrm{MSWD}=0.8$

$\begin{array}{rr}0.2662721 & 3.66 \mathrm{E}-02 \\ 0.3223609 & 1.85 \mathrm{E}-02 \\ 0.5225238 & 1.66 \mathrm{E}-02 \\ 0.3072876 & 1.58 \mathrm{E}-02 \\ 0.1894166 & 1.56 \mathrm{E}-02 \\ 9.55 \mathrm{E}-02 & 1.57 \mathrm{E}-02 \\ 5.01 \mathrm{E}-02 & 1.60 \mathrm{E}-02 \\ 0.1324258 & 1.61 \mathrm{E}-02 \\ 0.1941475 & 1.62 \mathrm{E}-02 \\ 7.19 \mathrm{E}-02 & 1.65 \mathrm{E}-02 \\ 0.4604526 & 1.93 \mathrm{E}-02\end{array}$

0.7187855

0.7551547

0.7063128

0.7257053

0.732546

0.7447205

0.7554469

0.7966768

0.8702989

0.9621668

1.21829

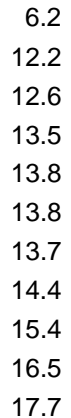

$425 \quad 43$

$446 \quad 23$

$418 \quad 21$

$429 \quad 17$

$433 \quad 15$

$440 \quad 14$

$447 \quad 14$

$471 \quad 14$

$515 \quad 18$

$569 \quad 19$ 
Table 4, cont.

\begin{tabular}{|c|c|c|c|c|}
\hline Lab \# Temp. ${ }^{\circ} \mathrm{C}{ }^{40} \mathrm{Ar} /{ }^{39} \mathrm{Ar}$ & ${ }^{38} \mathrm{Ar} /{ }^{39} \mathrm{Ar}^{37} \mathrm{Ar} /{ }^{39} \mathrm{Ar}{ }^{36} \mathrm{Ar} /{ }^{39} \mathrm{Ar}$ & ${ }^{40^{*}} \mathrm{Ar} /{ }^{39} \mathrm{Ar}$ & ${ }^{40} \mathrm{Ar}$ Moles $\times 10^{-13} \%{ }^{40} \mathrm{Ar}$ Rad. & $\%{ }^{39} \operatorname{Ar}$ Age $(\mathrm{ka}) \pm 1 \mathrm{~s}(\mathrm{ka})$ \\
\hline
\end{tabular}

\section{MLV-008-92 Lower rhyolite west of Callahan Flow (powder)}

$\begin{array}{lrrrrr}6827-01 \mathrm{~A} & 760 & 6.278712 & 1.82 \mathrm{E}-02 & 7.70 \mathrm{E}-02 & 1.88 \mathrm{E}-02 \\ 6827-01 \mathrm{~B} & 795 & 0.923601 & 1.43 \mathrm{E}-02 & 0.0782252 & 9.79 \mathrm{E}-04 \\ 6827-01 \mathrm{C} & 831 & 0.8451759 & 1.53 \mathrm{E}-02 & 8.03 \mathrm{E}-02 & 7.22 \mathrm{E}-04 \\ 6827-01 \mathrm{D} & 868 & 0.8364397 & 1.48 \mathrm{E}-02 & 7.86 \mathrm{E}-02 & 7.22 \mathrm{E}-04 \\ 6827-01 \mathrm{E} & 904 & 0.8592699 & 1.48 \mathrm{E}-02 & 7.79 \mathrm{E}-02 & 7.78 \mathrm{E}-04 \\ 6827-01 \mathrm{~F} & 940 & 0.948755 & 1.49 \mathrm{E}-02 & 7.79 \mathrm{E}-02 & 1.08 \mathrm{E}-03 \\ 6827-01 \mathrm{G} & 975 & 0.8928887 & 1.47 \mathrm{E}-02 & 7.67 \mathrm{E}-02 & 8.86 \mathrm{E}-04 \\ 6827-01 \mathrm{H} & 1011 & 0.8798807 & 1.47 \mathrm{E}-02 & 7.77 \mathrm{E}-02 & 8.26 \mathrm{E}-04 \\ 6827-01 \mathrm{I} & 1048 & 1.037567 & 1.46 \mathrm{E}-02 & 9.07 \mathrm{E}-02 & 1.29 \mathrm{E}-03 \\ 6827-01 \mathrm{~J} & 1101 & 1.576075 & 1.50 \mathrm{E}-02 & 9.23 \mathrm{E}-02 & 2.99 \mathrm{E}-03 \\ 6827-01 \mathrm{~K} & 1190 & 1.398656 & 1.47 \mathrm{E}-02 & 8.60 \mathrm{E}-02 & 2.46 \mathrm{E}-03 \\ 6827-01 \mathrm{~L} & 1512 & 14.39404 & 2.34 \mathrm{E}-02 & 0.2379536 & 4.59 \mathrm{E}-02\end{array}$

0.712626

0.6316958

0.6294202

0.620623

0.6266806

0.6280611

0.6284603

0.6331514

0.6535724

0.6916852

0.6692882

0.8368423

11.4
69.0
75.2
75.0
73.7
66.8
71.1
72.7
63.5
44.1
48.1
5.8

431

393

392

386

390

391

391

394

407

431

417

521

Plateau age $=391 \pm 2 \mathrm{ka}(\mathrm{n}=8$, steps $\mathrm{A}-\mathrm{H})$

Total fusion age $=$

$\mathrm{J}=0.000345 \pm 0.000005$

${ }^{40} \mathrm{Ar} /{ }^{36} \mathrm{Ar}$ initial $=$

MSWD =

\section{MLV-014-92 Lake Basalt (1724M site)}

$\begin{array}{lrrrrr}6829-01 \mathrm{C} & 766 & 181.9422 & 0.1323654 & 2.130739 & 0.6119313 \\ 6829-01 \mathrm{D} & 809 & 39.27193 & 4.18 \mathrm{E}-02 & 4.349382 & 0.1334935 \\ 6829-01 \mathrm{E} & 852 & 19.59004 & 2.78 \mathrm{E}-02 & 6.769291 & 6.72 \mathrm{E}-02 \\ 6829-01 \mathrm{~F} & 895 & 9.200212 & 1.99 \mathrm{E}-02 & 8.088644 & 3.26 \mathrm{E}-02 \\ 6829-01 \mathrm{G} & 939 & 10.2117 & 2.01 \mathrm{E}-02 & 7.578131 & 3.58 \mathrm{E}-02 \\ 6829-01 \mathrm{H} & 981 & 17.61159 & 2.53 \mathrm{E}-02 & 8.314636 & 6.10 \mathrm{E}-02 \\ 6829-01 \mathrm{I} & 1024 & 60.47002 & 5.32 \mathrm{E}-02 & 10.21348 & 0.2068575\end{array}$

Plateau age $=123 \pm 27(n=7$, steps C- 1$)$

Total fusion age $=$

$\mathrm{J}=0.000356$

${ }^{40} \mathrm{Ar}{ }^{36} \mathrm{Ar}$ initial $=$

MSWD = 
Table 4, cont.

\begin{tabular}{|c|c|c|c|c|c|c|c|c|c|c|c|}
\hline Lab \# & \multicolumn{5}{|c|}{ Temp. ${ }^{\circ} \mathrm{C}^{40} \mathrm{Ar} /{ }^{39} \mathrm{Ar}{ }^{38} \mathrm{Ar} /{ }^{39} \mathrm{Ar}{ }^{37} \mathrm{Ar} /{ }^{39} \mathrm{Ar}{ }^{36} \mathrm{Ar} /{ }^{39} \mathrm{Ar}$} & ${ }^{40^{*}} \mathrm{Ar} /{ }^{39} \mathrm{Ar}$ & ${ }^{40} \mathrm{Ar}$ Moles $\times 10^{-13}$ & $\%{ }^{40} \mathrm{Ar}$ Rad. & \multicolumn{3}{|c|}{$\%{ }^{39} \mathrm{Ar}$ Age $(\mathrm{ka}) \pm 1 \mathrm{~s}(\mathrm{ka})$} \\
\hline 6828-02D & 795 & 25.3708 & 0.0339 & 2.5209 & 0.0866 & & 7.33 & -0.1 & 5.2 & -19 & 111 \\
\hline $6828-02 G$ & 850 & 12.9269 & 0.0240 & 4.9887 & 0.0444 & & 1.96 & 1.4 & 2.7 & 119 & 59 \\
\hline 6828-02H & 868 & 16.5139 & 0.0254 & 5.4134 & 0.0565 & & 7.38 & 1.3 & 7.9 & 136 & 71 \\
\hline 6828-021 & 886 & 6.5813 & 0.0189 & 5.2849 & 0.0231 & & 6.36 & 2.4 & 17.0 & 103 & 29 \\
\hline 6828-02L & 940 & 4.8528 & 0.0199 & 6.5320 & 0.0178 & & 1.76 & 2.0 & 6.5 & 62 & 28 \\
\hline 6828-02M & 959 & 6.0973 & 0.0214 & 7.2551 & 0.0223 & & 1.77 & 1.0 & 5.1 & 41 & 34 \\
\hline $6828-02 \mathrm{~N}$ & 977 & 7.7178 & 0.0228 & 7.8263 & 0.0283 & & 1.72 & -0.7 & 3.9 & -33 & 38 \\
\hline $6828-020$ & 993 & 10.8424 & 0.0260 & 8.2910 & 0.0380 & & 2.01 & 2.2 & 3.2 & 157 & 56 \\
\hline 6828-02P & 1010 & 14.8345 & 0.0286 & 7.8719 & 0.0526 & & 2.35 & -0.7 & 2.8 & -66 & 75 \\
\hline $6828-02 Q$ & 1046 & 19.3874 & 0.0325 & 5.6111 & 0.0677 & & 6.13 & -0.9 & 5.6 & -118 & -86 \\
\hline $6828-02 R$ & 1083 & 25.4060 & 0.0367 & 5.4786 & 0.0878 & & 7.12 & -0.4 & 4.9 & -74 & -115 \\
\hline 6828-02S & 1154 & 72.9348 & 0.0693 & 19.3980 & 0.2503 & & 3.62 & 0.6 & 0.9 & -286 & -345 \\
\hline
\end{tabular}

Plateau age $=65 \pm 10 \mathrm{ka}(\mathrm{n}=10$, steps $D-M)$

Total fusion age $=41 \pm 19 \mathrm{ka}$

$\mathrm{J}=0.000356$

${ }^{40} \mathrm{Ar} /{ }^{36} \mathrm{Ar}$ initial $=294.6 \pm 0.8$

$\mathrm{MSWD}=1.8$

\section{MLV-017-92 Rhyolite of Mount Hoffman}

\begin{tabular}{|c|c|c|c|c|c|}
\hline $6820-01 \mathrm{~A}$ & 725 & 5.694029 & $1.89 \mathrm{E}-02$ & 0.2079746 & 1.90E-02 7.43E-02 \\
\hline 6820-01B & 760 & 2.139158 & $1.65 \mathrm{E}-02$ & 0.251862 & 7.17E-03 3.18E-02 \\
\hline $6820-01 C$ & 796 & 2.151766 & 1.67E-02 & 0.2626261 & 7.19E-03 3.76E-02 \\
\hline 6820-01D & 831 & 2.179277 & 1.67E-02 & 0.2473247 & 7.27E-03 4.12E-02 \\
\hline 6820-01E & 868 & 2.202302 & $1.65 \mathrm{E}-02$ & 0.2712605 & 7.26E-03 6.96E-02 \\
\hline $6820-01 G$ & 975 & 2.874001 & 1.75E-02 & 0.9468883 & $9.16 \mathrm{E}-030.2310406$ \\
\hline $6820-01 \mathrm{H}$ & 975 & 2.883863 & 0.0175311 & 0.9482795 & $9.14 \mathrm{E}-030.2480674$ \\
\hline $6820-011$ & 1046 & 3.308423 & $1.79 \mathrm{E}-02$ & 1.857564 & 1.04E-02 0.3791229 \\
\hline $6820-01 \mathrm{~J}$ & 1550 & 8.428307 & $2.08 \mathrm{E}-02$ & 3.838092 & 2.66E-02 0.8458053 \\
\hline
\end{tabular}

$\begin{array}{rrr}1.3 & 46 & 23 \\ 1.5 & 20 & 9 \\ 1.8 & 23 & 9 \\ 1.9 & 25 & 10 \\ 3.2 & 43 & 10 \\ 8.1 & 143 & 28 \\ 8.6 & 154 & 20 \\ 11.5 & 235 & 30 \\ 10.0 & 523 & 44\end{array}$


Table 4, cont.

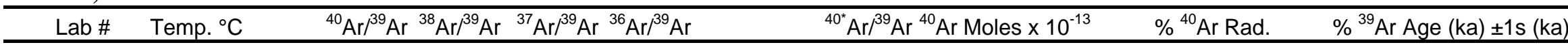

Plateau age $=28 \pm 5 \mathrm{ka}(n=5$, steps A-E $)$

Total fusion age $=$

$\mathrm{J}=0.000343 \pm 0.000005$

${ }^{40} \mathrm{Ar} /{ }^{36} \mathrm{Ar}$ initial $=$

MSWD =

\section{MLV-020-92 Basalt of Mammoth Crater}

6824-01E

$780 \quad 36.06165$

6824-01F $\quad 806 \quad 33.21315$

6824-01G $\quad 830 \quad 31.32972$

6824-01H $\quad 855 \quad 26.12564$

6824-011 $\quad 880 \quad 19.76553$

6824-01J $\quad 905 \quad 11.09913$

6824-01K $\quad 929 \quad 9.283259$

6824-01L $\quad 954 \quad 8.870646$

6824-01M $\quad 1003 \quad 8.614202$

$\begin{array}{lll}6824-01 N & 1102 & 8.988492\end{array}$

0.29611630 .12154340 .1596714

$0.2764547 \quad 0.112080 .1060781$

$0.25192420 .10584376 .36 \mathrm{E}-02$

$0.2637485 \quad 8.82 \mathrm{E}-020.0879904$

$0.2701008 \quad 6.69 \mathrm{E}-021.36 \mathrm{E}-02$

$0.2754547 \quad 3.78 \mathrm{E}-02-7.07 \mathrm{E}-02$

$0.3113599 \quad 3.15 \mathrm{E}-02-2.03 \mathrm{E}-02$

$0.3376323 \quad 3.00 \mathrm{E}-02$ 1.02E-02

$0.4098364 \quad 2.93 \mathrm{E}-02-2.44 \mathrm{E}-02$

$0.615153 \quad 3.01 \mathrm{E}-020.1310751$

Total fusion age $=36 \pm 16 \mathrm{ka}$

${ }^{40} \mathrm{Ar} /{ }^{36} \mathrm{Ar}$ initial $=295.9 \pm 0.6$

$\mathrm{J}=0.000332 \pm 0.000006$

MSWD $=7.7$

\section{MLV-021-92 Andesite of Schonchin Butte}

\section{4-02B}

66.4710

6824-02C

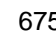

27.4216

6824-02E

700

6824-02F

$6824-02 G$

16.5867

6824-02H

6824-02

6824-02J

10.5163

$6824-02 \mathrm{~K}$

6824-02L

15.7534

11.8279

$\begin{array}{rr}6.4816 & 0.22091 .683336 \\ 1.3379 & 0.12360 .5273134 \\ 0.3814 & 9.37 \mathrm{E}-02-0.2443824 \\ 0.9117 & 7.23 \mathrm{E}-021.60 \mathrm{E}-02 \\ 0.3040 & 5.59 \mathrm{E}-02 \text { 8.62E-02 } \\ 0.6179 & 4.37 \mathrm{E}-020.1775405 \\ 1.2255 & 3.56 \mathrm{E}-020.1263156 \\ 2.2048 & 3.57 \mathrm{E}-020.1176284 \\ 1.4915 & 4.31 \mathrm{E}-02-0.1371585 \\ 3.0899 & 5.43 \mathrm{E}-02-5.43 \mathrm{E}-02 \\ 19.7040 & 4.49 \mathrm{E}-025.09 \mathrm{E}-02\end{array}$

2.5

1.4

$\begin{array}{rr}96 & 102 \\ 63 & 50 \\ 38 & 86 \\ 53 & 42 \\ 8 & 29 \\ -42 & 16 \\ -12 & 18 \\ 6 & 14 \\ -15 & 11 \\ 78 & 11\end{array}$


Table 4, cont.

\begin{tabular}{llllll}
\hline Lab \# & Temp. ${ }^{\circ} \mathrm{C}$ & ${ }^{40} \mathrm{Ar} /{ }^{39} \mathrm{Ar}{ }^{38} \mathrm{Ar} /{ }^{39} \mathrm{Ar}{ }^{37} \mathrm{Ar} /{ }^{39} \mathrm{Ar}{ }^{36} \mathrm{Ar} /{ }^{39} \mathrm{Ar}$ & ${ }^{40} \mathrm{Ar} /{ }^{39} \mathrm{Ar}{ }^{40} \mathrm{Ar}$ Moles $\times 10^{-13}$ & $\%{ }^{40} \mathrm{Ar} \operatorname{Rad}$. & $\%{ }^{39} \mathrm{Ar} \mathrm{Age}(\mathrm{ka}) \pm 1 \mathrm{~s}(\mathrm{ka})$ \\
\hline
\end{tabular}

Plateau age $=65 \pm 23 \mathrm{ka}(\mathrm{n}=11$, steps B-L $)$

Total fusion age $=63 \pm 93 \mathrm{ka}$

${ }^{40} \mathrm{Ar} /{ }^{36} \mathrm{Ar}$ initial $=295.7 \pm 1.3$

$\mathrm{J}=$

$\mathrm{MSWD}=2.9$

\section{4-27-94 Basalt of Tionesta (data from Turrin, 1996)}

$\begin{array}{lrrrrr}8195-01 \mathrm{~A} & 700 & 359.4786 & 0.2481 & 9.3548 & 1.1750 \\ 8195-01 \mathrm{~B} & 750 & 240.4905 & 0.1578 & 13.2766 & 0.7804 \\ 8195-01 \mathrm{C} & 775 & 196.6317 & 0.1397 & 17.6941 & 0.6330 \\ 8195-01 \mathrm{D} & 800 & 141.5450 & 0.0978 & 23.2794 & 0.4483 \\ 8195-01 \mathrm{E} & 825 & 104.2381 & 0.0771 & 25.6951 & 0.3364 \\ 8195-01 \mathrm{~F} & 850 & 91.2698 & 0.0727 & 22.1938 & 0.2997 \\ 8195-01 \mathrm{G} & 875 & 82.5349 & 0.0635 & 21.5624 & 0.2687 \\ 8195-01 \mathrm{H} & 901 & 67.0430 & 0.0540 & 23.2465 & 0.2190 \\ 8195-01 \mathrm{I} & 950 & 66.6362 & 0.0522 & 19.0183 & 0.2167 \\ 8195-01 \mathrm{~J} & 1000 & 60.0434 & 0.0491 & 15.6397 & 0.1961 \\ 8195-01 \mathrm{~K} & 1100 & 75.3622 & 0.0579 & 13.9246 & 0.2466 \\ 8195-01 \mathrm{~L} & 1200 & 123.3804 & 0.0870 & 16.0252 & 0.4011\end{array}$

$\begin{array}{rrrrr}0.535 & 3.6 & 1.6 & 3005 & 1874 \\ 0.480 & 4.6 & 2.2 & 2539 & 993 \\ 0.396 & 5.6 & 2.2 & 2557 & 728 \\ 0.558 & 7.7 & 4.2 & 2553 & 410 \\ 0.455 & 6.6 & 4.6 & 1611 & 280 \\ 0.422 & 4.9 & 4.9 & 1046 & 234 \\ 0.455 & 5.9 & 5.8 & 1132 & 196 \\ 0.421 & 6.2 & 6.7 & 979 & 151 \\ 0.726 & 6.2 & 11.6 & 962 & 109 \\ 0.944 & 5.6 & 16.8 & 777 & 81 \\ 1.56 & 4.8 & 22.1 & 836 & 86 \\ 2.01 & 5.0 & 17.4 & 1427 & 198\end{array}$

Inverse isochron age is preferred: $54 \pm 16 \mathrm{ka}$

Plateau age $896 \pm 56 \mathrm{ka}(\mathrm{n}=6$, steps F-K)

Total fusion age $=1200 \pm 210 \mathrm{ka}$

$\mathrm{J}=0.0001272$

${ }^{40} \mathrm{Ar}{ }^{36} \mathrm{Ar}$ initial $=311.8 \pm 2.8$

$\mathrm{MSWD}=1.1$ 
Table 5. Comparison of early rhyolite ages, Medicine Lake volcano

Ages in ka (thousands of years) except as noted

\begin{tabular}{|c|c|c|c|c|c|}
\hline $\begin{array}{l}\text { Rhyolite } \\
\text { Unit }\end{array}$ & K-Ar Ages & Sample \# & ${ }^{40} \mathrm{Ar} /{ }^{39} \mathrm{Ar}$ Age & Sample \# & $\begin{array}{l}\text { Previously Published } \\
\text { K-Ar Ages, in Ma }\end{array}$ \\
\hline \multirow[t]{3}{*}{$r c b$} & $547 \pm 16$ & 1359M & $437 \pm 7$ & MLV-004-92 & $0.43 \pm 0.04$ (Mertzman, 1982, no. 11$)$ \\
\hline & $574 \pm 21$ & $155 \mathrm{M}$ & & & \\
\hline & $609 \pm 23$ & $155 \mathrm{M}$ & & & \\
\hline \multirow[t]{13}{*}{ rgf } & $364 \pm 11$ & $1351 \mathrm{M}$ & $391 \pm 2$ & MLV-008-92 & $0.61 \pm 0.03$ (Mertzman, 1982, no. 10$)$ \\
\hline & $361 \pm 11$ & $1351 \mathrm{M}$ & & & \\
\hline & $486 \pm 20$ & $142 \mathrm{M}$ & & & \\
\hline & $389 \pm 12$ & $52-4-628$ & & & $0.29 \pm 0.02$ (Mertzman, 1982, no. 8) \\
\hline & $378 \pm 9$ & $52-4-628$ & & & $0.33 \pm 0.02($ Mertzman, 1982, no. 12$)$ \\
\hline & $251 \pm 10$ & $1356 \mathrm{M}$ & & & \\
\hline & $351 \pm 11$ & $1162 \mathrm{M}$ & & & \\
\hline & $364 \pm 11$ & 1162M & & & \\
\hline & $486 \pm 43$ & $253 \mathrm{M}$ & $383 \pm 1$ & $253 M$ & \\
\hline & $430 \pm 26$ & $253 \mathrm{M}$ & & & \\
\hline & $263 \pm 8$ & $253 M$ & & & \\
\hline & $284 \pm 9$ & $253 M$ & & & \\
\hline & & & \multicolumn{3}{|c|}{$387 \pm 6=$ simple mean and one std. dev. } \\
\hline \multirow[t]{4}{*}{ rsl } & $304 \pm 9$ & $1365 \mathrm{M}$ & & & $0.24 \pm 0.03$ (Mertzman, 1982, no. 9) \\
\hline & $312 \pm 9$ & $1365 \mathrm{M}$ & & & \\
\hline & $364 \pm 18$ & $256 \mathrm{M}$ & & & \\
\hline & $302 \pm 15$ & $256 \mathrm{M}$ & & & \\
\hline \multirow[t]{2}{*}{ reg } & $607 \pm 44$ & $103 \mathrm{M}$ & $475 \pm 29$ & $1707 \mathrm{M}$ & $0.48 \pm 0.06$ (Mertzman, 1983, no. 16$)$ \\
\hline & $732 \pm 22$ & $103 \mathrm{M}$ & & & \\
\hline
\end{tabular}

